\title{
Numerical and experimental study on the ability of dynamic roughness to alter the development of a leading edge vortex
}

\author{
Christopher D. Griffin \\ West Virginia University
}

Follow this and additional works at: https://researchrepository.wvu.edu/etd

\section{Recommended Citation}

Griffin, Christopher D., "Numerical and experimental study on the ability of dynamic roughness to alter the development of a leading edge vortex" (2013). Graduate Theses, Dissertations, and Problem Reports. 320.

https://researchrepository.wvu.edu/etd/320

This Dissertation is protected by copyright and/or related rights. It has been brought to you by the The Research Repository @ WVU with permission from the rights-holder(s). You are free to use this Dissertation in any way that is permitted by the copyright and related rights legislation that applies to your use. For other uses you must obtain permission from the rights-holder(s) directly, unless additional rights are indicated by a Creative Commons license in the record and/ or on the work itself. This Dissertation has been accepted for inclusion in WVU Graduate Theses, Dissertations, and Problem Reports collection by an authorized administrator of The Research Repository @ WVU.

For more information, please contact researchrepository@mail.wvu.edu. 


\author{
Christopher D. Griffin
}

Dissertation submitted to the

Benjamin M. Statler College of Engineering and Mineral Resources

at West Virginia University

in partial fulfillment of the requirements for the degree of

Doctor of Philosophy
in
Aerospace Engineering

Wade W. Huebsch, Ph.D., Chair

Darran R. Cairns, Ph.D.

Ismail Celik, Ph.D.

John M. Kuhlman, Ph.D.

Alric P. Rothmayer, Ph.D.

Department of Mechanical and Aerospace Engineering

Morgantown, West Virginia

2013

Copyright 2013 Christopher D. Griffin 


\section{ABSTRACT \\ Numerical and Experimental Study on the Ability of Dynamic Roughness to Alter the
Development of a Leading Edge Vortex}

Christopher Griffin

Dynamic stall is an unsteady aerodynamic phenomenon garnering much research interest because it occurs in a variety of applications. For example, dynamic stall is known to occur on helicopter rotor blades, wind turbines, high maneuvering military aircraft, and flapping wings. Dynamic stall occurs when an aerodynamic lifting device, such as an airfoil, wing, or turbomachine blade, undergoes a rapid pitching motion. It also occurs on lifting devices that are impulsively started at high angles of attack. Dynamic stall can "delay" aerodynamic stall to angles of attack that are significantly beyond the static stall angle of attack.

During dynamic stall a large leading edge vortex (LEV) is formed, which creates greater fluid acceleration over the wing or airfoil, thus sustaining lift. As this vortex is shed downstream stall eventually occurs and there is an abrupt increase in drag and a large shift in pitching moment. Research has been performed to better understand the mechanisms occurring during dynamic stall in an effort to find ways to best take advantage of the increased lift associated with dynamic stall, but avoid the downfalls that occur once stall is initiated. Few attempts have been made to alter the LEV, and these attempts have used methods associated with laminar boundary layer separation control. Although these methods have shown promise, they suffer from the drawback that they exhaust more energy than is gained by flow control, while also only being effective at certain flight regimes.

The research described herein documents the first study on the ability of dynamic roughness to alter the LEV encountered on a rapidly pitching airfoil. Both numerical and experimental studies were performed, including two-dimensional and three-dimensional computational fluid dynamics (CFD) simulations as well as stereo and planar particle image velocimetry (PIV) experiments. Evidence for the ability of small scale dynamic roughness to alter the development of the LEV was found in both the computational simulations and experiments. This research is the first of its kind to show both computationally and experimentally that dynamic roughness is a viable flow control method for both steady and unsteady aerodynamics. 


\section{TABLE OF CONTENTS}

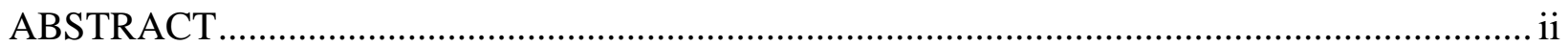

TABLE OF CONTENTS ................................................................................................ ii

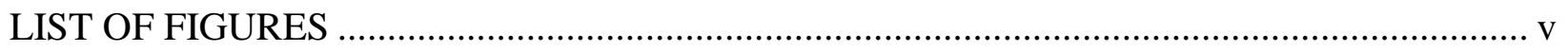

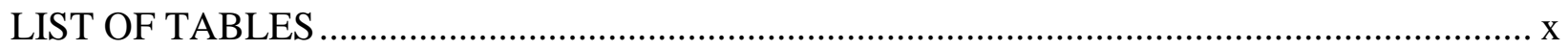

CHAPTER 1: $\quad$ INTRODUCTION ............................................................................... 1

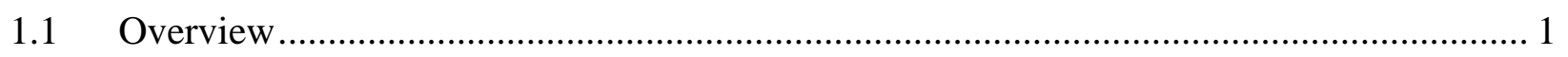

1.2 Dynamic Roughness at Static Angle of Attack...................................................... 3

1.3 Previous Numerical Analysis of Dynamic Stall ................................................... 5

1.4 Previous Experimental Analysis of Dynamic Stall................................................. 9

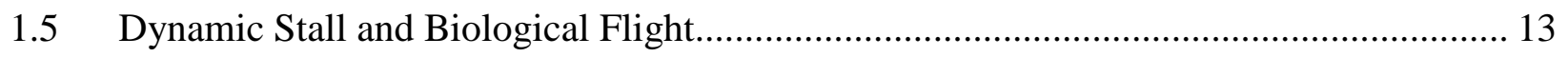

1.6 Previous Research Concerning the Alteration of the Leading Edge Vortex .................... 15

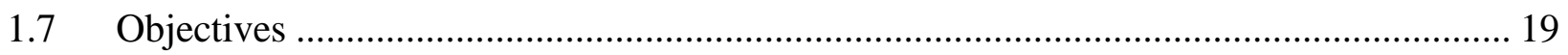

CHAPTER 2: $\quad$ EXPERIMENTAL ANALYSIS .......................................................... 20

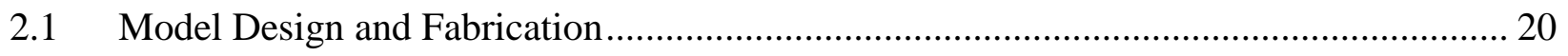

2.2 Pitching Mechanism and Dynamic Roughness Actuation System ................................. 23

2.3 Instrumentation and Experimental Setup............................................................. 26

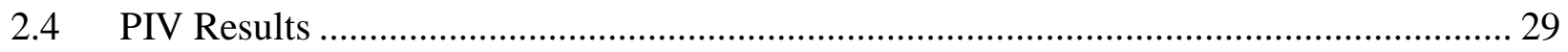

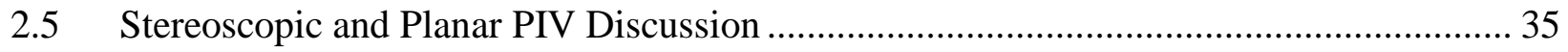

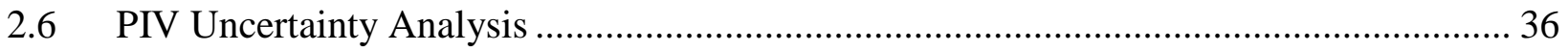

CHAPTER 3: $\quad$ COMPUTATIONAL FLUID DYNAMICS .............................................. 41

3.1 Computational Mesh and Solver Considerations................................................... 41

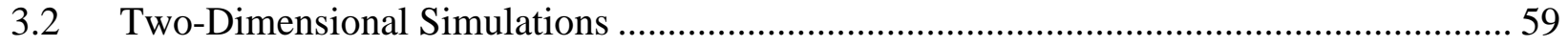

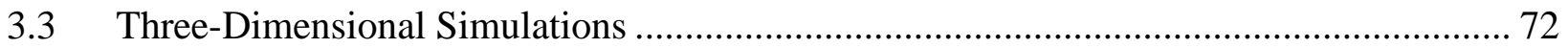




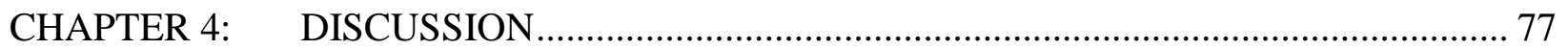

CHAPTER 5: $\quad$ CONCLUSIONS AND RECOMMENDATIONS ...................................... 81

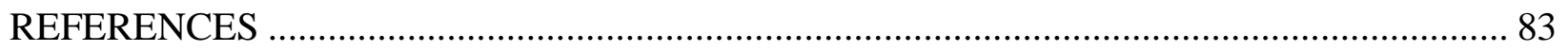

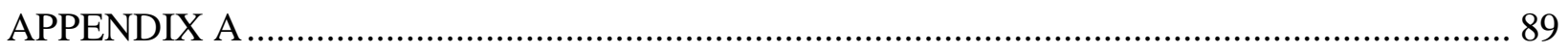

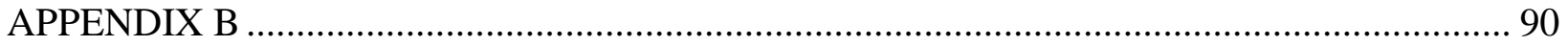

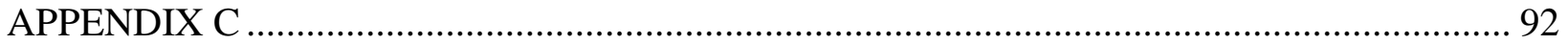

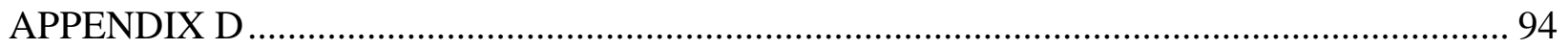




\section{LIST OF FIGURES}

Figure 1.1 Events of dynamic stall on NACA 0012 airfoil [2] .......................................... 2

Figure 1.2 Surface pressure contour and pathline plots of a clean airfoil and an airfoil with dynamic roughness ridges, respectively, at $12^{\circ}$ angle of attack and 100,000 Reynolds number [5]......................................................................... 5

Figure 1.3 Smoke visualization of a NACA 0012 airfoil at $9.5^{\circ}$ angle of attack and 100,000 Reynolds number with no dynamic roughness and with dynamic roughness active [5]. 5

Figure 1.4 Evolution of vorticity field: 1) leading-edge vortex, 2) shear layer vortex, 3)

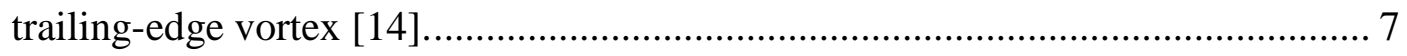

Figure 1.5 Classification of critical points and corresponding location in the computed flow field [17]. 8

Figure 1.6 Flow visualization for $R e_{\mathrm{c}}=45,000$ and constant pitching rate with a nondimensional pitching rate of $0.2(\mathrm{a})$ and $0.6(\mathrm{~b})$ [27]. 11

Figure 1.7 Comparison of computational work done by Liu, et al. (a) [36], with smoke visualization done by Ellington, et al. (b) [35].

Figure 1.8 Smoke flow visualization from Freymuth, et al. [44] showing the effect of flow control on a pitching airfoil using a rotating cylinder leading edge. Both airfoils are at $40^{\circ}$ during their pitching evolution, without nose rotation (a) and with nose rotation (b). 16

Figure 1.9 Computational results by Visbal [45] of a pitching airfoil at $36^{\circ}$ in its pitching evolution with (a) no control techniques, (b) distributed surface suction, and (c) a moving wall surface. 16

Figure 1.10 Variation of suction flow rate for complete suppression of the LEV with angle of attack, for different pitch rates [46]. 17

Figure 1.11 Results from work done by Heubsch [9] showing the LEV on the leading edge of a clean parabola (a) and the effect of dynamic roughness (b), all at the same physical time step and angle of attack. 18 


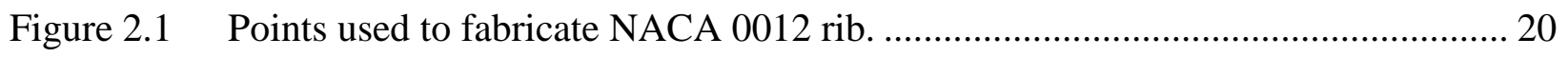

Figure 2.2 CAD representation of aluminum rib for NACA 0012 airfoil.................................. 21

Figure 2.3 Exploded view and assembled view of dynamic roughness section (CAD representation), respectively.......................................................................... 21

Figure 2.4 Assembly of NACA 0012 model with aluminum sheet metal skin removed......... 22

Figure 2.5 Mechanism designed and developed to oscillate an airfoil. ..................................... 23

Figure 2.6 Dynamic roughness pneumatic actuation system................................................ 25

Figure 2.7 Labeled image of PIV setup, highlighting positioning of cameras and laser output with respect of wind tunnel test section. .................................................................. 27

Figure 2.8 Mounting position of the NACA 0012 model in the WVU subsonic closed circuit

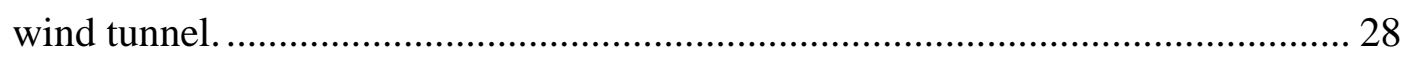

Figure 2.9 Planar PIV results of the evolution of the LEV at varying Reynolds numbers at the

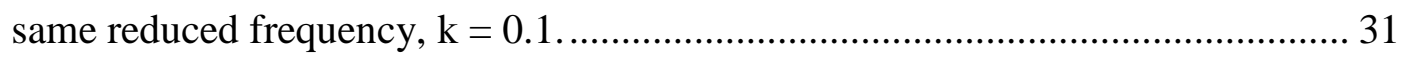

Figure 2.10 Planar PIV results of the change in LEV formation as reduced frequency, k, is

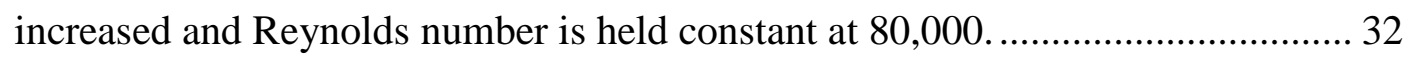

Figure 2.11 Contours of chord-wise velocity magnitude overlaid with velocity vectors and streamlines at $\operatorname{Re}=150,000$ and $k=0.1$. At this flow condition dynamic roughness was able to delay the development of the LEV ...................................... 33

Figure 2.12 Contours of chord-wise velocity magnitude overlaid with velocity vectors and streamlines at $\operatorname{Re}=80,000$ and $\mathrm{k}=0.1$. At this flow condition dynamic roughness did not have any noticeable effect on LEV development. 34

Figure 2.13 Chord-wise velocity contours with velocity vectors for the same flow condition with collected with (a) planar PIV and (b) stereoscopic PIV................................... 35

Figure 2.14 Contours of span-wise velocity from stereoscopic PIV analysis. ........................... 36

Figure 3.1 Nodes do not have to be aligned along a mesh interface using the sliding mesh technique. ............................................................................................................. 42 
Figure 3.2 Mesh used for dynamic stall simulation. Allows for use of three different methods simulating a pitching airfoil.

Figure 3.3 View of mesh highlighting the structured zone within unstructured zone as well as the Dynamic Roughness Zone above the roughness elements on the airfoil surface.

Figure 3.4 Comparison between streamline results provided by the (a) dynamic mesh method and the (b) sliding mesh method. 46

Figure 3.5 Computed airfoil surface pressure distribution for the dynamic mesh method and sliding mesh method when airfoil is at $22.5^{\circ}$ angle of attack. 46

Figure 3.6 Representation of how angle of attack was calculated for (a) rotating airfoil and (b) rotating boundary with stationary airfoil. 47

Figure 3.7 Two-dimensional streamlines of a rapidly pitching airfoil at $22.5^{\circ}$ angle of attack for (a) Choudhuri, et al. results; (b) airfoil pitching about 25\% chord; (c) airfoil fixed with domain rotating about $25 \%$ chord; and (d) variable velocity vector prescribed over velocity inlet. 48

Figure 3.8 Streamlines at $22.5^{\circ}$ for a (a) pitching airfoil in a stationary domain, (b) rotating domain with a fixed airfoil, and (c) variable velocity vector.

Figure 3.9 Grid refinement study observing initiation of LEV development for (a) Mesh 1, (b) Mesh 2, (c) Mesh 3, and (d) Mesh 4. 51

Figure 3.10 Grid refinement study observing LEV structure for (a) Mesh 1, (b) Mesh 2, (c) Mesh 3, and (d) Mesh 4. 52

Figure 3.11 Temporal refinement study observing initiation of LEV development for a $\Delta \mathrm{t}^{*}=$ (a) 0.01, (b) 0.005, (c) 0.001, and (d) 0.0005 . 53

Figure 3.12 Temporal refinement study observing vortex shedding for a $\Delta t^{*}=$ (a) 0.01 , (b) 0.005, (c) 0.001, and (d) 0.0005 . 53

Figure 3.13 Revised two-dimensional grid use for CFD simulations. 54

Figure 3.14 Close-up view of the near airfoil region highlighting the extents of the DR Zone.55 
Figure 3.15 Half a cycle of a two-dimensional dynamic roughness element, starting from a clean surface (a) and reaching its maximum amplitude (f) .............................. 57

Figure 3.16 Half a cycle of three-dimensional dynamic roughness elements, starting from a clean surface (a) and reaching its maximum amplitude (f) .............................. 58

Figure 3.17 Contour plot of vorticity magnitude for a pitching airfoil just before LEV development with maximum DR element amplitude to highlight the sub-boundary layer nature of the dynamic roughness.

Figure 3.18 Streamline plots colored by vorticity magnitude of the variation in LEV at different Reynolds numbers while reduced frequency is held constant. 61

Figure 3.19 Streamline plots colored by vorticity magnitude at three different reduced frequency values. 62

Figure 3.20 Streamlines colored by vorticity magnitude for an airfoil undergoing dynamic stall at $\operatorname{Re}=10,000$ without and with dynamic roughness actuation, respectively. ...... 63

Figure 3.21 Streamlines colored by vorticity magnitude for an airfoil undergoing dynamic stall at $\operatorname{Re}=80,000$ without and with dynamic roughness actuation, respectively. ...... 64

Figure 3.22 Streamlines colored by vorticity magnitude for an airfoil undergoing dynamic stall at $\operatorname{Re}=150,000$ without and with dynamic roughness actuation, respectively. .... 65

Figure 3.23 Streamlines colored by vorticity magnitude for an airfoil undergoing dynamic stall at $\mathrm{Re}=150,000$ without and with dynamic roughness actuation, respectively. The amplitude of the dynamic roughness is half as in the previous figure.

Figure 3.24 Streamlines colored by vorticity magnitude for an airfoil undergoing dynamic stall at $\operatorname{Re}=150,000$ without and with dynamic roughness actuation, respectively. Frequency of dynamic roughness actuation reduced to $30 \mathrm{~Hz}$ while amplitude is unchanged from previous results. 68

Figure 3.25 Streamlines colored by vorticity magnitude for an airfoil undergoing dynamic stall at $\operatorname{Re}=150,000$ with dynamic roughness actuation at a constant amplitude $(10 \%$ roughness diameter), but with an actuation frequency varying from $30 \mathrm{~Hz}, 60 \mathrm{~Hz}$, and $90 \mathrm{~Hz}$, respectively. 69 
Figure 3.26 Plot of non-dimensional dynamic roughness strength versus Reynolds number at a pitching rate with a reduced frequency of 0.1 ........................................................ 71

Figure 3.27 Comparison between (a) two-dimensional and (b) three-dimensional simulation results of an airfoil undergoing rapid pitching at $\mathrm{Re}=150,000$ and $\mathrm{k}=0.1$........ 73

Figure 3.28 Streamlines shaded by vorticity magnitude, showing span-wise flow within the LEV region. 74

Figure 3.29 Three dimensional streamline comparison of a clean airfoil with an airfoil with actuated dynamic roughness.................................................................................. 75

Figure 3.30 Iso-surface of a single vorticity magnitude contoured by chord-wise velocity component for a (a) clean airfoil at $11.76^{\circ}$ angle of attack and a (b) airfoil with actuated dynamic roughness at the same angle of attack. ...................................... 76

Figure 4.1 Comparison of PIV results with those collected via CFD. ...................................... 78

Figure 4.2 Comparison of a (a) phase averaged and an (b) instantaneous PIV calculation..... 79 


\section{LIST OF TABLES}

Table 2.1 Conditions used to observe changes in LEV development with respect to Re........ 30

Table 2.2 Typical sources of error for PIV measurement............................................... 37

Table 2.3 Principal dimensions of PIV measurement...................................................... 38

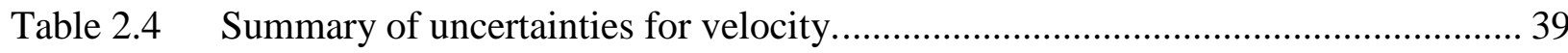

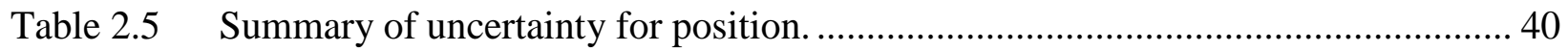

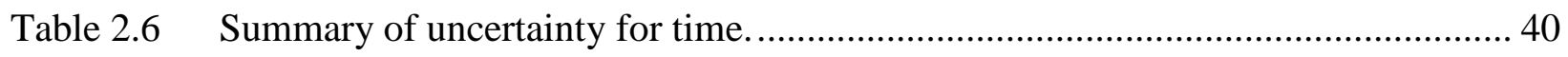

Table 3.1 Pitching parameters used for mesh comparison, pitching method comparison, and

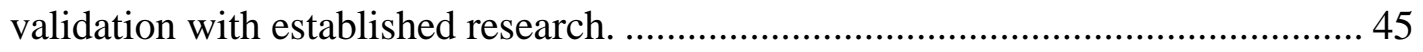

Table 3.2 Properties of different grids used for refinement study. ................................... 51

Table 3.3 Tabulated data comparing dynamic roughness strength with Reynolds number at a

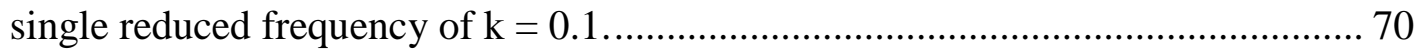




\section{CHAPTER 1: INTRODUCTION}

\subsection{Overview}

Dynamic stall has been a subject of much research for decades, and still its details are yet to be fully understood. The reason for the large amount of interest in dynamic stall is due to the fact that it occurs in many different types of applications. It is a major factor when dealing with helicopter rotor blades, wind turbines, and high maneuvering military aircraft. The recent trend mimicking biological flight (biomimetics) has correlated dynamic stall to the aerodynamics observed in flapping flight. Dynamic stall is associated with an airfoil, wing, or even the blade of a turbo machine that encounters a rapid pitching motion. It is, in fact, a dynamic delay of stall to angles significantly beyond the static stall angle. A key characteristic of dynamic stall is the formation of a vortex near the leading edge, often referred to as the leading edge vortex (LEV) or simply as the dynamic stall vortex (DSV). The formation of this vortex is the source of extra lift associated with dynamic stall. This extra lift is lost as the vortex sheds into the wake and causes an increase in drag as well as a large shift in pitching moment [1]. The first evidence of dynamic stall was identified on helicopters. Helicopter design engineers were unable to predict the performance of high speed helicopters using conventional aerodynamics. This led to increased emphasis on unsteady aerodynamics and their effects [2]. McCroskey [3] noted in his text the importance of understanding the unsteadiness encountered in pitching airfoils, especially oscillating airfoils. He suggests that a large hysteresis develops in the fluid-dynamic forces and moments as the airfoil oscillates and the behavior of lift, drag, and that pitching moment cannot be grasped without fully investigating the unsteady motion of the airfoil. Figure 1.1 [2] presents an evolution of the processes that occur during the cycle of a pitching airfoil. This evolution includes the airfoil pitching down after the initial rapid pitch up. 


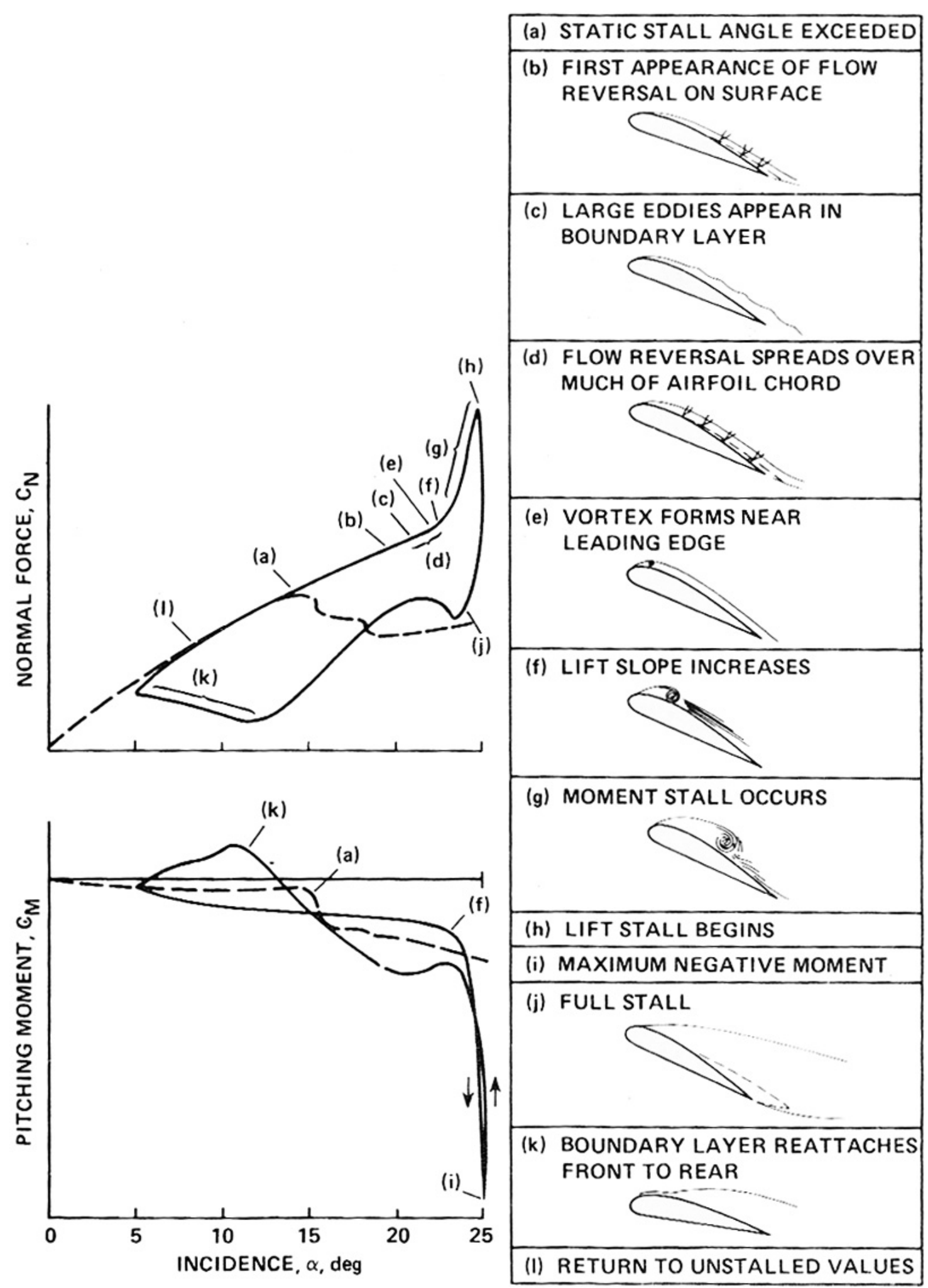

Figure 1.1 Events of dynamic stall on NACA 0012 airfoil [2]. 
Since the discovery of dynamic stall, research has been conducted to not only gain an understanding of the physics occurring, but also to find ways to alter the formation of the LEV. With the ability to alter the LEV one could further expand the operational envelope of the aircraft. Most of the strategies used to control the LEV originate from research involving laminar boundary layer control. Laminar boundary layer control attempts to inhibit the transition from a laminar boundary layer to a turbulent boundary layer, as well as prevent or enhance flow separation. Skin friction drag can be reduced up to an order of magnitude if the boundary layer is in a laminar state, which correlates to longer range, less fuel consumption, and perhaps increased speed [4]. Some typical boundary layer flow control techniques include classics such as suction, injection, vortex generators, roughness, compliant surfaces; and also recent techniques such as plasma actuators and synthetic jets.

Although these techniques show great promise in research settings there are some common reasons why they are not utilized in practice. A common problem in flow control is that the device actually causes a net increase in energy expenditure. This usually stems from the fact that the device uses more energy than is saved by the increase in aerodynamic efficiency. Also, many of these techniques are only worthwhile at a certain performance condition; while at other times may cause adverse effects. Therefore, a proper flow control apparatus used to alter the LEV occurring during dynamic stall should be tunable to operate at a variety of conditions, such as various Reynolds number $(R e)$ and pitching frequencies.

\subsection{Dynamic Roughness at Static Angle of Attack}

Recent research at West Virginia University (WVU) has shown that dynamic roughness (DR) can eliminate the leading edge separation bubble of an airfoil at a constant angle of attack [5] [6]. This has been proven, at least at the relative low Reynolds numbers studied, both computationally and experimentally. This research was preceded by work done by Huebsch, et al. [7] [8] [9]. The work done by Huebsch, et al. initially dealt with the effects of small-scale roughness on leading edge laminar separation. This then evolved into evaluating the effects of small scale dynamic roughness. Since their work was focused on leading edge separation, the leading edge was modeled by a conformal mapping of a parabolic leading edge. This reduced the computational requirements while allowing a highly resolved and well behaved grid in the 
separation region. Their work showed elimination of leading edge separation by actuation of small scale (sub boundary layer height) dynamic roughness. The work done by Gall [5] and Huebsch et al. [6] expanded this work by modeling dynamic roughness on a NACA 0012 airfoil using the commercial CFD package Fluent. This research is the predecessor to the research detailed in this document, and also the motivation behind evaluating the ability for dynamic roughness to alter the LEV of a pitching airfoil. The problem of leading edge separation occurs on airfoils or wing sections producing lift at angle of attack and Reynolds numbers low enough for the adverse pressure gradient to cause separation. Mueller and Batill provide a description of the separation bubble, "The leading edge separation bubble is formed when the laminar boundary layer separates from the surface as a result of the strong adverse pressure gradient downstream of the point of minimum pressure. This separated shear layer is very unstable and transition usually begins a short distance downstream of separation. After transition from laminar to turbulent flow, the turbulent shear stresses energize the shear layer by entraining fluid from the external stream. Reattachment occurs when the pressure is nearly equal to a value which would exist if there had been a turbulent boundary layer over the airfoil with no separation bubble present. The region between separation and reattachment is referred to as the separation bubble” [10].

Figure 1.2 shows an example of the success found in the three-dimensional numerical analysis using dynamic roughness to eliminate the leading edge separation bubble by [5]. One can see the reduction in circulation and thus increase in suction pressure due to the presence of dynamic roughness. 

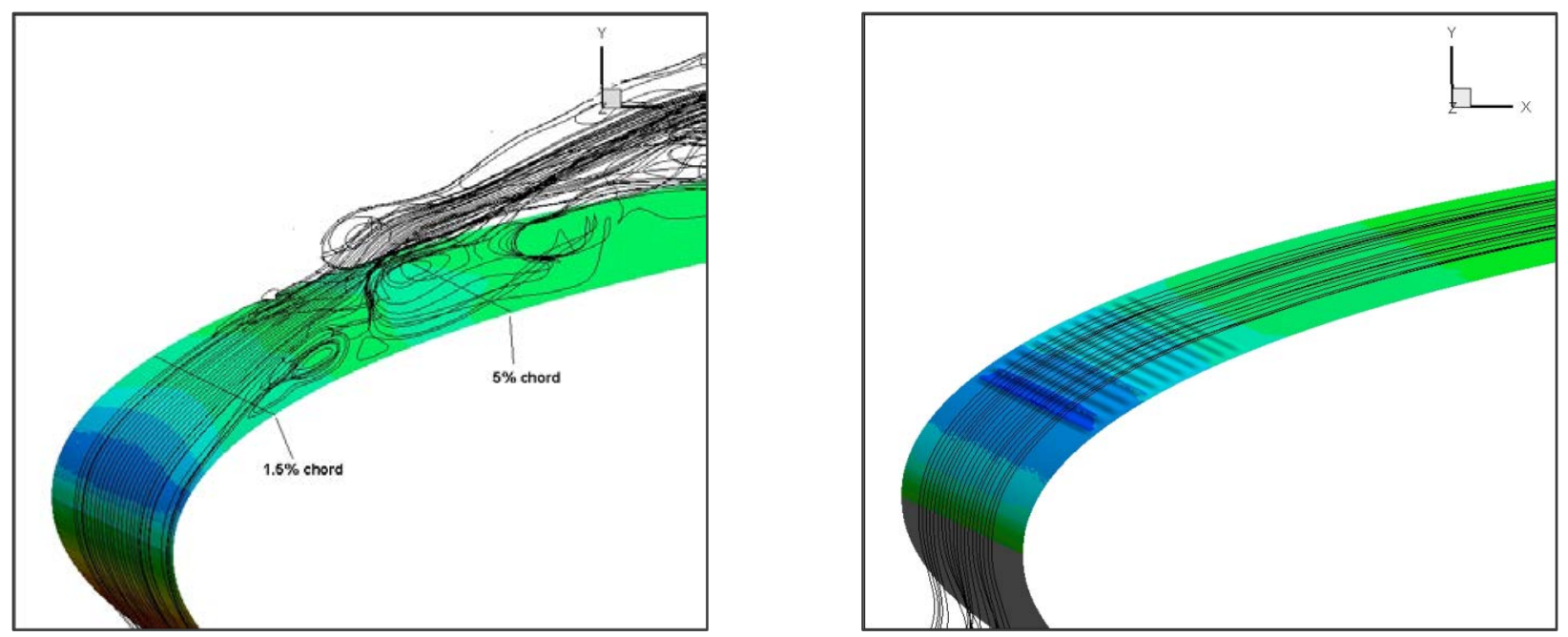

Figure 1.2 Surface pressure contour and pathline plots of a clean airfoil and an airfoil with dynamic roughness ridges, respectively, at $12^{\circ}$ angle of attack and 100,000 Reynolds number [5].

Success was also found experimentally using both flow visualization as well as surface pressure data, as shown in Figure 1.3. The previous success with eliminating the leading edge separation bubble using dynamic roughness is the basis for evaluating whether it can also alter the LEV of a rapidly pitching airfoil.
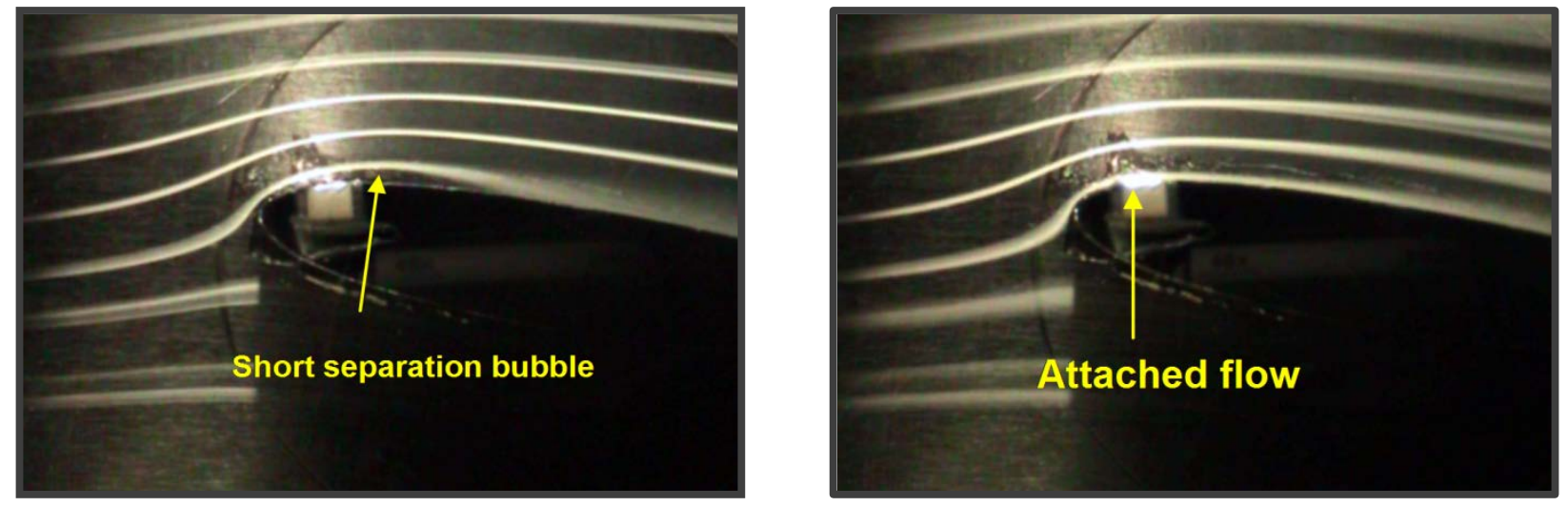

Figure 1.3 Smoke visualization of a NACA 0012 airfoil at $9.5^{\circ}$ angle of attack and 100,000 Reynolds number with no dynamic roughness and with dynamic roughness active [5].

\subsection{Previous Numerical Analysis of Dynamic Stall}

Mehta and Lavan [11] studied the laminar unsteady flow around an impulsively started airfoil at angle of attack and low Reynolds number $\left(R e_{c}=1000\right)$ by solving the Navier-Stokes equations in the whole flow field. They noticed the change in position of the front and rear stagnation points, as well as the correlation between the change in lift and the growth of the separation bubbles and 
vorticity. They also associated the "bursting" of the large leading edge bubble with airfoil stall. Shida, et al. [12] accounted for compressibility in their simulations of a pitching NACA 0012 airfoil. They matched their results to experiments by McCroskey and Pucci [13], which evaluated oscillating airfoils at much higher Reynolds number $\left(R e_{c}=4 \times 10^{6}\right)$ than previously analyzed numerically. In particular, [12] compared lift coefficient and moment coefficient hysteresis acquired numerically to that found in experiment. They found it important for the mesh density to be fine enough to capture the small vortices along the airfoil surface which produce a pressure coefficient curve indicating attached flow.

Visbal and Shang [14] investigated the flow around a rapidly pitching airfoil at a Reynolds number of $10^{4}$. The provided a sequence of flow events that occur during the pitch-up maneuver, as shown in Figure 1.4. The development of the leading edge vortex is due to the separation point on the upper surface of the airfoil moving upstream as the airfoil is pitched up. Eventually, the separation region reaches the leading edge area, where the separated shear layer reattaches. This divides the upper surface flow into two vertical structures, the LEV and the shear layer vortex. The LEV continues to grow in size and its center is displaced downstream until it detaches, approximately at the midchord location. They also investigated the effects of pitching rate and pitching axis on the flow field structure. They were able to show LEV development is delayed as pitch rate is increased, LEV development can also be delayed by displacing the pitching axis downstream. 

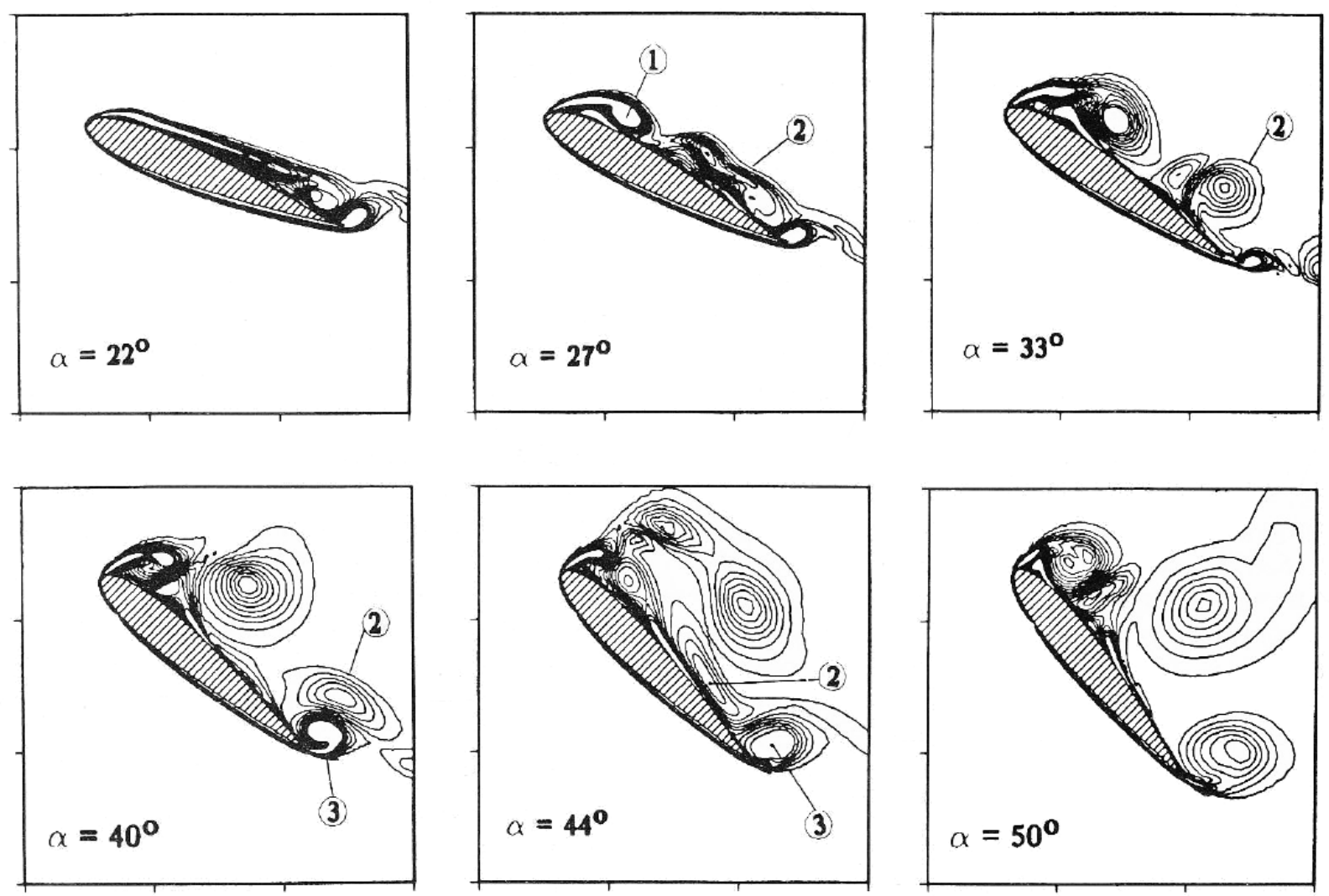

Figure 1.4 Evolution of vorticity field: 1) leading-edge vortex, 2) shear layer vortex, 3) trailing-edge vortex [14].

Visbal [15] did additional research concerning the effects of compressibility on dynamic stall. He concluded the main effects to be a change from trailing-edge stall to leading edge-stall and a reduction in the stall delay and maximum lift. Shih, et al. [16] performed a computational study on a pitching airfoil using the discrete vortex, random walk approximation in tandem with experimental Particle Image Velocimetry (PIV) work done in a water towing tank. The flow regime they focused on was a chord Reynolds number of 5000. The main objective of their study was to evaluate the ability of PIV to study unsteady flows as well as their computational method. They produced numerical results that correlated well with their experimental work, giving confirmation to both methods. Choudhuri, et al. [17] compared results for structured and unstructured meshes for a NACA 0012 airfoil undergoing dynamic stall as well as two separate numerical algorithms. They investigated the unsteady leading-edge boundary layer separation and also gave a classification of critical points as seen in plots of the instantaneous streamlines in Figure 1.5. They found close agreement between the structured and unstructured computations 
confirming the accuracy of their results. The results from [17] were used to validate the results from the current computational research.
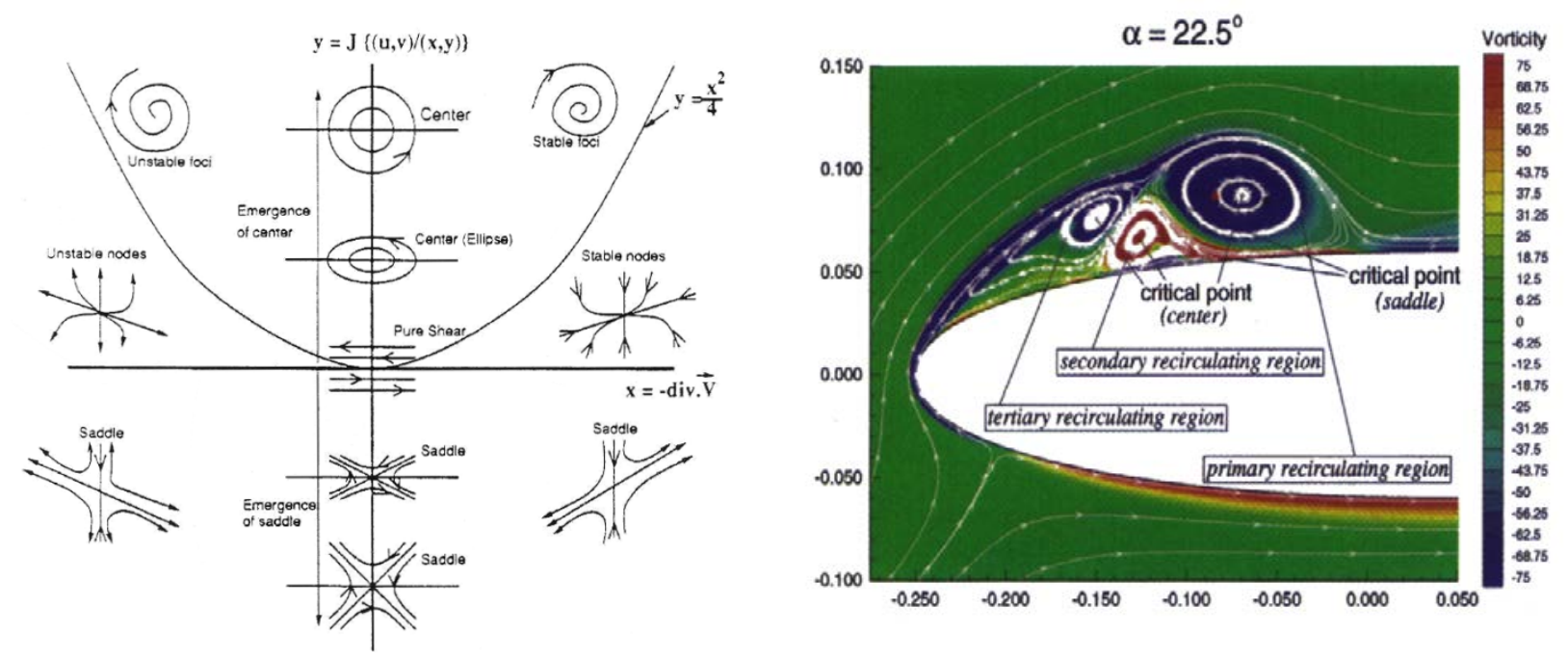

Figure 1.5 Classification of critical points and corresponding location in the computed flow field [17].

Rothmayer and Bhaskaran [18] noted the development of Rayleigh instabilities in dynamic stall prior to first flow reversal. Their study was restricted to an idealized leading edge, or flow past a parabola. They documented that these Rayleigh instabilities produce a complex cascading of two-dimensional secondary instabilities. Suito, et al. [19] used a multi-directional finitedifference method to analyze the separating strong shear layer during dynamic stall of a NACA 0012 airfoil at a chord Reynolds number of $5.0 \times 10^{5}$. They concluded, "The vortex structures which give rise to the dynamic stall are the same in spite of the differences such as Reynolds number or airfoil motion”. Huebsch and Rothmayer [20] investigated the effect of surface roughness on dynamic stall, and particularly simulated ice roughness. They concluded roughness whose characteristic height was smaller than that of the boundary layer height affected the secondary separation mechanism, and also that large-scale roughness can "significantly alter the inception time for the formation of the dynamic-stall vortex (leading edge vortex)”.

Further research was performed by Akbari and Price [21] to investigate the effects of pitching frequency and Reynolds number on an oscillating airfoil. They showed that pitching frequency had the most effect on the flow field and the force moments. With higher frequencies flow separation was delayed, which caused the peak lift to occur at a higher angle of attack. The 
increase in pitching frequency also caused an increase in the "negative damping in the pitching moment coefficient hysteresis loop". It is significant to be able to associate changes in such characteristics with seemingly secondary effects. This confirmed earlier results found by [14].

All computational research mentioned up to this point has been two-dimensional in nature. Spentzos, et al. [22] performed three-dimensional computational analysis of dynamic stall. They concluded that similarity between two-dimensional and three-dimensional CFD analysis is good only in the mid-span area of a wing. The outboard section is sufficiently affected by the interaction between the LEV and the tip vortex. Visbal [23] performed three-dimensional analysis using an implicit large-eddy simulation (ILES) approach. He focused this study to low Reynolds number conditions $\left(R e_{c} \leq 6 \times 10^{4}\right.$ ) of a plunging airfoil with relatively high frequency and low amplitude. It was shown that although at inception the LEV is laminar, it quickly experiences an abrupt breakdown and undergoes transition. In a follow up study Visbal [24] broadened the flow characteristics by lowering the frequency and increasing the plunging amplitude, relevant to more micro aerial vehicle (MAV) applications. He was able to show for certain Reynolds number regimes the flow will remain effectively laminar throughout the plunging cycle $\left(\operatorname{Re}_{\mathrm{c}} \approx 1 \times 10^{3}\right)$. At around $\operatorname{Re}_{\mathrm{c}}=5 \times 10^{3}$ transitional effects are present, while at $R e_{c}=1.2 \times 10^{5}$ a single turbulent LEV is formed.

\subsection{Previous Experimental Analysis of Dynamic Stall}

Martin, et al. [25] performed an experimental study of dynamic stall on a two-dimensional NACA 0012 airfoil. Their data consisted of surface pressure and hot-wire measurements, as well as smoke flow visualization. They focused on the effects of Reynolds number, oscillation amplitude, and oscillation frequency. It was concluded that for the range $\operatorname{Re}_{c}=10^{6}$ to $3 \times 10^{6}$, the angle of attack required for stall initiation decreases with increasing Reynolds number while the angle of attack needed for maximum lift increases with increasing Reynolds number. The authors noted that previous research performed at much lower Reynolds number did not show the same relationship between Reynolds number and stall initiation. This is significant to find that perhaps dynamic stall behaves differently at different Reynolds numbers. 
A supplement to the previous research was done by McCroskey, et al. [26] Their work focused more on boundary layer separation, general effects of vortex shedding at different oscillation frequencies, and the effect of leading-edge geometry on the normal force and pitching moment coefficients. The leading edge alterations they studied consisted of the ONERA Extension Cambre airfoil (used to study the effects of camber), symmetrical extensions to reduce the leading edge radius, leading edge serrations, and boundary layer trips. This research showed contrary to previous assumptions, "the shed vortex appears to be fed its initial vorticity by the abrupt, unsteady separation of the turbulent boundary layer, not the laminar bubble”. This conclusion is particularly significant to the research proposed here since the dynamic roughness is known to alter the leading edge laminar bubble. Walker, et al. [27] studied the effects of pitching rate on flow separation encountered during dynamic stall. Their data consisted of smoke visualization as well as near wall hot-wire velocity measurements. They were able to confirm that an increase in pitching rate translated into a delay in flow separation and "more energetic" suction peak and LEV. Figure 1.6 shows the delay in flow separation and LEV development as the non-dimensional pitching rate is increased from 0.2 to 0.6 using smoke flow visualization. These results are consistent with previous computational research. 


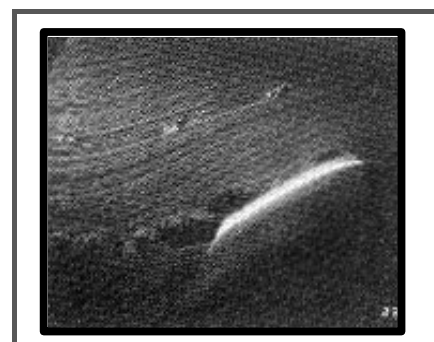

$\alpha=24^{\circ}$

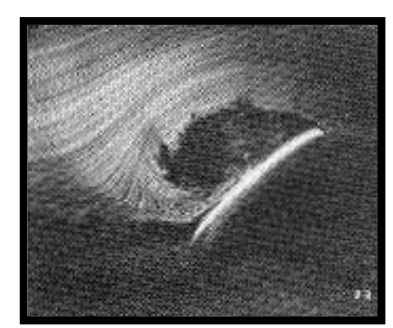

$\alpha=36^{\circ}$

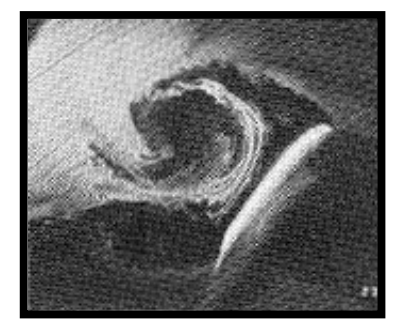

$\alpha=47^{\circ}$

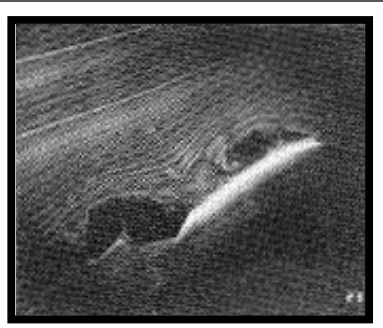

$\alpha=27^{\circ}$

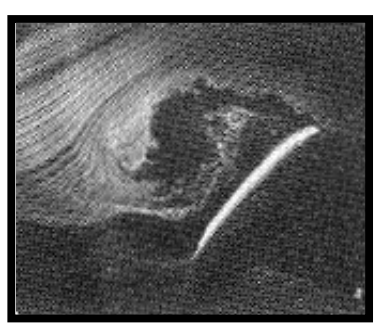

$\alpha=40^{\circ}$

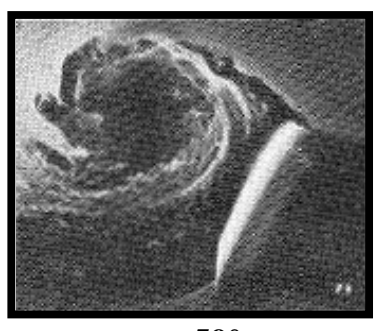

$\alpha=59^{\circ}$

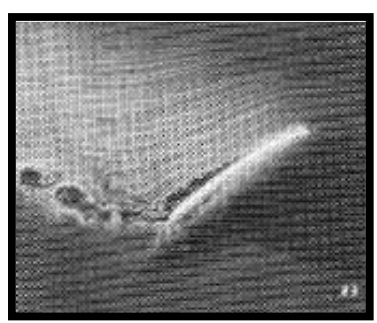

$\alpha=28^{\circ}$

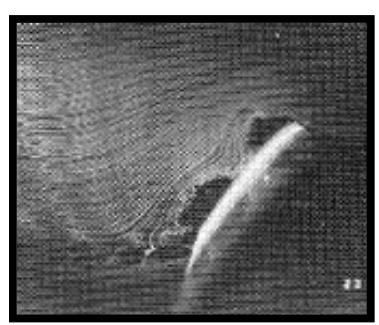

$\alpha=47^{\circ}$

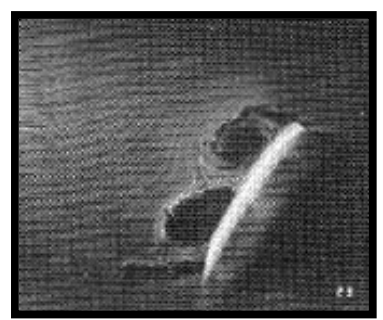

$\alpha=56^{\circ}$

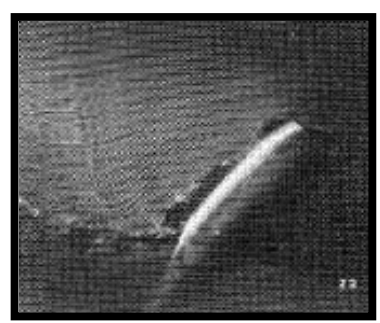

$\alpha=42^{\circ}$

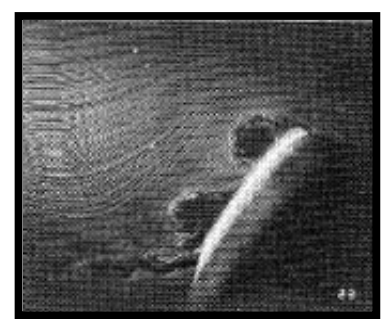

$\alpha=52^{\circ}$

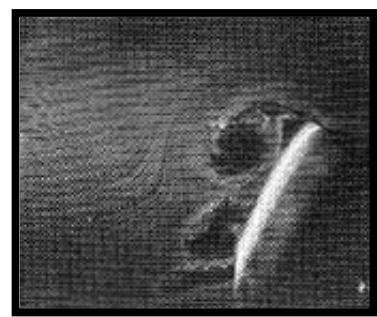

$\alpha=60^{\circ}$

(a)

(b)

Figure 1.6 Flow visualization for $R e_{\mathrm{c}}=45,000$ and constant pitching rate with a non-dimensional pitching rate of $0.2(a)$ and $0.6(b)$ [27].

Brandon [28] studied the effects of dynamic stall on the aerodynamic characteristics of high performance aircraft. He did wind tunnel testing of a representative fighter aircraft and investigated dynamic effects on lateral stability and available control power during control surface deflections. Brandon concluded that the results seen in simple two dimensional airfoils are similar to those found on a realistic three dimensional aircraft configuration. He also noted that the dynamic lift effects did not significantly alter turn performance, but did have a significant impact on longitudinal and lateral stability. Chandrasekhara and Ahmed [29] recorded laser velocimetry data for an oscillating airfoil. They captured rapid accelerations over a large region of the airfoil with values as high as $1.6 U_{\infty}$ (free stream velocity) as well as wall effects as far away as half the chord length from the airfoil. These rapid accelerations seem to be the source of the increased suction pressure and, therefore, increased lift. To better understand the evolution of the LEV, Acharya and Metwally [30] performed detailed surface pressure 
measurements of pitching airfoils. They identified three regions based on the surface pressure plots: a suction peak in the region near the leading edge (LESP), a constant pressure region or pressure plateau (CPP), and a suction peak associated with the dynamic stall vortex (DSVP). Acharya and Metwally discovered significant differences in the evolution of these regions dependent on whether the pitch rate was relatively "low" are "high". This gave further evidence on the importance of pitch rate in the development of dynamic stall.

Ahmed and Chandrasekhara [31] focused on the down stroke of an oscillating airfoil which encounters dynamic stall on the upstroke. They used the Schlieren method for qualitative analysis of the global flow field, laser Doppler velocimetry (LDV) for velocity measurements, and point diffraction interferometry (PDI) for measuring density and pressure distributions. They were able to identify the flow reattachment process, which begins when the airfoil is very close to the static stall angle on its downward stroke and corresponds to a large rise in the suction peak. Shih, et al. [32] performed further research, but this time focusing on the flow near the leading and trailing edges. They used PIV to detail the formation of the vortices and their interaction with the boundary layer vorticity. They concluded that the trailing edge flow field only plays a "secondary role” on the dynamic stall process.

Although Bousman [33] himself did not perform an experimental analysis, he did analyze previous dynamic stall data to characterize the relationship between augmented lift, negative pitching moment, and increase in drag, particularly for rotorcraft. He concluded this relationship shows little sensitivity to airfoil profile and Mach number; and is independent of Reynolds number, oscillation frequency, and blade sweep. He highlights the need for the development of multi element airfoils or variable geometry airfoils that can take advantage of the increase in performance occurring during dynamic stall, but prevent the disadvantages such as increased drag and changes in pitching moment. Schreck, et al. [34] conducted shear stress measurements on a NACA 0015 airfoil as it was pitched at a constant rate through dynamic stall. Using the shear stress calculations they identified boundary layer flow reversal preceding eventual LEV formation and convection downstream. Geissler and Haselmeyer [1] studied the effect of transition on dynamic stall onset. They used a variety of tripping devices to study a forced transition flow encountering dynamic stall and concluded the development, shedding, and 
accumulating of vorticity plays the dominant role in both free transition as well as forced transition.

\subsection{Dynamic Stall and Biological Flight}

Man's first attempts at flight involved replicating nature's flyers, which included flapping wings to not only produce lift, but also propulsion. Things have come full circle with a relatively recent renewed interest in flapping flight. Researchers are again studying nature's flyers to find more efficient means for flight, particularly for smaller aircraft such as unmanned aerial vehicles (UAVs) and micro aerial vehicles (MAVs). The following is a brief review of some research that relates dynamic stall with biological flight.

Ellington, et al. [35] performed smoke flow visualization of a hawkmoth to better understand the mechanism of lift production in flapping flight insects. According to the laws of conventional dynamics, insects should not be able to fly. The source of the extra lift for this type of flyer remains elusive. They were able to observe an "intense” LEV on the down-stroke, suspected to be the source of the extra lift. They concluded this vortex is created by dynamic stall and is held stable by the convection of the vorticity to the wingtip. This observation was confirmed by work done by Liu, et al [36]. They performed three-dimensional computational fluid dynamics on a hovering hawkmoth flapping wing. Their results showed the LEV evolution and again the axial flow component of the vorticity. The negative pressure region created by the LEV is responsible for lift enhancement. Figure 1.7 presents the computational results from [36] along with the smoke visualization captured by [35]. One can clearly see the formation of the LEV and its convection down the span to the wingtip. 


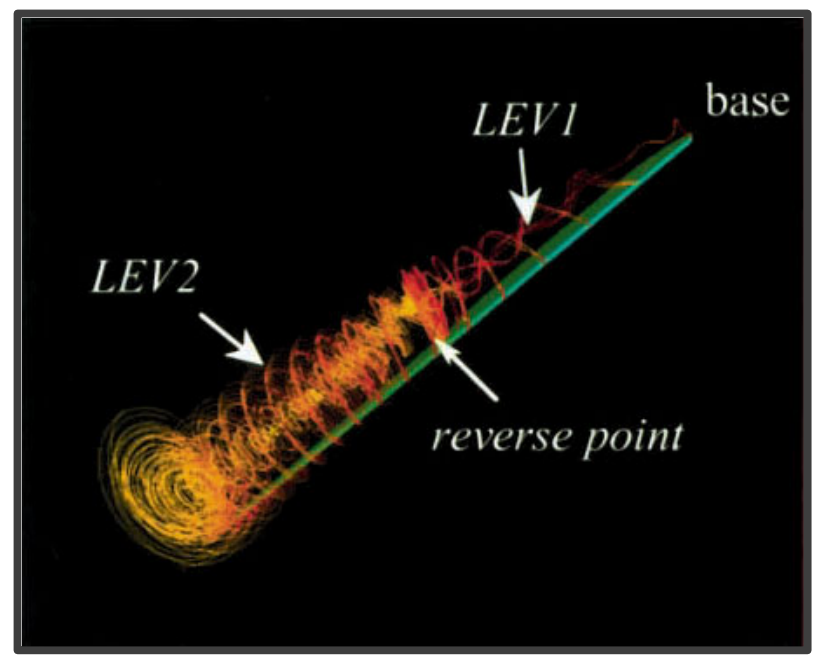

(a)

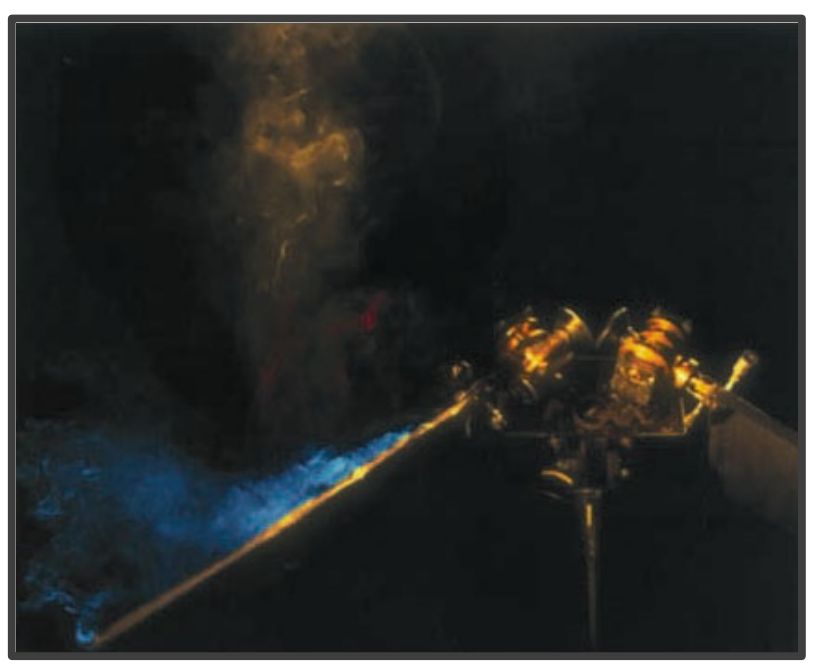

(b)

Figure 1.7 Comparison of computational work done by Liu, et al. (a) [36], with smoke visualization done by Ellington, et al. (b) [35].

Work by Birch and Dickinson [37] further investigated the mechanism that keeps the LEV attached in insect flight. The prevailing hypothesis at the time was a spiral vortex which gained energy from a vortex core, analogous to delta wing aerodynamics. Birch and Dickinson concluded at Reynolds numbers encountered at typical insect flight, "flapping wings do not generate a spiral vortex akin to that produced by delta wing aircraft”. They did further investigation on whether limiting the spanwise flow with fences or baffles would cause detachment of the LEV, but this was not the case. From this research they proposed a different hypothesis that the downward flow induced by tip vortices is what limits the growth of the LEV.

Srygley and Thomas [38] trained red admiral butterflies to fly to and from artificial flowers in a wind tunnel. They performed smoke flow visualizations during butterfly takeoff, forward flight, and landing. The LEV was shown to create large flow accelerations to increase lift for vertical takeoff as well as to accelerate while in forward flight. Lu, et al. [39] performed particle image velocimetry (PIV) on a flapping model dragonfly wing in hovering conditions. They were able to record the existence of a dual vortex formed on the down-stroke using PIV. They hypothesized that secondary separation may play an active role in the minor vortex formation, while the separated boundary layer at the leading edge was rolling up to form the primary vortex. The dual LEV also seemed to be independent of wing aspect ratio. Lu and Shen [40] followed this up with additional research identifying one major vortex and three minor vortices making up the LEV system on a three dimensional flapping wing. 
Muijres, et al. [41] studied slow flying bats utilizing PIV. They observed lift enhancement as much as $40 \%$ by way of the LEV. This research was important in showing that lift enhancement by means of leading edge vorticity is not limited to insects, but also larger flyers. Lua, et al. [42] performed an interesting study concerning the use of flexible vs. rigid wings for flapping flight. The primary purpose of their study was to evaluate the lift force generation created when using a rigid wing as opposed to a flexible wing. They found that there is a critical stiffness coefficient, and if the wing properties fall below this critical point (wing is too flexible) lift generation is reduced. The interesting and relevant result to this research was that they attributed the loss in lift generation to the altering of the LEV due to the motion of the "too flexible" wing. Jones and Babinsky [43] recorded PIV, flow visualization, and force data for a "waving” wing. The waving wing is a three-dimensional simplification of normal flapping flight observed in nature. The purpose of their study was to evaluate the flow characteristics over a range of Reynolds number $\left(R e_{c}=10,000\right.$ to 60,000$)$. The flow structures over the wing did not appear to change with regards to Reynolds number, but the leading edge vortices appear to grow and shed more quickly at lower Reynolds number, based on a non-dimensional time-scale.

\subsection{Previous Research Concerning the Alteration of the Leading Edge Vortex}

As stated above, flow control techniques for dynamic stall are based on their static stall counterparts, i.e., suction, blowing, moving walls. Freymuth et al. [44] recorded smoke flow visualizations of a pitching airfoil with a rotating leading edge. They were able to show elimination of the LEV and documented a minimum circumferential speed needed by the leading edge cylinder to maintain control. This minimum speed was dependent on free stream velocity and pitching rate. Increasing the speed beyond this minimum had no negative effect on separation control. Figure 1.8 is a sample of the results provided by [44]. One can clearly see the elimination of the LEV and dynamic stall by leading edge rotation. 


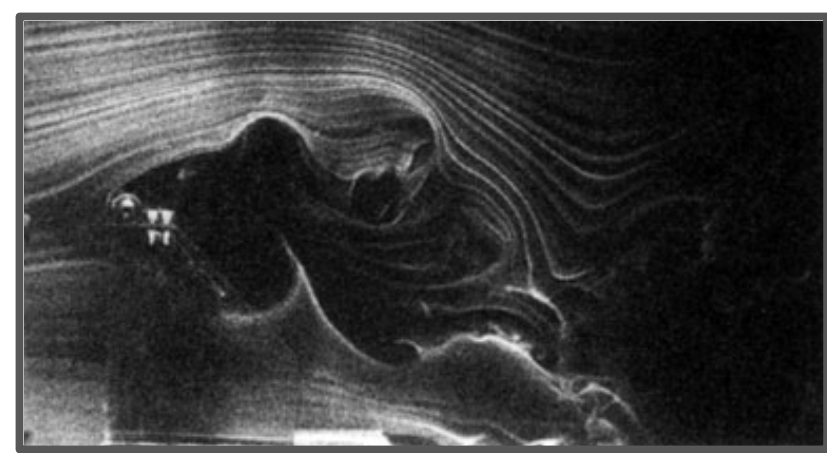

(a)

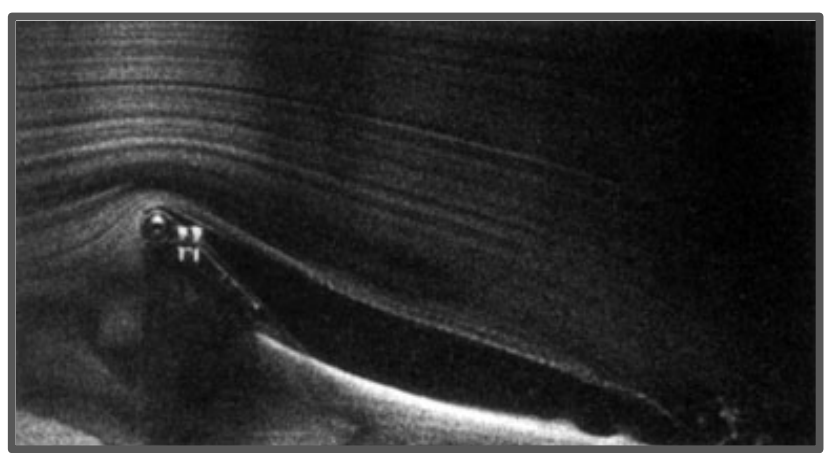

(b)

Figure 1.8 Smoke flow visualization from Freymuth, et al. [44] showing the effect of flow control on a pitching airfoil using a rotating cylinder leading edge. Both airfoils are at $40^{\circ}$ during their pitching evolution, without nose rotation (a) and with nose rotation (b).

Visbal [45] performed computational analysis to study the effects of leading edge suction via boundary layer bleed and tangential surface motion to delay separation and thus delay the onset of the LEV. They were able to show control using both methods. Distributed suction seemed to be more effective than a concentrated suction slot on the airfoil upper surface. A summary of the results from [45] are shown in Figure 1.9.

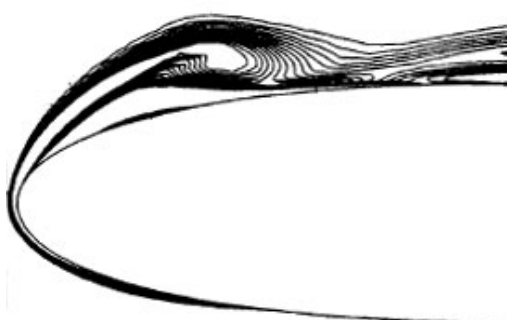

(a)

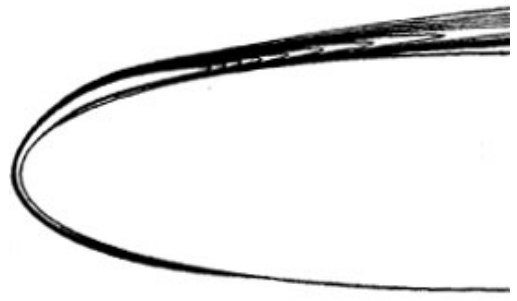

(b)

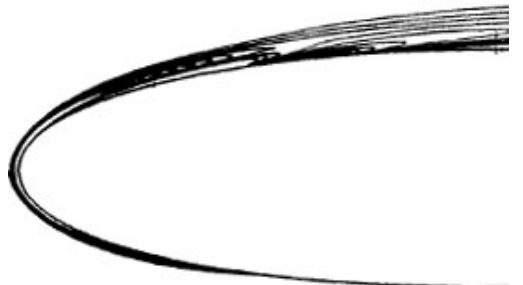

(c)

Figure 1.9 Computational results by Visbal [45] of a pitching airfoil at $36^{\circ}$ in its pitching evolution with (a) no control techniques, (b) distributed surface suction, and (c) a moving wall surface.

Karim and Acharya [46] performed experimental analysis of LEV control by means of leading edge surface suction. They were able to derive a required suction rate needed to provide adequate control of the LEV. This was done by balancing the rate of suction with the reverseflow accumulation rate. Figure 1.10 is a plot from [46] that provides the suction requirements for different dimensionless pitch rates. With this plot, one can determine the suction requirements during a constant pitch rate maneuver by following a line of constant $\alpha^{+}$. It is also evident from this plot that to retain complete suppression of the LEV the suction flow rate needs to be increased as the angle of attack increases. 


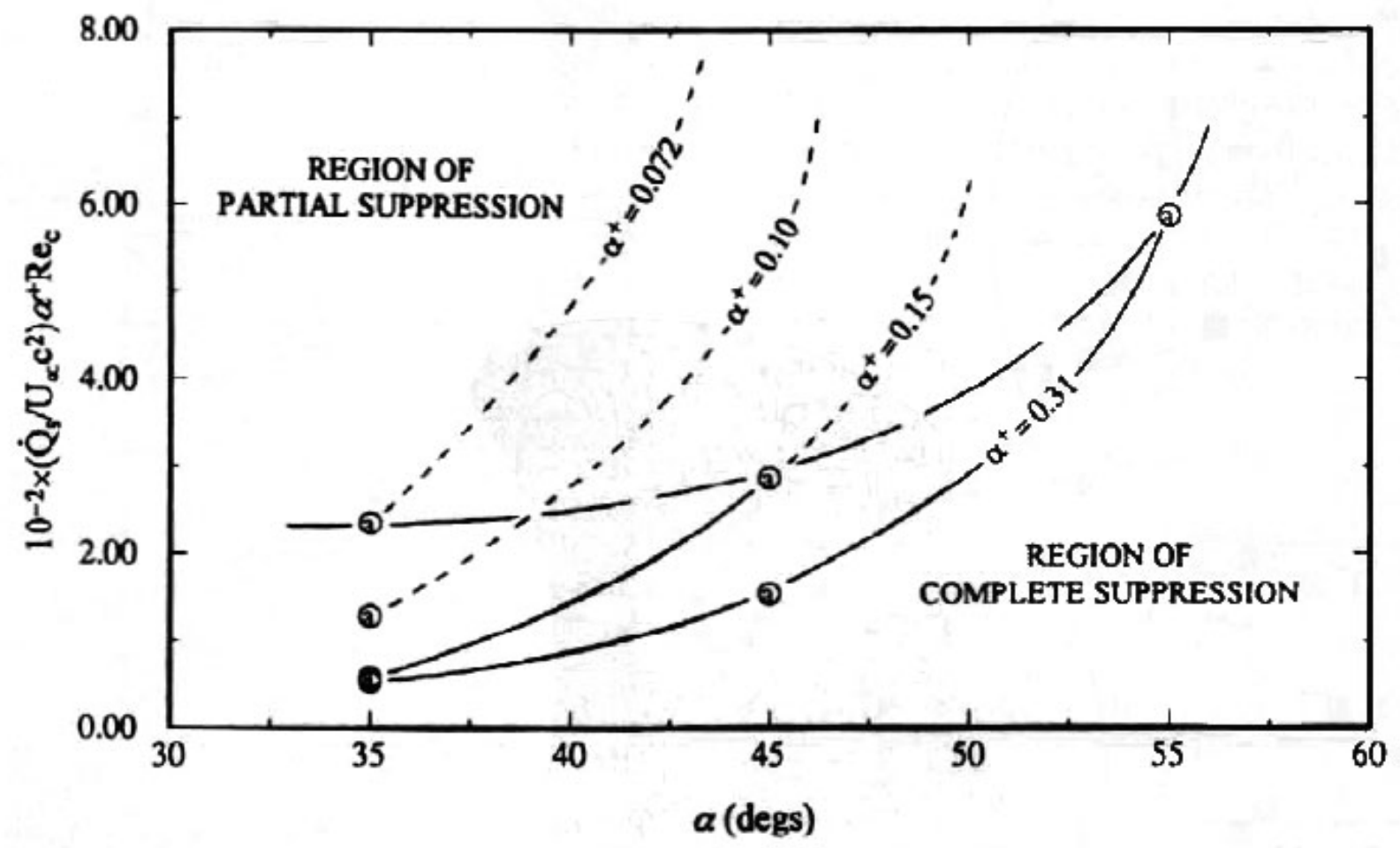

Figure 1.10 Variation of suction flow rate for complete suppression of the LEV with angle of attack, for different pitch rates [46].

Post and Corke [47] evaluated the use of a plasma actuator to alter the development of the LEV on an oscillating NACA 0015 airfoil. They used smoke flow visualization as well as surface pressure measurements to document the plasma actuator effectiveness. Three plasma actuation scenarios were studied: open-loop quasi-steady, open-loop unsteady, and closed-loop steady. The closed-loop actuation scenario would energize the plasma actuator based on airfoil angle of attack. They did this to investigate the ability to allow the LEV to form, but perhaps prevent dynamic stall and also to aid reattachment during the down stroke of the airfoil. It was found that all three scenarios improved the lift cycle, with steady actuation increasing lift over most of the cycle, except at the peak angle because it suppressed the LEV, although a side effect of suppressing the LEV is the elimination of the sharp drop in lift coefficient at the start of pitch down. The unsteady plasma actuation improved the lift most notably on the down stroke and the closed-loop control algorithm showed greatest improvement with regards to integrated lift. Greenblatt [48] evaluated periodic forcing as a means to control dynamic stall. He used surface pressure and wake measurements to evaluate their effectiveness and oriented the blowing slots at $45^{\circ}$ and $90^{\circ}$ to the chord-line. More success controlling dynamic stall was achieved using the 
blowing slot oriented $45^{\circ}$ to the chord-line and is attributed to "trapping" the bubble upstream of the forcing slot location, but relatively large forcing amplitudes were required to gain control.

The previous research done by Huebsch [9] on dynamic roughness is the main motivation for the current study. Using a two-dimensional Navier-Stokes numerical simulation on a parabola, also used by [18] [20], he was able to delay the formation of the LEV using dynamic roughness actuation. This, in turn, can translate into the airfoil reaching a higher angle of attack before dynamic stall occurs. The delay in the formation of the LEV is shown in Figure 1.11.
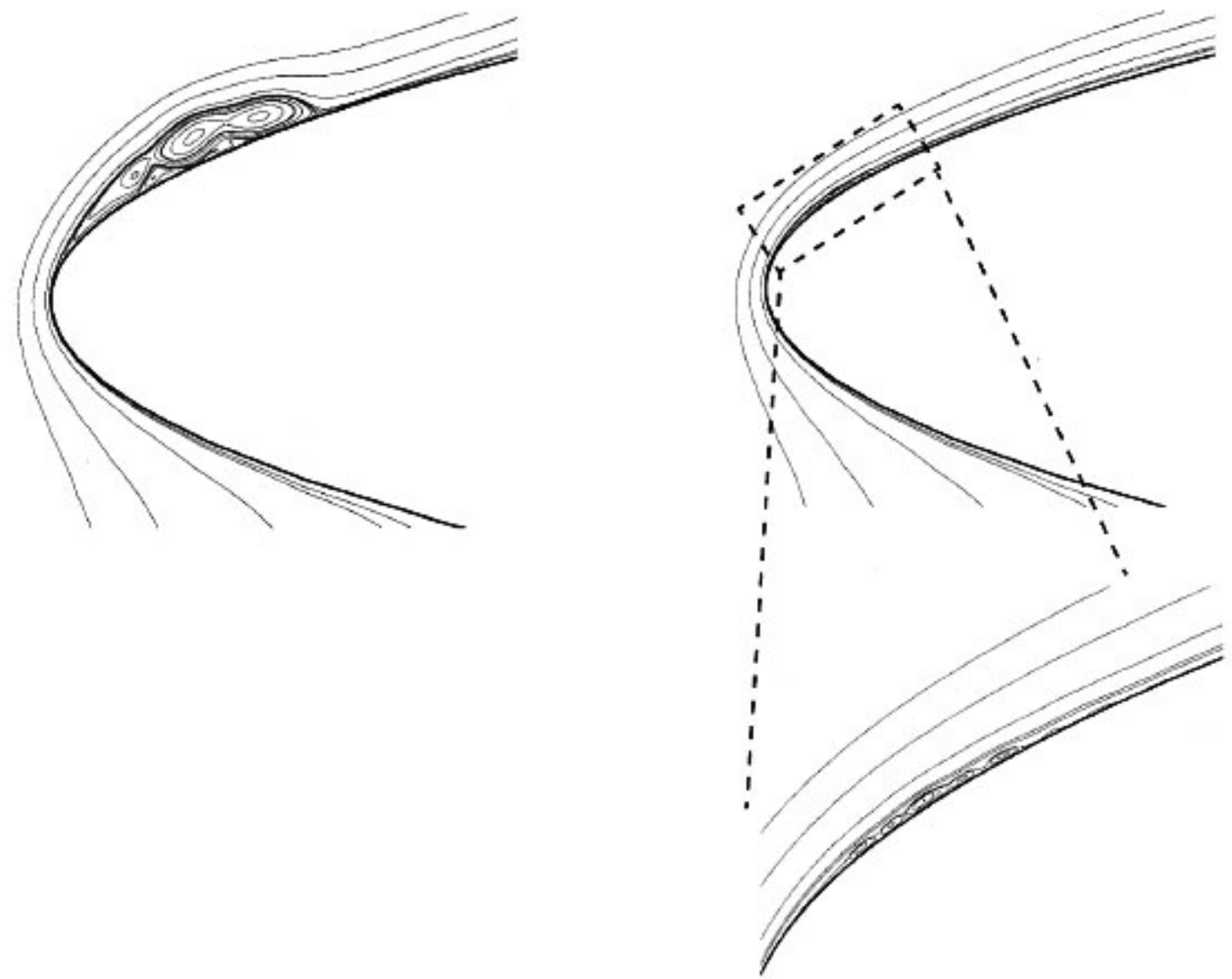

(a)

(b)

Figure 1.11 Results from work done by Heubsch [9] showing the LEV on the leading edge of a clean parabola (a) and the effect of dynamic roughness (b), all at the same physical time step and angle of attack. 


\subsection{Objectives}

As shown through previous research LEV alteration, predominantly LEV development delay, has been established through typical laminar boundary layer control techniques. Dynamic roughness has been shown both computationally and experimentally to be a viable laminar boundary layer control technique, at least in steady aerodynamics [5] [6] [9]. It was the goal of this research to expand the applicability of dynamic roughness to alteration of the LEV development on a rapidly pitching airfoil, an unsteady phenomenon. Both a numerical and experimental analysis was conducted to help validate the results and represents the first time such an approach has been used to evaluate the application of dynamic roughness in such an unsteady aerodynamic phenomenon. In the pursuit of this main objective a few other aspects of LEV development were investigated to help validate the results, as well as provide further evidence for previously debated results. A bulleted list is given to outline the objectives sought in the research.

- Can dynamic roughness alter the development of a LEV on a rapidly pitching airfoil? Particularly, do conditions exist that show dynamic roughness can both delay LEV development to higher angles of attack as well as initiate LEV development at lower angles of attack?

- If so, can a relationship between dynamic roughness properties (frequency of actuation and amplitude) and flow conditions (Reynolds number, airfoil pitching rate) be established?

- How does LEV development vary with airfoil pitching rate and Reynolds number?

- Are 2-D CFD simulations adequate enough to use as a tool to establish LEV "control" parameters based on dynamic roughness settings and flow characteristics?

- Is phase-averaged PIV analysis able to capture LEV development? 


\section{CHAPTER 2: EXPERIMENTAL ANALYSIS \\ 2.1 Model Design and Fabrication}

The NACA 0012 airfoil was chosen as the airfoil to study based on its wide use in previous research both at WVU and in the literature found in regards to dynamic stall. A chord length of 11.5 inches ( 0.2921 meters) was used to balance the desire to create a model as large as possible while keeping blockage constraints in mind. The motivation behind making the model as large as possible is to ease the fabrication and to increase the durability of the dynamic roughness sections. Although it is recommended to keep blockage below $10 \%$, at the highest angle of attack obtained during the pitching motion $\left(40^{\circ}\right)$ there is a blockage of $16 \%$. Although this is above the recommended value of $10 \%$, previous research indicates significant reductions in blockage effects when studying rapidly pitching airfoils [49].

The airfoil shape and support is provided by four aluminum ribs that were milled from half inch thick aluminum sheets on a numerically controlled Alliant end milling machine. This particular end mill has a resolution of 0.0002 inches. The profile for the airfoil was developed using the NACA four digit airfoil equation, which was then discretized into 141 points with a bias towards to leading edge. A plot of the points is shown in Figure 2.1.

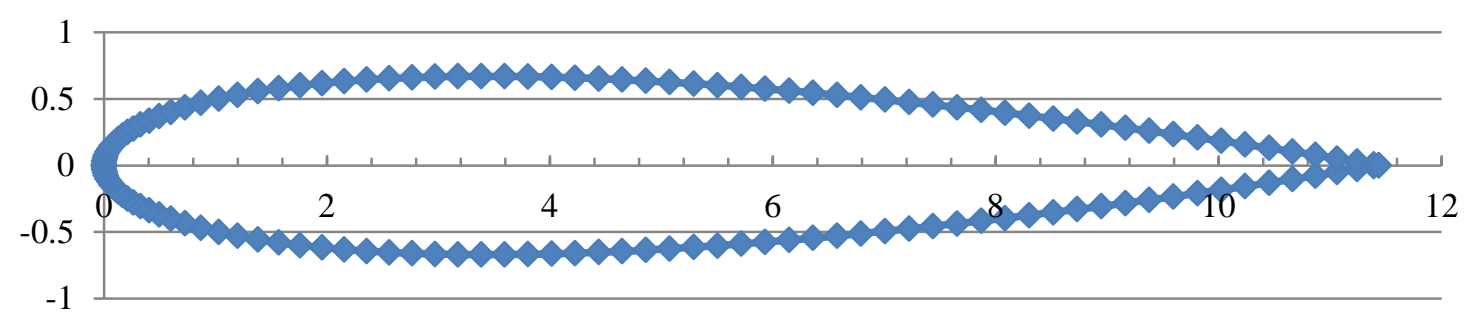

Figure 2.1 Points used to fabricate NACA 0012 rib.

Pockets were milled out of each rib to reduce weight and to allow spacing for appropriate plumbing connections for the dynamic roughness section of the leading edge. A 0.5 inch hole with a keyway was also milled at the quarter-chord position for shaft placement. Threaded screw holes were placed about every 1.25 inches $(0.11 \mathrm{c})$ to attach aluminum sheet metal skin. The screw holes were counter-sunk to create a smooth flush surface. Figure 2.2 provides a CAD drawing of the rib after milling and finishing. 


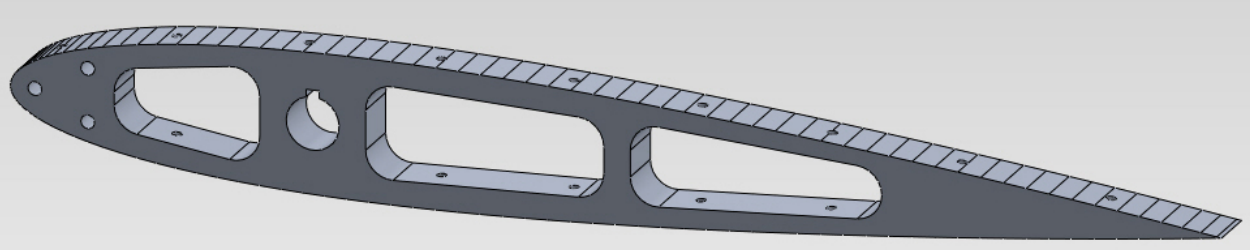

Figure 2.2 CAD representation of aluminum rib for NACA 0012 airfoil.

Between the two inner ribs is the location of the dynamic roughness section. The dynamic roughness section consists of two parts as shown in Figure 2.3. The leading edge part contains a cavity milled to create the dynamic roughness plenum as well as the individual dynamic roughness elements. Latex rubber is then glued over the entire section. This is then attached to the plumbing connection plate, which has an 1/8 inch NPT tapped hole to connect to the dynamic roughness actuation system. To mitigate air leakage a rubber o-ring is placed between the two sections when connected, see Figure 2.3. End plates are also attached at each span endpoint to eliminate wing-tip effects. The top end plate needed to be clear acrylic to permit an unobstructed camera view of the flow region of interest.
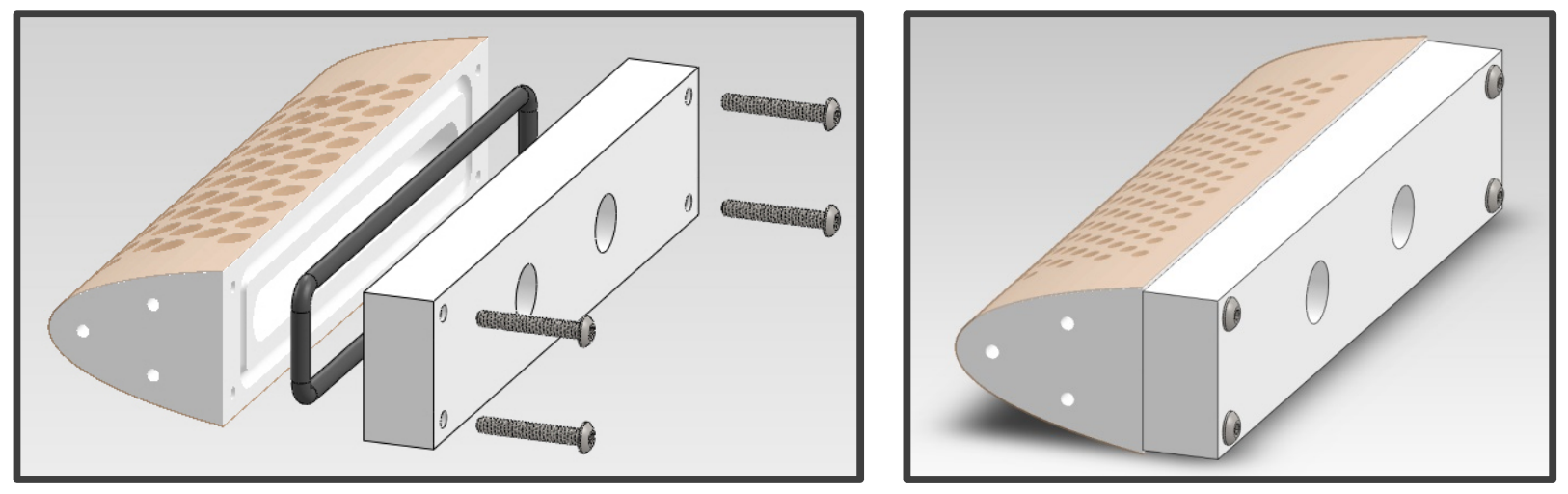

Figure 2.3 Exploded view and assembled view of dynamic roughness section (CAD representation), respectively.

Figure 2.4 provides a view of the model before the aluminum sheet metal skin is installed. Keyways in the shaft and ribs ensure accurate alignment and spacing as well as to prevent 
slippage while undergoing the oscillation during experimentation. One can also see how each threaded hole is counter-sunk into the rib to produce a smooth surface on the model.
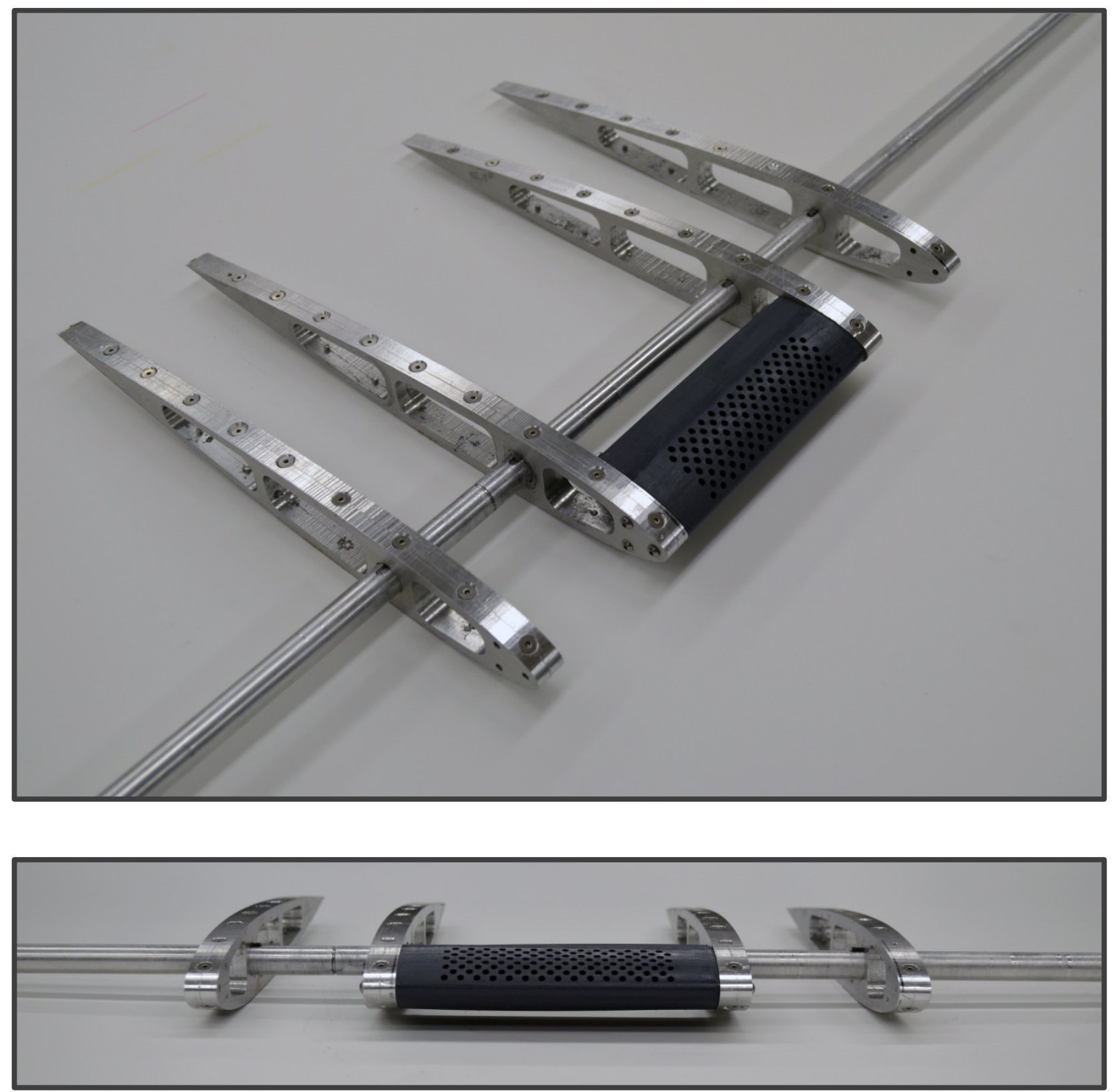

Figure 2.4 Assembly of NACA 0012 model with aluminum sheet metal skin removed. 


\subsection{Pitching Mechanism and Dynamic Roughness Actuation System}

To simulate a pitching airfoil a basic four bar linkage system was designed and fabricated to oscillate an airfoil between some minimum and maximum angle of attack. A $0.5 \mathrm{HP}$ variable speed DC motor drove the system, allowing the operator to vary the pitch rate. The minimum and maximum angle of attack can also be adjusted by changing the lengths of the linkages in the system. The assembled apparatus is shown in Figure 2.5

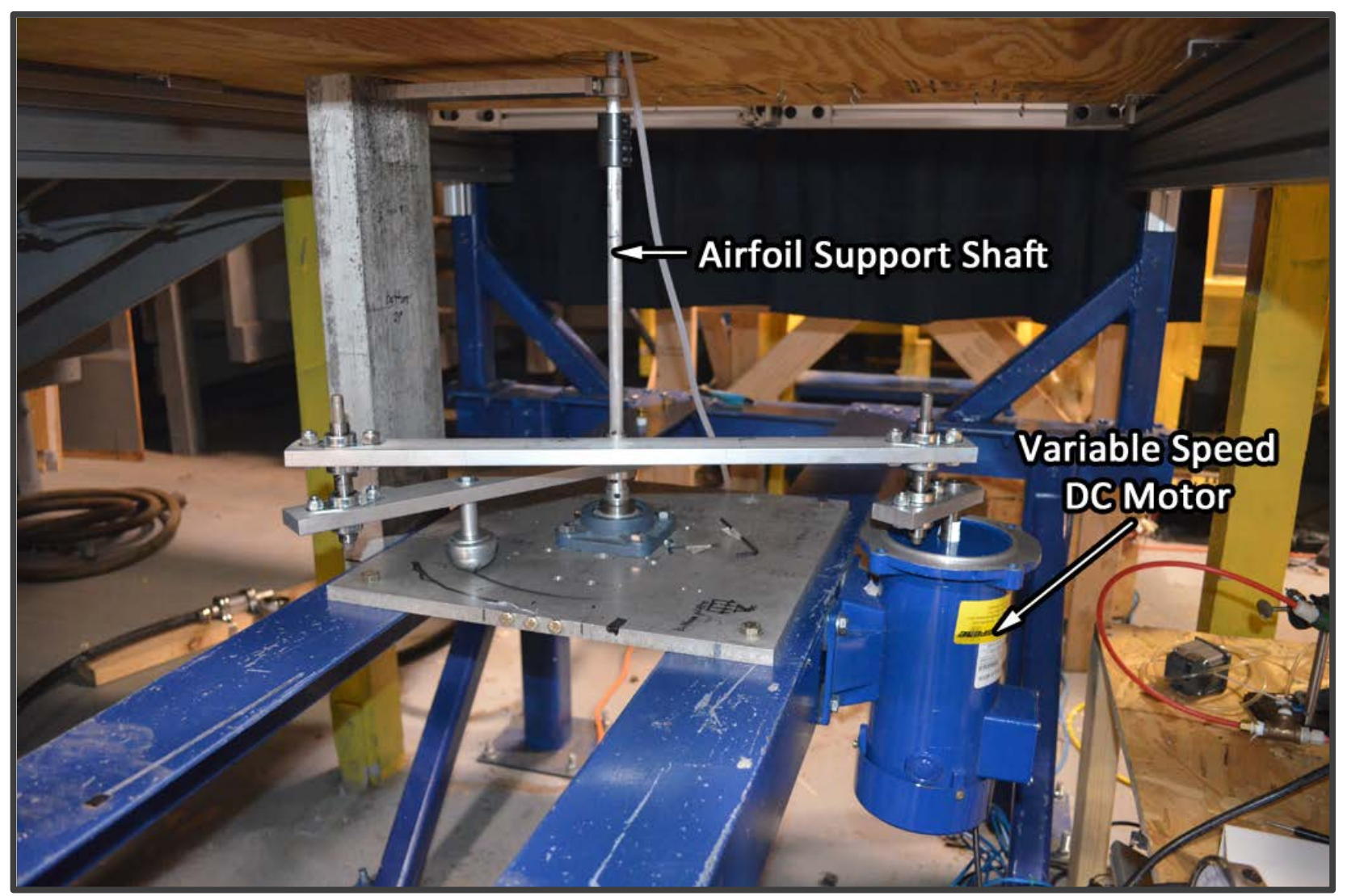

Figure 2.5 Mechanism designed and developed to oscillate an airfoil.

This method of pitching has been used extensively in previous research. By using such a system the instantaneous angle of attack, $\alpha$, can be represented by

$$
\alpha(t)=\alpha_{0}+\alpha_{1} \sin (\omega t)
$$


where $\alpha(t)$ is instantaneous angle of attack at time $t, \alpha_{0}$ is mean angle of attack, $\alpha_{1}$ is oscillation magnitude, and $\omega$ is circular frequency in $\mathrm{rad} / \mathrm{s}$. The circular frequency is often nondimensionalized using (2), and is termed reduced frequency, $k$.

$$
k=\frac{\omega c}{2 U_{\infty}}
$$

An oscillation frequency can be introduced whose cycle starts at a minimum angle of attack, pitches to a maximum angle of attack, and returns back to the minimum angle of attack. This oscillation frequency is measured in Hertz and can be related to the circular frequency, and thus the reduced frequency, by (3).

$$
f=\frac{\omega}{2 \pi}
$$

The motor is able to operate up to 120 RPM before the unbalanced nature of the mechanism causes significant vibration. This correlates to an oscillation frequency of $2 \mathrm{~Hz}$. This limitation is very suitable for the pitching frequencies desired to study. This is the only setup limitation since the maximum and minimum angle of attack can be altered by changing linkage lengths, as well as the mean angle of attack can be altered by adjust the initial shaft position.

To create dynamic roughness a pneumatic system was developed, as shown in Figure 2.6. A simple 27 cubic centimeter displacement two-stroke motor was connected via hard plastic tubing to the dynamic roughness plenum. A separate $0.5 \mathrm{HP}$ variable speed DC motor drove a beltpulley system that drove the motor, which in turn created an oscillating pressure source for the dynamic roughness plenum. This system allows the frequency of actuation to be accurately controlled. A shortcoming of this approach is the inability to control amplitude with any reasonable accuracy. A small valve is placed near the outlet of the two-stroke motor which can be opened to divert some mass flow, which in turn reduces amplitude. For the present work the valve remained shut to create the maximum possible amplitude, since during preliminary experimental analysis small amplitudes had no noticeable effect on the leading edge vortex. 


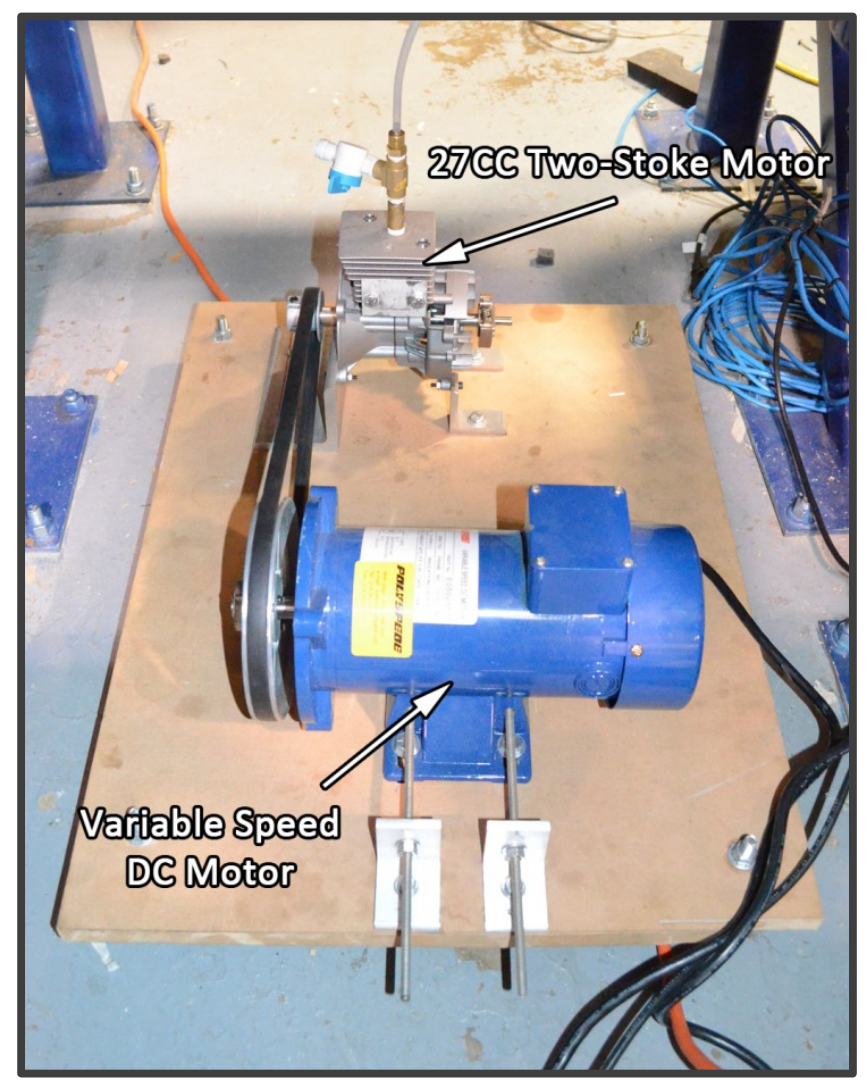

Figure 2.6 Dynamic roughness pneumatic actuation system.

The DC motor controller has a dial indicator spanning from 0 to 10 . Measurements were taken at each setting to develop a relationship between controller indication and frequency of actuation. The frequencies at which the roughness elements are actuated were measured three separate ways to confirm the displayed frequency. The first method used a non-contact tachometer connected to a digital numeric indicator. The digital readout was calibrated to display the frequency in units of cycles per minute with a resolution of 0.1 cycles per minute. The value given by the tachometer was confirmed by use of a stroboscope. It is important to mention that both of these methods measure the frequency of rotation of the two-stroke motor, not the actual dynamic roughness actuation. To confirm that the frequency of the two-stroke motor directly correlated to the frequency of actuation of the roughness elements high-speed video was used to capture the motion of the roughness elements. Video was recorded at a frame rate of 2000 frames per second. The high-speed video showed that the dynamic roughness actuation frequency can be measured via the frequency of the two-stroke motor. 
High-speed video was also used to calculate the amplitude of the dynamic roughness elements. This was accomplished by placing an object of known diameter in close proximity to the dynamic roughness elements during recording. This allowed for a conversion factor to be created between the pixel length on the recording with a physical unit of length. The amplitude of the dynamic roughness elements while being actuated increased with an increase in frequency. At a controller setting of $3(20 \mathrm{~Hz})$ the amplitude was measure to be $0.205 \mathrm{~mm}$, and at a setting of $10(89 \mathrm{~Hz})$ the amplitude was $0.301 \mathrm{~mm}$. The relationship between controller setting and roughness amplitude varied linearly. There was also a difference in measured amplitude based on whether the two-stroke motor was initiated from bottom dead center (so the mass flow rate from the two-stroke motor would initially create a positive pressure) or from top dead center (created an initial suction pressure in the dynamic roughness plenum) in some instances. When a difference did occur, the amplitude was always less when the motor was started from top dead center. The variation in amplitude between piston initial position was less than 5 percent. Even though this is such a small percentage of the amplitude, care was taken to start the dynamic roughness apparatus with the piston initially in the bottom dead center position.

\subsection{Instrumentation and Experimental Setup}

For experimental analysis Particle Image Velocimetry (PIV) data was collected. Both stereo and planar PIV setups were used, with stereo PIV having the ability to provide all three components of velocity. A basic description of the PIV principle is given as follows. PIV operates by recording two images of particles illuminated by a laser with a short time delay between each image. During the short time interval the particles have moved by a certain displacement. The velocity is simply the change in displacement divided by the time interval. The PIV system used for this research was a commercially purchased system developed by LaVision, Inc. The system consists of a two Nd:Yag lasers that emit light at a wavelength of $532 \mathrm{~nm}$. A laser arm consisting of finely tuned mirrors is connected to the laser output to allow easy manipulation of the laser output position. Attached to the laser output is a cylinder optic that spreads the laser from a beam to a sheet or plane. Two ImagerProX CCD cameras are used to capture the images and an aerosol seeder generates uniform particles with $1 \mu \mathrm{m}$ diameter to seed the flow. The cameras capture images at a resolution of 4008 x 2672 pixels (11 megapixel) at a maximum frame rate of 5 fps. A programmable timing unit (PTU) and the DAVIS software provided by 
LaVision, Inc. controls the synchronization between laser illumination and image capture by the cameras. Figure 2.7 provides a labeled overview of PIV setup with respect to the wind tunnel section. One can see that for a vertically mounted model the cameras are placed on top of the test section while the laser sheet is emitted from the side of the test section. Care must be taken to provide the clearest view for both the cameras and laser sheet.

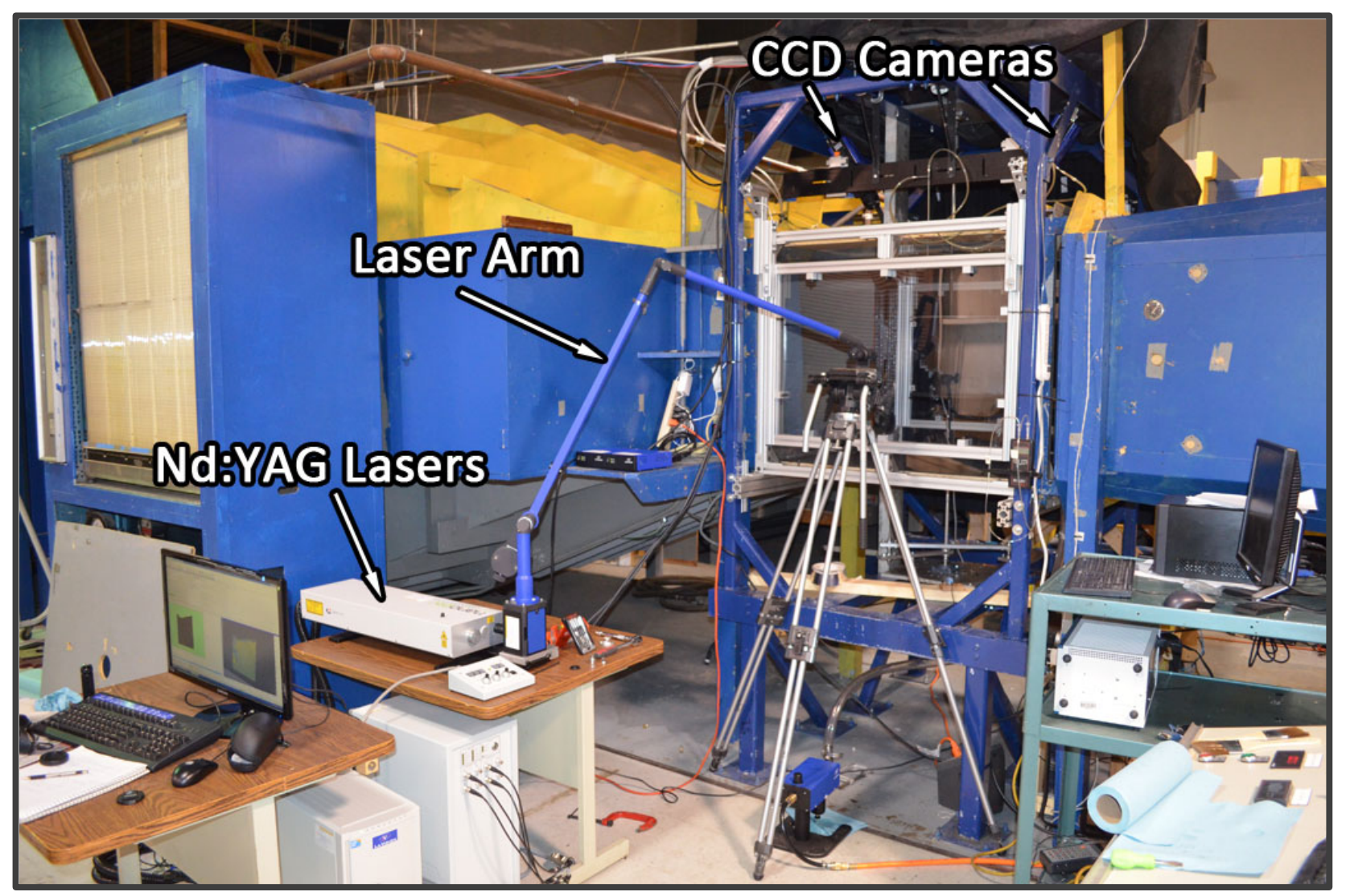

Figure 2.7 Labeled image of PIV setup, highlighting positioning of cameras and laser output with respect of wind tunnel test section.

The experimental setup is as follows, the model is mounted vertically in the WVU subsonic closed circuit wind tunnel with the quarter chord position in the horizontal center of the test section. The mid-span location is positioned in the vertical center of the test section as shown in Figure 2.8. The pitching mechanism is mounted below the test section and attaches to a shaft that runs vertically through the test section and model at the quarter-chord location. This shaft is supported in four positions which are dictated by the wind tunnel test section geometry as well as a steel support structure that surrounds the test section, but is isolated from the test section itself. 
This is to ensure that no vibrations emanating from the wind tunnel itself can be transmitted to the model or instrumentation. The four locations are described as follows, (1) at the base near where the pitching mechanism linkage arm is connected, (2) just under and outside the wind tunnel test section, (3) just above and outside the test section, and (4) at the top of the steel support structure where an absolute encoder is attached to the shaft to indicate angle of attack.

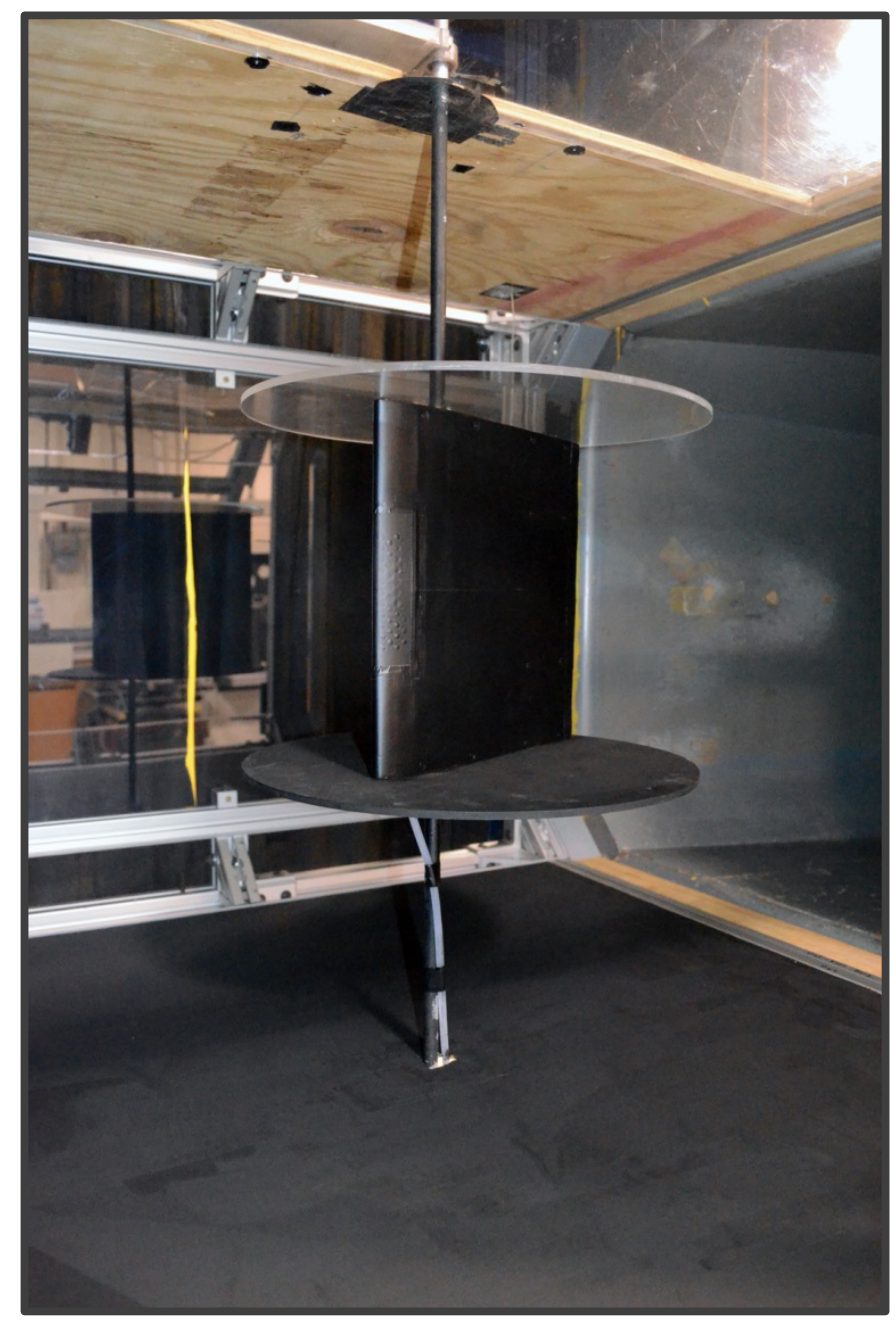

Figure 2.8 Mounting position of the NACA 0012 model in the WVU subsonic closed circuit wind tunnel.

Certain considerations have to be taken into account when setting up the experiment for PIV data collection. There has to be clear view of the area of interest both for the laser illuminated plane as well as the cameras. The test section of the wind tunnel was developed with clear acrylic windows on either side of the test section, allowing unimpeded laser illumination. The cameras were mounted near the top of the test section so as to be perpendicular to the plane illuminated 
by the laser. The "roof" of the test section was modified by embedding a clear acrylic section to permit a clear view for the cameras to view the airfoil and laser plane. As mentioned above, the top end plate of the model was also made of clear acrylic to allow full view by the PIV camera system. The end of the laser arm is firmly attached to a camera tripod allowing easy manipulation of the laser plane.

\subsection{PIV Results}

The PIV analysis can be broken down into three groups that can be posed as questions:

- How does the LEV development change with respect to Reynolds number?

- How does the LEV development change with respect to reduced oscillation frequency?

- Can dynamic roughness alter the development of the LEV?

To evaluate how the LEV development changes with respect to Reynolds number PIV data was collected at three different Reynolds number: 80,000; 150,000; and 200,000. The reduced oscillation frequency was held constant at $k=0.1$. It is important to recall (3) to understand that the reduced oscillation frequency is non-dimensionalized with respect to free-stream velocity, $U_{\infty}$, and airfoil chord length, $c$. Thus, the dimensional pitch rate is different among each case. Table 2.1 briefly outlines the dimensional characteristics for each case. As is typical in PIV data collection, a series of images were averaged to provide an average flow field at each angle of attack desired. This was accomplished by triggering the cameras and lasers based on the output of the absolute encoder embedded on top of the model support shaft. A LabView program was developed to trigger the system at any desired angle of attack. This allowed for a phase averaged view at each angle of attack. 100 time instances were averaged at each angle of attack for PIV analysis. 
Table 2.1 Conditions used to observe changes in LEV development with respect to Re.

\begin{tabular}{|c|c|c|c|}
\hline Re & $\begin{array}{c}\mathbf{U}_{\infty} \\
(\mathrm{m} / \mathrm{s})\end{array}$ & $\begin{array}{c}\omega \\
(\mathrm{rad} / \mathrm{s})\end{array}$ & Motor RPM \\
\hline 80,000 & 4.0 & 2.74 & 26.2 \\
\hline 150,000 & 7.5 & 5.14 & 49 \\
\hline 200,000 & 10.0 & 6.85 & 65.4 \\
\hline
\end{tabular}

The results of the Reynolds number dependence study are summarized in Figure 2.9. Contours of the chord-wise velocity component overlaid with the velocity vector and streamlines are plotted at three different angles of attack at each Reynolds number. Please note, the PIV results collected at a Reynolds number of 150,000 were taken at a different time and with a slightly different camera angle than the results gathered for the other two cases. This information is to let the reader understand why the angle of the airfoil in the $\mathrm{Re}=150,000$ cases appears different than the other two instances. 

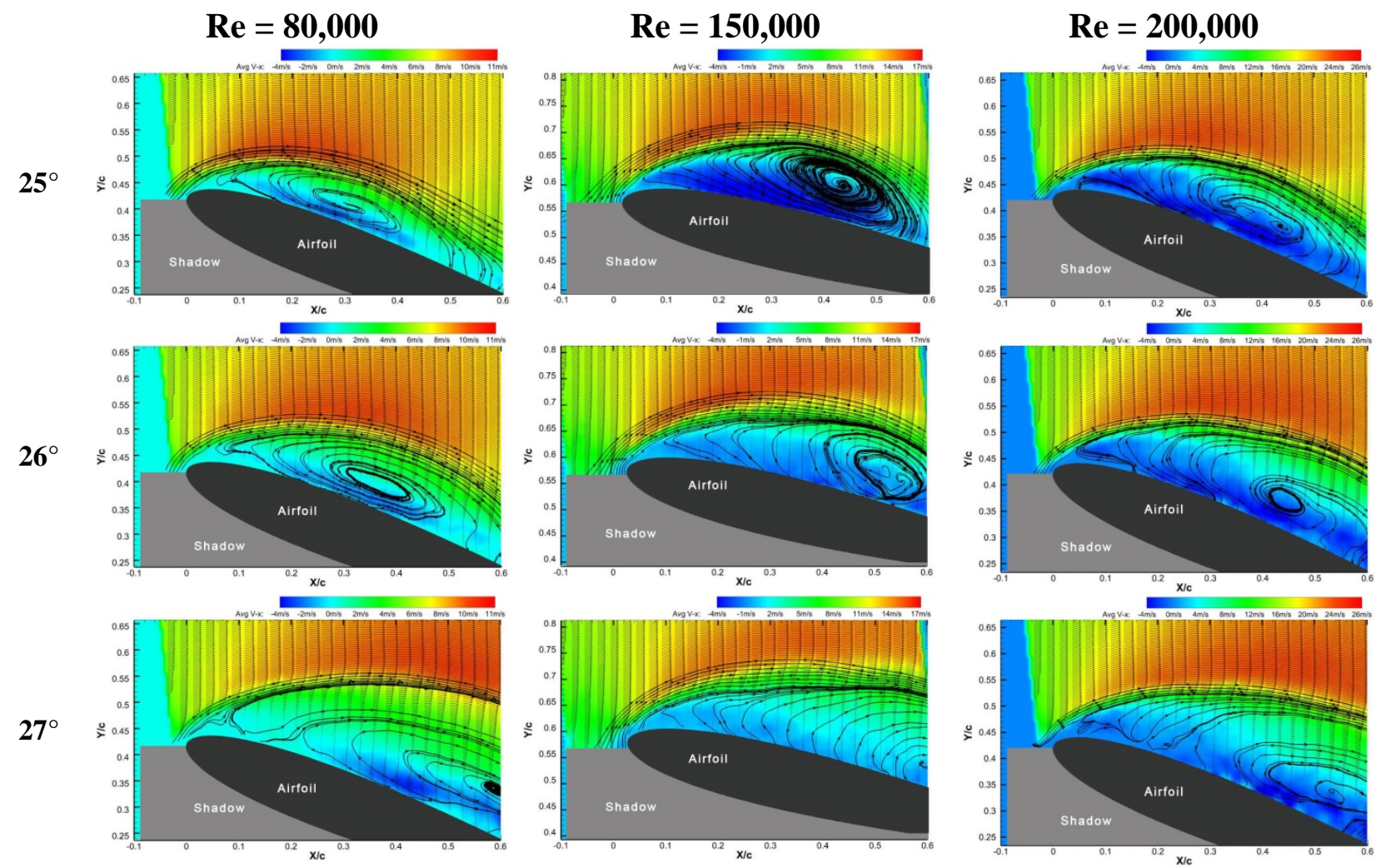

Figure 2.9 Planar PIV results of the evolution of the LEV at varying Reynolds numbers at the same reduced frequency, $k=0.1$.

By examining the results shown in Figure 2.9 one can see there is relatively little variation from one Reynolds number to the next. Although there is some discussion as to the dependence on Reynolds numbers by previous researchers [25], the majority conclude that Reynolds number only plays a minor role in dynamic stall [21] [33]. The results shown here tend to confirm the latter. A subtle difference between Reynolds numbers appears to be a small displacement downstream of the major circulation region as Reynolds number is increased.

A similar study was done to investigate the effects of increasing the reduced frequency, and in essence the pitching rate. In this case the Reynolds number was held constant at $\operatorname{Re}=80,000$ while PIV data was recorded at $k=0.1, k=0.15$, and $k=0.2$. In contrast to Reynolds number dependence it is evident reduced frequency plays an important role in LEV development. With an increase in reduced frequency LEV development is delayed until higher angles of attack. This acts to retain lift to higher angles of attack as well as delay the onset of the sudden stall and abrupt change in pitching moment associated with the LEV being convected downstream. The results are provided in Figure 2.10 

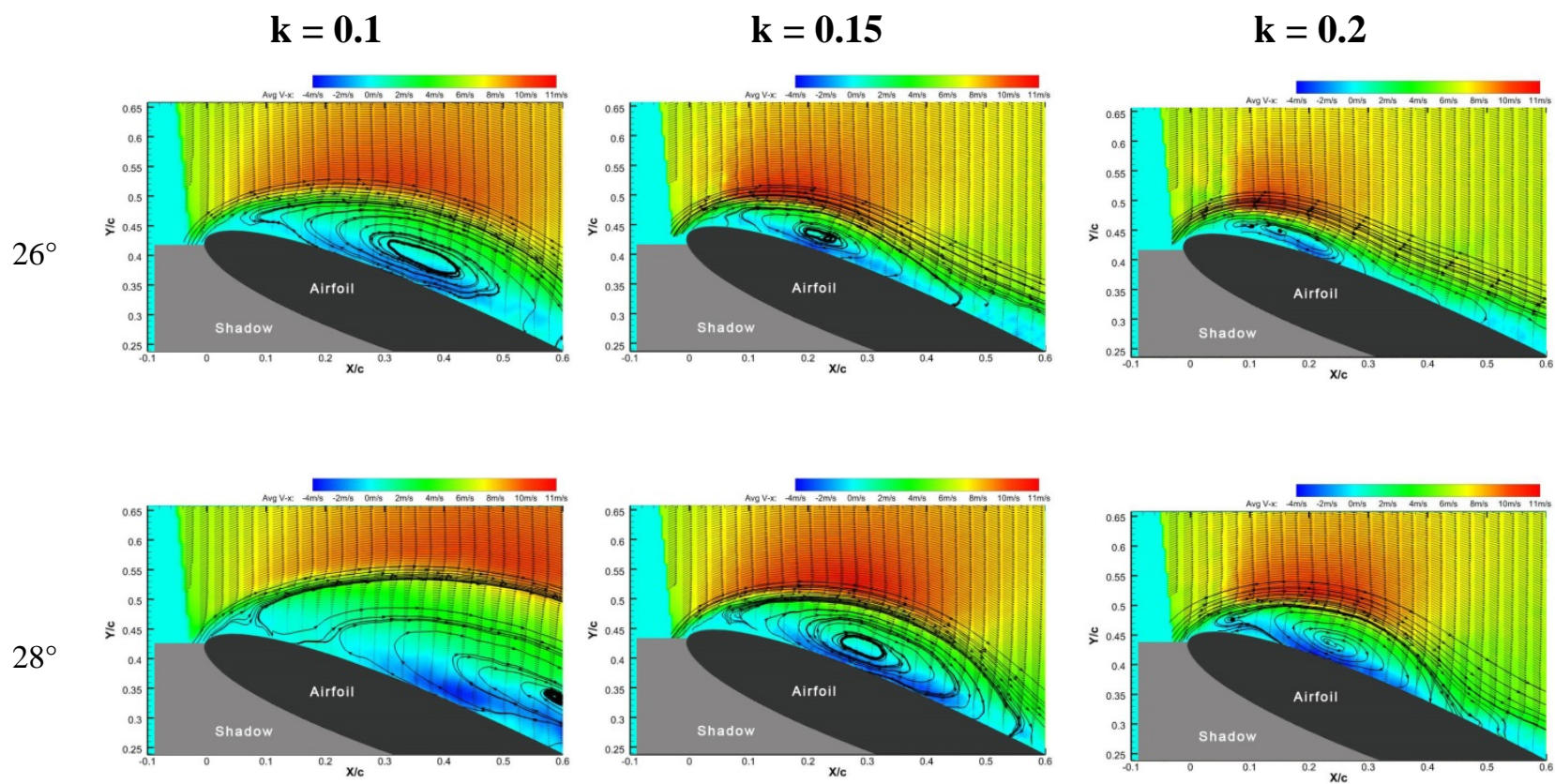

Figure 2.10 Planar PIV results of the change in LEV formation as reduced frequency, $\mathrm{k}$, is increased and Reynolds number is held constant at $\mathbf{8 0 , 0 0 0}$.

To evaluate the effectiveness of dynamic roughness in altering the LEV, studies were conducted at a variety of flow conditions and dynamic roughness actuation frequencies. At most conditions LEV development could not be altered in any significant manner. Most differences observed were minor changes in recirculating region distance from the airfoil surface. However, eventually a condition was found to show noticeable change in LEV development. Figure 2.11 displays a condition in which dynamic roughness was able to delay initial formation of the LEV as well as the growth of the LEV. The Reynolds number was 150,000 and the dynamic roughness required actuation at the highest frequency that could be obtained using the current actuation device, which was $89 \mathrm{~Hz}$. 

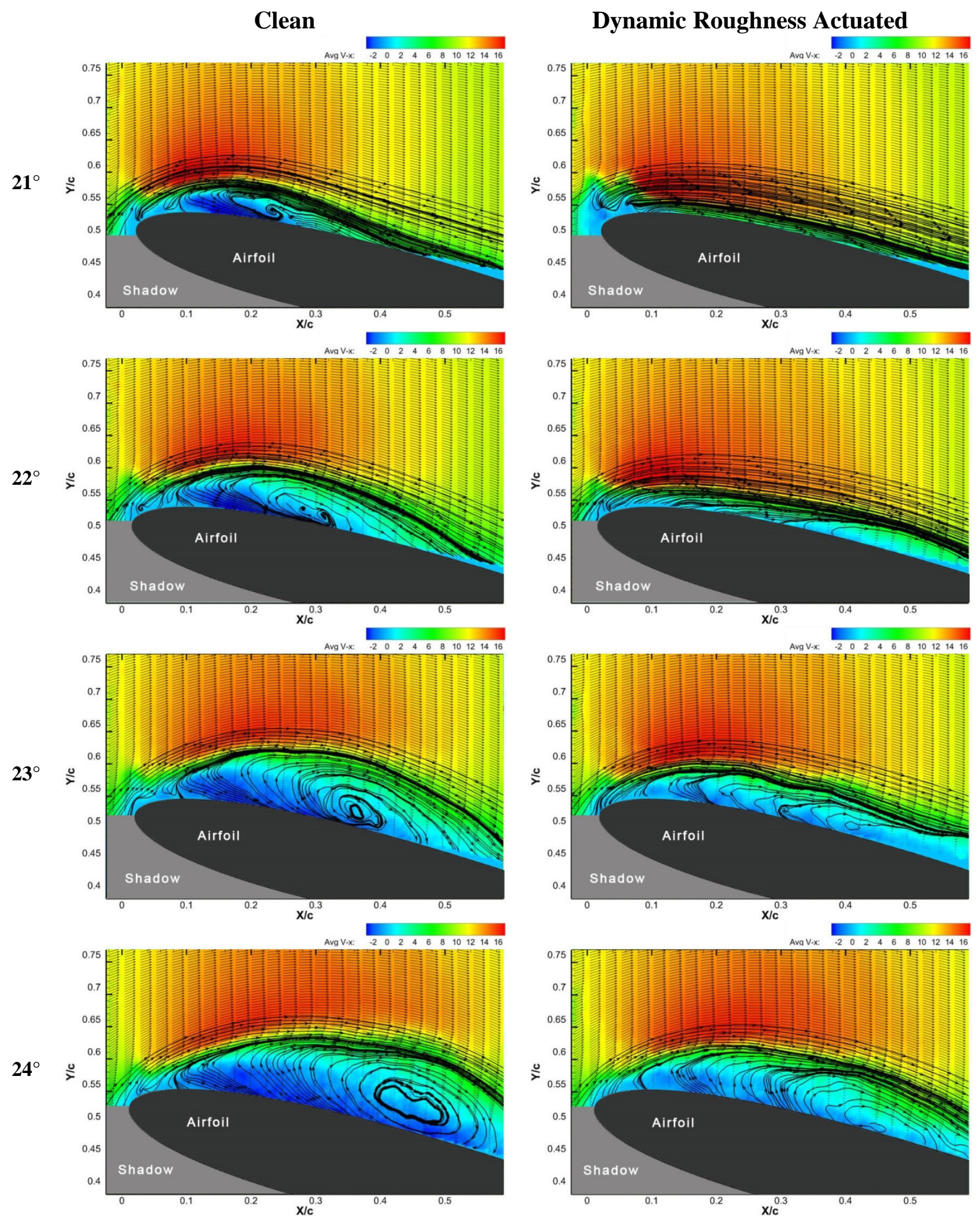

Figure 2.11 Contours of chord-wise velocity magnitude overlaid with velocity vectors and streamlines at $R e=150,000$ and $k=0.1$. At this flow condition dynamic roughness was able to delay the development of the LEV. 
Once this condition was found the same dynamic roughness actuation frequency was tested at other flow conditions. If Reynolds number was reduced or the reduced frequency was changed LEV delay was lost. An example of such a case is shown in Figure 2.12. At a Reynolds number of 80,000 and a reduced frequency of 0.1 dynamic roughness was not able to make any noticeable differences in LEV development.
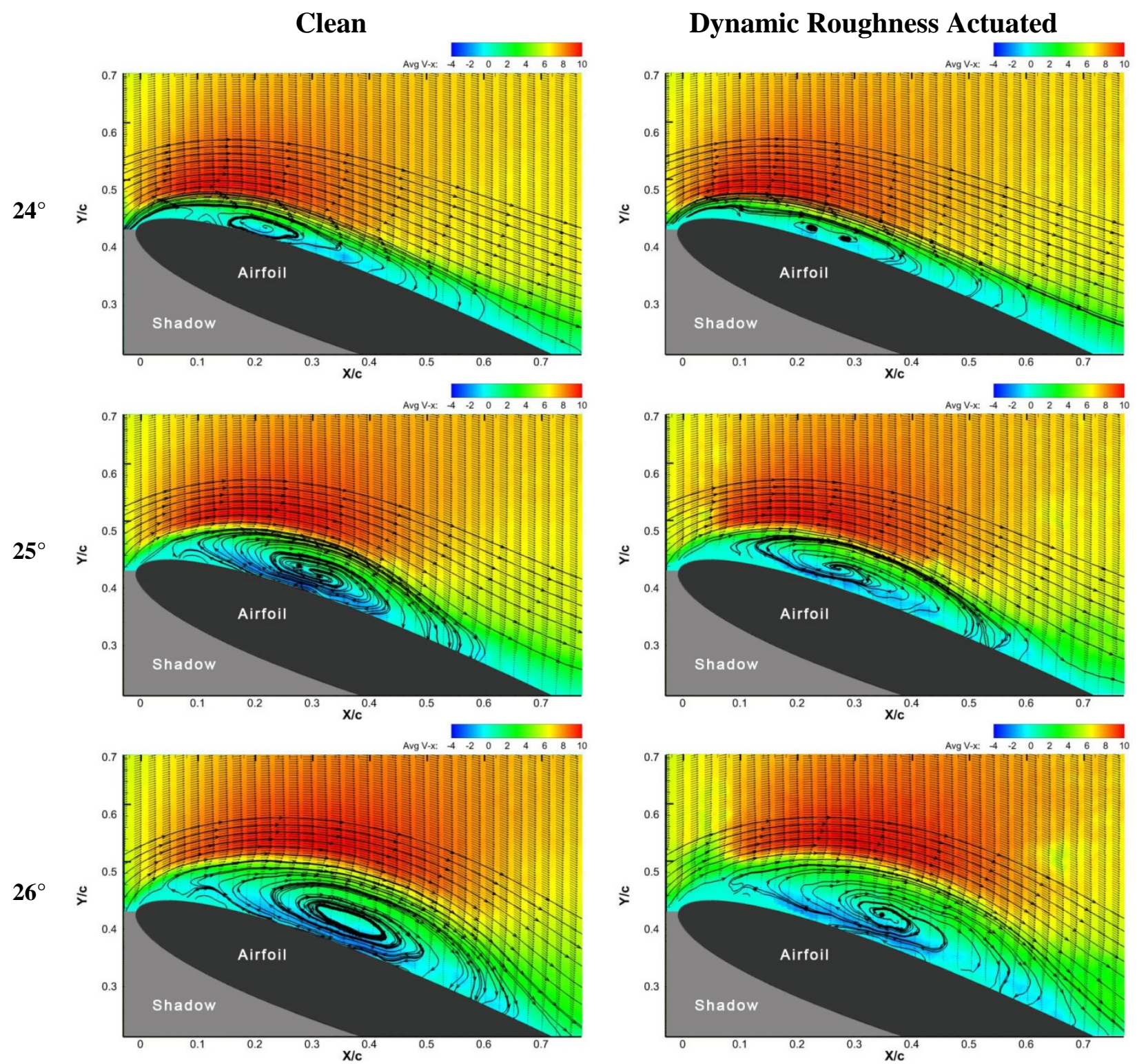

Figure 2.12 Contours of chord-wise velocity magnitude overlaid with velocity vectors and streamlines at $\mathrm{Re}=\mathbf{8 0 , 0 0 0}$ and $\mathrm{k}=\mathbf{0 . 1}$. At this flow condition dynamic roughness did not have any noticeable effect on LEV development. 


\subsection{Stereoscopic and Planar PIV Discussion}

All results presented above have been planar (two-dimensional) in nature. As stated, both planar and stereoscopic PIV data were collected. A brief discussion on any differences in results is offered. To analyze differences one can look at the case in which dynamic roughness had a noticeable effect on LEV development. Presented in Figure 2.13 is a comparison between the planar results with the stereoscopic results.

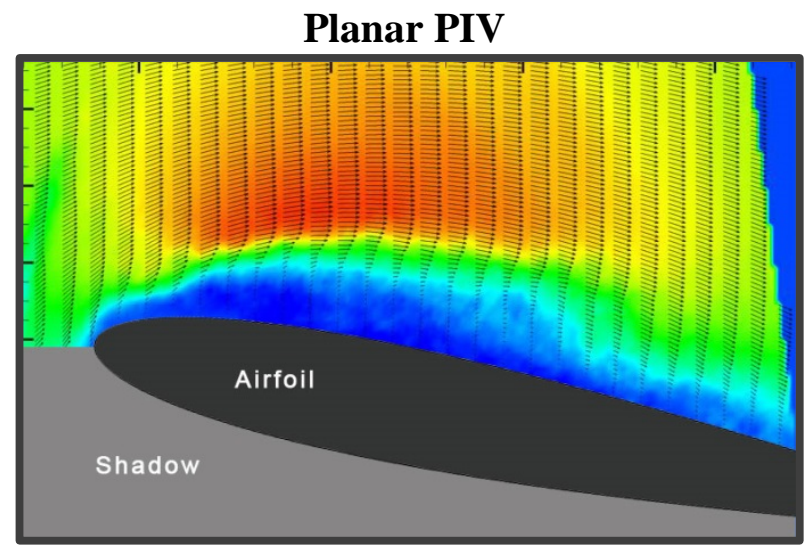

(a)

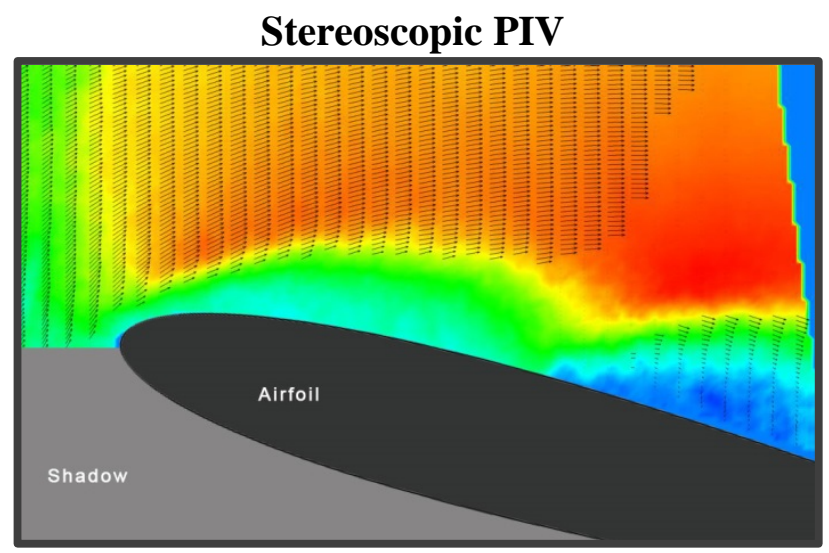

(b)

Figure 2.13 Chord-wise velocity contours with velocity vectors for the same flow condition with collected with (a) planar PIV and (b) stereoscopic PIV.

As shown in the figure, what appears to be a secondary recirculation region is captured by the stereoscopic PIV, but neglected by the planar PIV. In addition, in the planar PIV data the flow clearly seems to be recirculating, in the same region on the stereoscopic results the flow does not necessarily appear to be recirculating, but merely at a much slower magnitude in the chord-wise component. What appears to be missing vectors in that same region as well as the region above the secondary recirculation region are actually vectors that have a large span-wise component. If one skews the view to one side or the other, the velocity component in the span-wise direction becomes more apparent.

Figure 2.14 highlights the span-wise component of velocity by displaying contours of the velocity in the span-wise direction. One can clearly from this image the high velocity in the span-wise direction in the area of recirculation. 


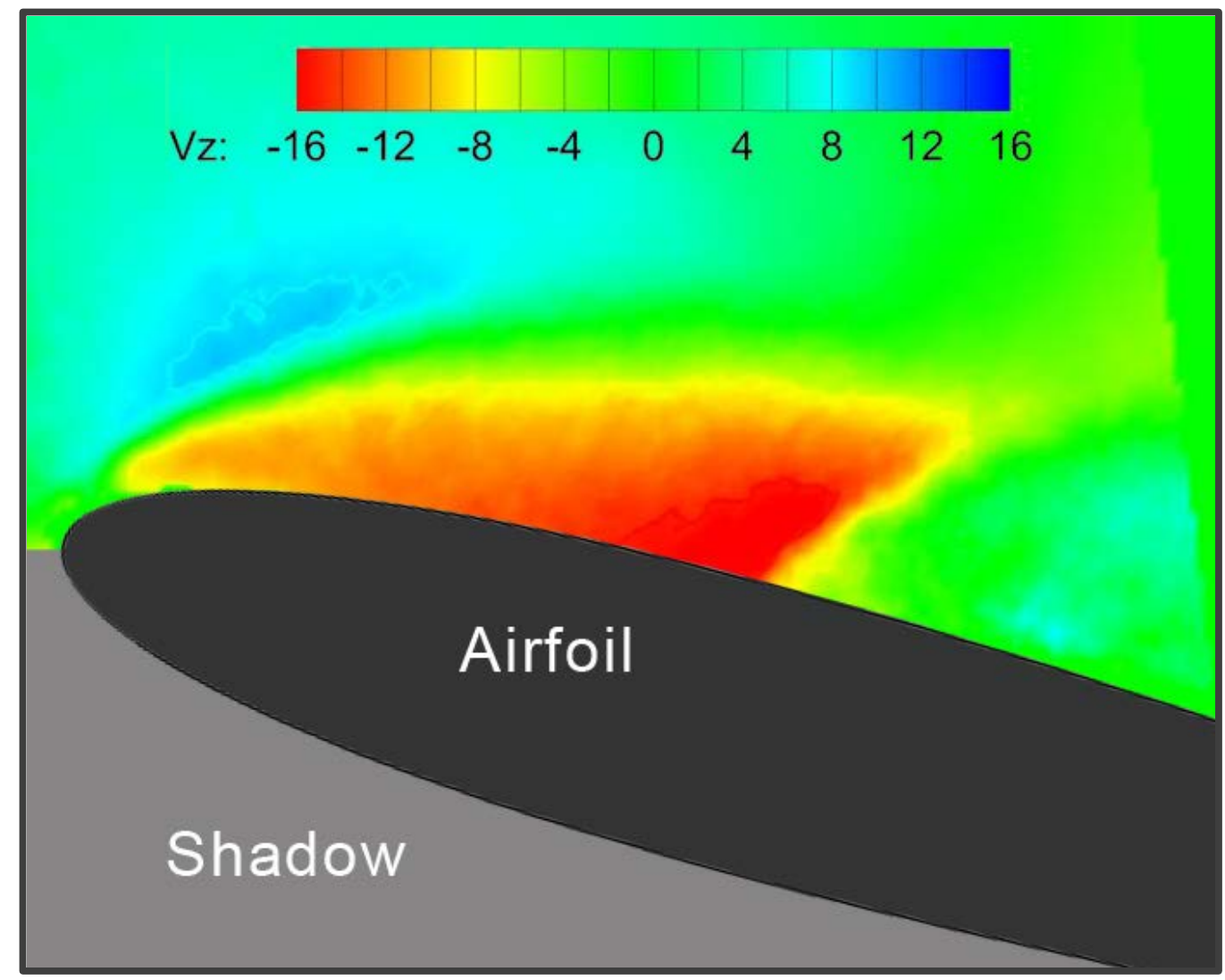

Figure 2.14 Contours of span-wise velocity from stereoscopic PIV analysis.

It is not entirely clear whether the span-wise flow is an artifact of the PIV correlation process, or indeed present. Three-dimensional computational simulations do show a trend toward random span-wise flow, but the PIV data suggest there is a bias to flow in a certain direction whether you are looking within the recirculation region or beyond it. This could be due to a slight span-wise component in the free-stream velocity of the wind tunnel that has been documented by previous experiments. This span-wise flow has even been recorded as changing severity with increase test section speed.

\subsection{PIV Uncertainty Analysis}

Although there is no universally accepted procedure to performing an uncertainty analysis when conducting PIV experiments, an attempt was made to find an approach that provides, at the very least, a general idea of the uncertainties associated with PIV analysis. A recommended procedure was found that was developed by the International Towing Tank Conference that was very in depth in its scope of possible sources of uncertainty in PIV measurements [50]. The 
sources of error are vast and the reader is encourage to view the referenced document for a clear understanding of each source. As an example, typical sources of error are shown in Table 2.2.

Typical sources of error for PIV measurement.

\begin{tabular}{|l|l|l|}
\hline \multicolumn{1}{|c|}{ Calibration } & Digital Image Capture & \multicolumn{1}{|c|}{ Data Analysis } \\
\hline - Illumination uniformity & - Background noise & - Correlation calculations \\
- CCD distortion & - Light scattering & - Particle traceability \\
- Lens distortion & - Fluctuation of laser & - Particle uniformity \\
- Refraction at window & power & - Vector averaging \\
- Physical position & - CCD distortion & \\
& - Sampling frequency & \\
& - Pulse timing & \\
\hline
\end{tabular}

The principal dimensions of target measurement consist of the following sub-systems: (1) Calibration, (2) Flow Visualization, (3) Image Detection, and (4) Data Processing, and are listed in Table 2.3. Each of these sub-systems is then broken down to see how their associated error sources effects the three basic measurements of velocity, position, and time. The uncertainties for these measurements are provided in Table 2.4, Table 2.5, and Table 2.6, respectively. The process of uncertainty analysis involves estimating the error for each possible source, then applying a sensitivity calculation to that error. Then for each uncertainty type (velocity, position, and time) the uncertainties are root sum squared to provide a total uncertainty. As shown in the following tables, the total uncertainty in $x$-velocity is $0.35 \mathrm{~m} / \mathrm{s}$, the total uncertainty for position is $1.09 \mathrm{~mm}$, and the total uncertainty for time is $1.00 \times 10^{-8} \mathrm{~s}$. 
Table 2.3 Principal dimensions of PIV measurement.

\begin{tabular}{|c|c|}
\hline \multicolumn{2}{|c|}{ Target Flow of Measurement } \\
\hline Target Flow & 2-D Air Flow \\
\hline Measurement Facility & Closed-Loop Wind Tunnel \\
\hline Measurement Area & $460 \times 290 \mathrm{~mm}^{2}$ \\
\hline Uniform Flow Speed & $10 \mathrm{~m} / \mathrm{s}$ \\
\hline \multicolumn{2}{|c|}{ Calibration } \\
\hline Distance of Reference Points & $15 \mathrm{~mm}$ \\
\hline Distance of Reference Image & 150.64 pixels \\
\hline Magnification Factor & $0.099575146 \mathrm{~mm} /$ pixel \\
\hline \multicolumn{2}{|c|}{ Flow Visualization } \\
\hline \multicolumn{2}{|l|}{ Tracer Particle } \\
\hline Average Diameter & $0.001 \mathrm{~mm}$ \\
\hline Standard Deviation of Diameter & $0.0001 \mathrm{~mm}$ \\
\hline Average Specific Gravity & 0.914 \\
\hline Light Source & Double Pulse Nd:YAG laser \\
\hline Thickness of Laser Light Sheet & $1 \mathrm{~mm}$ \\
\hline Time Interval & $60 \mu \mathrm{s}$ \\
\hline \multicolumn{2}{|c|}{ Image Detection } \\
\hline \multicolumn{2}{|l|}{ Camera } \\
\hline Spatial Resolution & $4008 \times 2672$ pixels \\
\hline Sampling Frequency & $5 \mathrm{fps}$ \\
\hline Gray Scale Resolution & 14 bit \\
\hline Cell Size & $9 \times 9 \mu \mathrm{m}^{2}$ \\
\hline \multicolumn{2}{|l|}{ Optical System } \\
\hline Distance from the Target & $721.006 \mathrm{~mm}$ \\
\hline Length of Focus & $62.1126 \mathrm{~mm}$ \\
\hline F Number of Lens & 2.8 \\
\hline \multicolumn{2}{|c|}{ Data Processing } \\
\hline Pixel Unit Analysis & Cross correlation method \\
\hline Correlation Area Size & $16 \times 16$ pixels \\
\hline
\end{tabular}


Table 2.4 Summary of uncertainties for velocity.

\begin{tabular}{|c|c|c|c|c|c|c|c|c|}
\hline Parameter & Category & Error Sources & $u\left(x_{i}\right)$ & (unit) & $c_{i}$ & (unit) & $c_{i} u\left(x_{i}\right)$ & $u_{c}$ \\
\hline \multirow{5}{*}{$\begin{array}{c}\alpha \\
(\mathrm{mm} / \mathrm{pix})\end{array}$} & \multirow{5}{*}{ Calibration } & Reference Image & 0.7 & (pix) & $6.61 \mathrm{E}-04$ & $\left(\mathrm{~mm} / \mathrm{pix}^{2}\right)$ & 4.63E-04 & \multirow[b]{5}{*}{ 7.06E-04 } \\
\hline & & Physical Distance & 0.02 & $(\mathrm{~mm})$ & $6.64 \mathrm{E}-03$ & $(1 /$ pix $)$ & $1.33 \mathrm{E}-04$ & \\
\hline & & Image Distortion by Lens & 0.753 & (pix) & $6.61 \mathrm{E}-04$ & $\left(\mathrm{~mm} / \mathrm{pix}^{2}\right)$ & $4.98 \mathrm{E}-04$ & \\
\hline & & Board Position & 0.5 & $(\mathrm{~mm})$ & $1.38 \mathrm{E}-04$ & $(1 /$ pix $)$ & $6.91 \mathrm{E}-05$ & \\
\hline & & Parallel Board & 0.035 & (rad) & $3.49 \mathrm{E}-03$ & (mm/pix) & $1.22 \mathrm{E}-04$ & \\
\hline \multirow{5}{*}{$\begin{array}{c}\Delta \mathrm{X} \\
\text { (pix) }\end{array}$} & \multirow{3}{*}{ Acquisition } & Laser Power Fluctuation & 0.0014 & $(\mathrm{~mm})$ & $1.00 \mathrm{E}+01$ & $(\mathrm{pix} / \mathrm{mm})$ & $1.41 \mathrm{E}-02$ & \multirow[b]{5}{*}{ 2.03E-01 } \\
\hline & & Image Distortion by CCD & & & & & $0.00 \mathrm{E}+00$ & \\
\hline & & Normal View Angle & 0.035 & (rad) & $3.49 \mathrm{E}-03$ & (mm/pix) & $1.22 \mathrm{E}-04$ & \\
\hline & \multirow{2}{*}{ Reduction } & Mis-matching Error & 0.2 & (pix) & $1.00 \mathrm{E}+00$ & & $2.00 \mathrm{E}-01$ & \\
\hline & & Sub-pixel Analysis & 0.03 & (pix) & $1.00 \mathrm{E}+00$ & & $3.00 \mathrm{E}-02$ & \\
\hline$\Delta \mathrm{t}(\mathrm{s})$ & Acquisition & Timing & $1.00 \mathrm{E}-08$ & $(s)$ & $1.00 \mathrm{E}+00$ & & $1.00 \mathrm{E}-08$ & $1.00 \mathrm{E}-08$ \\
\hline \multirow{2}{*}{$\delta \mathrm{u}(\mathrm{mm} / \mathrm{s})$} & \multirow{2}{*}{ Experiment } & Particle Trajectory & 1 & $(\mathrm{~mm} / \mathrm{s})$ & $1.00 \mathrm{E}+00$ & & $1.00 \mathrm{E}+00$ & \multirow[b]{2}{*}{$3.30 \mathrm{E}+01$} \\
\hline & & 3-D Effects & 33.03 & $(\mathrm{~mm} / \mathrm{s})$ & $1.00 \mathrm{E}+00$ & & $3.30 \mathrm{E}+01$ & \\
\hline \multicolumn{9}{|c|}{ Summary } \\
\hline$\alpha$ & & Magnification Factor & 7.06E-04 & (mm/pix) & $1.00 \mathrm{E}+05$ & (pix/s) & $7.10 \mathrm{E}+01$ & \\
\hline$\Delta \mathrm{X}$ & & Image Displacement & 2.03E-01 & (pix) & $1.66 \mathrm{E}+03$ & $(\mathrm{~mm} / \mathrm{pix} / \mathrm{s})$ & $3.36 \mathrm{E}+02$ & \\
\hline$\Delta \mathrm{t}$ & & Image Interval & $1.00 \mathrm{E}-08$ & $(s)$ & $1.67 \mathrm{E}+08$ & $\left(\mathrm{~mm} / \mathrm{s}^{2}\right)$ & $1.67 \mathrm{E}+00$ & \\
\hline$\delta u$ & & Experiment & $3.30 \mathrm{E}+01$ & $(\mathrm{~mm} / \mathrm{s})$ & $1.00 \mathrm{E}+00$ & & $3.30 \mathrm{E}+01$ & \\
\hline \multicolumn{7}{|c|}{ Combined Uncertainty } & $u_{u}$ & $0.35(\mathrm{~m} / \mathrm{s})$ \\
\hline
\end{tabular}




\begin{tabular}{|c|c|c|c|c|c|c|c|c|}
\hline Parameter & Category & Error Sources & $u\left(x_{i}\right)$ & (unit) & $c_{i}$ & (unit) & $c_{i} u\left(x_{i}\right)$ & $u_{c}$ \\
\hline \multirow{2}{*}{$x_{s}, x_{e}$} & \multirow{2}{*}{ Acquisition } & Digital Error & 0.5 & (pix) & $9.96 \mathrm{E}-02$ & (mm/pix) & $4.98 \mathrm{E}-02$ & \\
\hline & & Non-uniformity of distribution & 4 & (pix) & $9.96 \mathrm{E}-02$ & (mm/pix) & $3.98 \mathrm{E}-01$ & \\
\hline$x_{0}$ & Calibration & Origin Correlation & 2 & (pix) & $9.96 \mathrm{E}-02$ & (mm/pix) & 1.99E-01 & \\
\hline$\alpha$ & & Magnification Factor & $4.98 \mathrm{E}-04$ & $(\mathrm{~mm} / \mathrm{pix})$ & 2004 & (pix) & $9.98 \mathrm{E}-01$ & \\
\hline \multicolumn{7}{|c|}{ Combined Uncertainty } & $u_{x}$ & $1.09(\mathrm{~mm})$ \\
\hline
\end{tabular}

Table 2.6 Summary of uncertainty for time.

\begin{tabular}{|c|c|c|c|c|c|c|c|c|}
\hline Parameter & Category & Error Sources & $u\left(x_{i}\right)$ & (unit) & $c_{i}$ & $($ unit) & $c_{i} u\left(x_{i}\right)$ & $u_{c}$ \\
\hline$t_{s}, t_{e}$ & Acquisition & Delay Generator/Pulse Time & $1.00 \mathrm{E}-08$ & $(\mathrm{~s})$ & $1.00 \mathrm{E}+00$ & & $1.00 \mathrm{E}-08$ & \\
\hline \multicolumn{1}{|c|}{ Combined Uncertainty } & $u_{t}$ & $1.00 \mathrm{E}-08(\mathrm{~s})$ \\
\hline
\end{tabular}




\section{CHAPTER 3: COMPUTATIONAL FLUID DYNAMICS}

\subsection{Computational Mesh and Solver Considerations}

Both two-dimensional and three-dimensional simulations were performed to study the effects of dynamic roughness on the development of the LEV. The NACA 0012 airfoil was again chosen to study based on availability of data and the fact that it was used in previous research associated with the current study. Also, there was a desire to compare the computational simulations with the experimental PIV data. The first consideration when developing the computational mesh besides grid density and structure was to understand the manner in which the pitching should be modeled. The commercial software Fluent was used for all computational studies, which presented three different ways to model a pitching airfoil: 1) Rotate the airfoil within a stationary fluid domain, 2) rotate the fluid domain while keeping the airfoil fixed, and 3) apply a varying velocity vector on the inlet boundary. Mesh motion and varying the velocity vector is accomplished by applying a Fluent User Defined Function (UDF). A UDF is a function written using $C$ architecture and built-in macros provided by Fluent. The UDF used to rotate a domain or airfoil is documented in APPENDIX A, and the UDF used to apply a variable velocity vector over the inlet boundary is documented in APPENDIX B. All three processes were evaluated to find a suitable method for this particular application.

Fluent offers two different methods for mesh motion in the application of a pitching airfoil; "sliding mesh" and "dynamic mesh". In the sliding mesh technique two cell zones are created in such a way to be bounded by at least one "interface zone" where it meets the opposing cell zone. The interface zones of the two adjacent cell zones are associated with one another to form a "mesh interface". The two cell zones will move relative to each other along the mesh interface. As the cell zones slide along the mesh interface, node alignment is not required. This node misalignment is highlighted in Figure 3.1. The dynamic mesh technique recalculates the position of nodes within a defined zone based on the motion of the zone and the parameters entered in the solver. The update of the volume mesh is handled automatically by FLUENT at each time step based on the new position of the zone boundaries [51]. To pursue both methods a twodimensional mesh was developed that could use either method of mesh motion. 


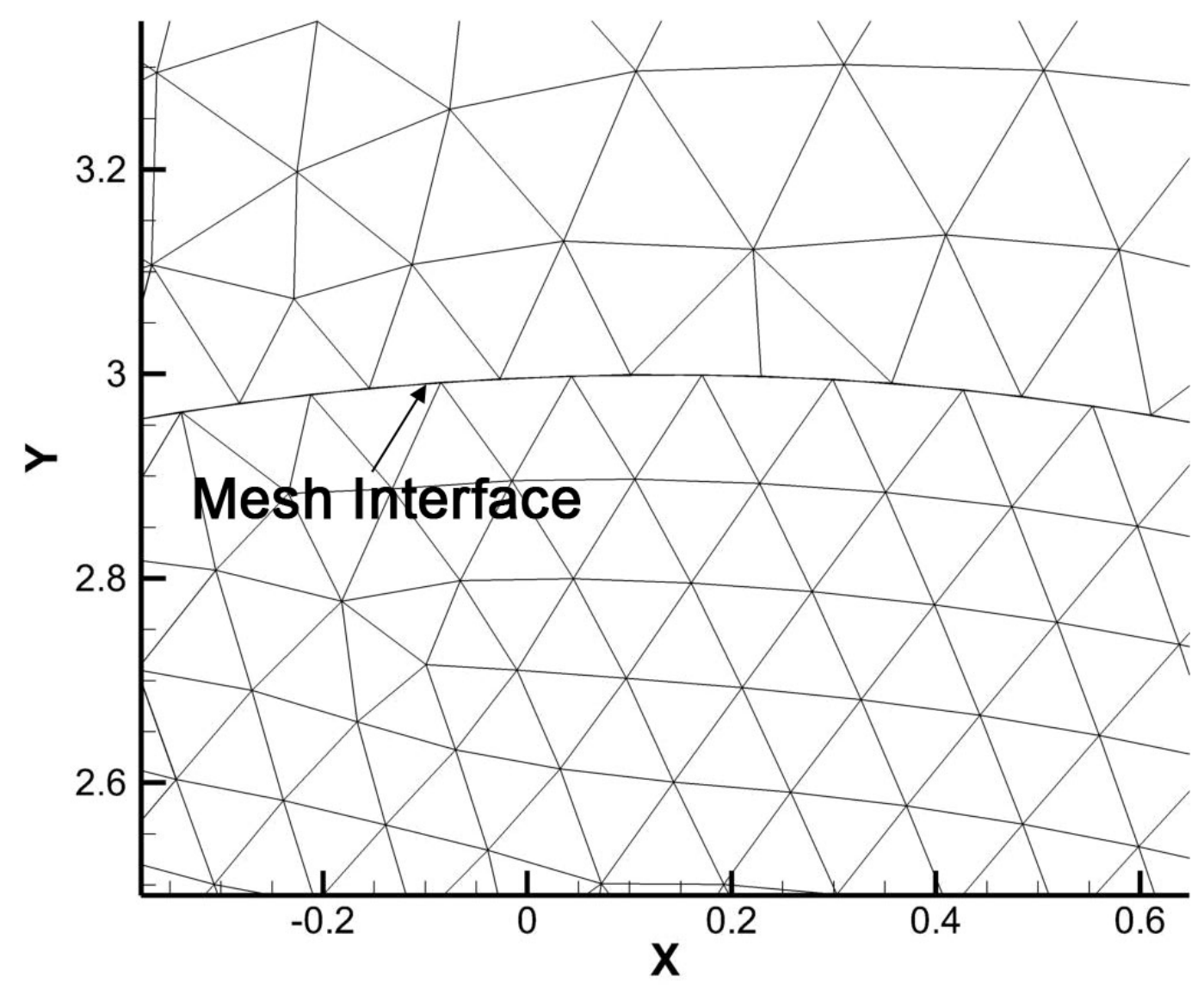

Figure $3.1 \quad$ Nodes do not have to be aligned along a mesh interface using the sliding mesh technique.

Figure 3.2 and Figure 3.3 show the preliminary mesh used for the purpose of mesh motion evaluation, which has a basic structure of a structured C-grid wrapped in an unstructured O-grid. The inlet boundary is placed 12 chord lengths ahead of the airfoil and the outlet is 14 chord lengths aft of the chord. The top and bottom boundaries are both 10 chord lengths from the airfoil's mean chord line. This domain size was chosen to limit the domain size for computational efficiency while accurately representing the flow near the airfoil during the pitching maneuver. The mesh has a total of 146,748 nodes (149,635 cells) and comprises a structured zone within an unstructured zone. The structured zone allows for accurate and efficient calculations as well as excellent mesh smoothing needed while activating the dynamic roughness elements on the surface of the airfoil. The Dynamic Roughness (DR) Zone needs to be identified separately within Fluent as a deforming zone to allow mesh smoothing during DR actuation. The Re-meshing Zone serves two purposes: if using the sliding mesh method to pitch 
the airfoil, this identifies the mesh interface on which to slide; if using the dynamic mesh method this identifies the zone which Fluent will apply re-meshing and smoothing as either the outer or inner zone rotates. Each roughness element is comprised of 12 nodes, with a uniform spacing of 0.000272c. Aft of the roughness elements are 200 more nodes to form the rest of the airfoil, the spacing ranges from $0.000272 \mathrm{c}$ near the roughness elements to $0.0005 \mathrm{c}$ at the trailing edge. The distribution becomes coarse at the midpoint of this edge then finer as it nears the trailing edge. The spacing normal to the airfoil surface is $0.00005 \mathrm{c}$ and grows beyond the boundary layer. This spacing normal to the airfoil surface provides about 50 nodes within the boundary layer at the midchord location with an angle of attack of 0 degrees. The top and bottom surfaces of the airfoil are symmetric. The structured zone extends to $0.5 \mathrm{c}$ in front of the airfoil and $1 \mathrm{c}$ aft of the airfoil. Beyond this an unstructured zone gradually coarsens as it moves towards the domain boundary. This reduces the number of nodes required in the mesh. A laminar solver is used for all computational studies done with regards to this research.

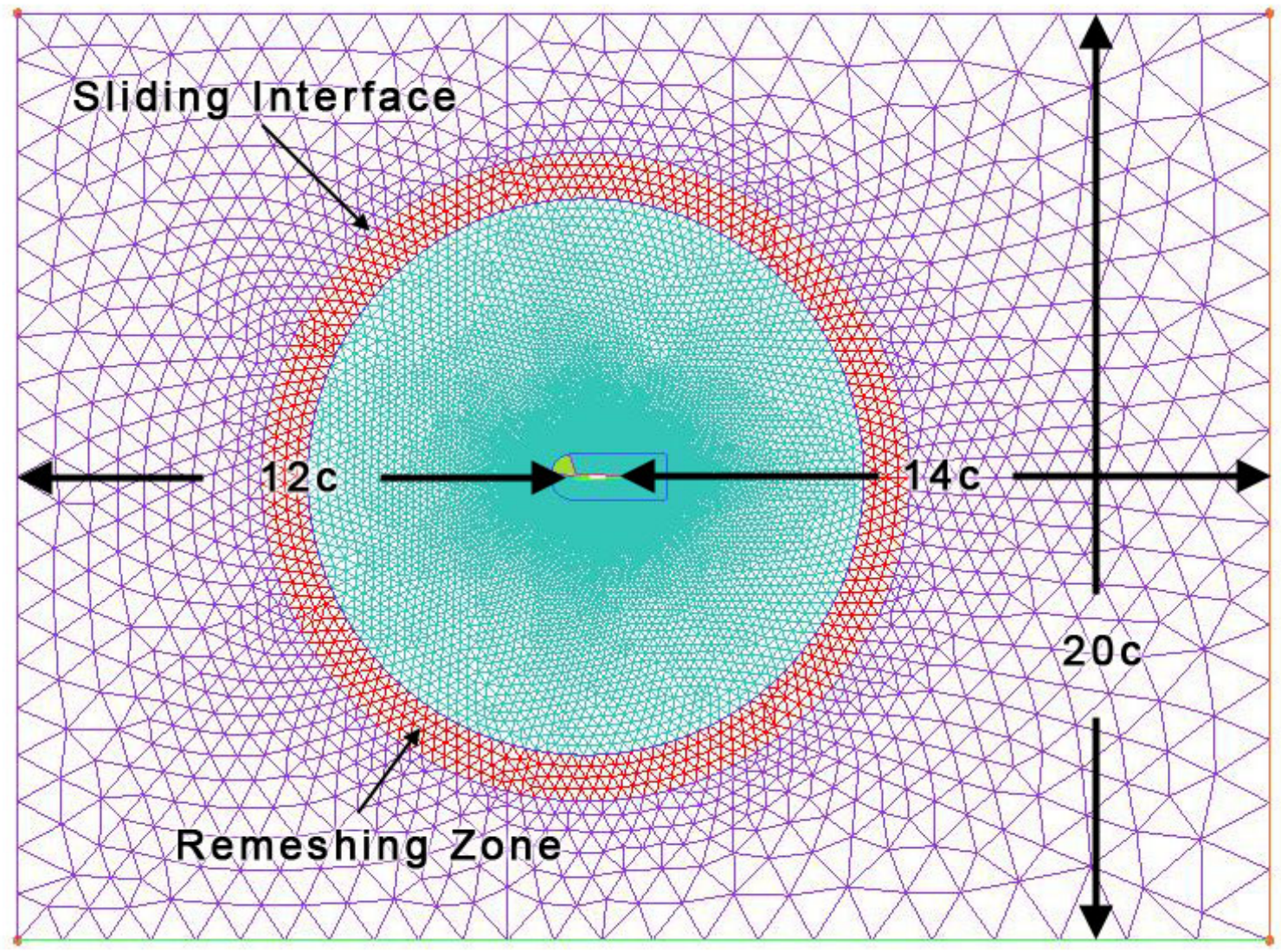

Figure 3.2 Mesh used for dynamic stall simulation. Allows for use of three different methods simulating a pitching airfoil. 


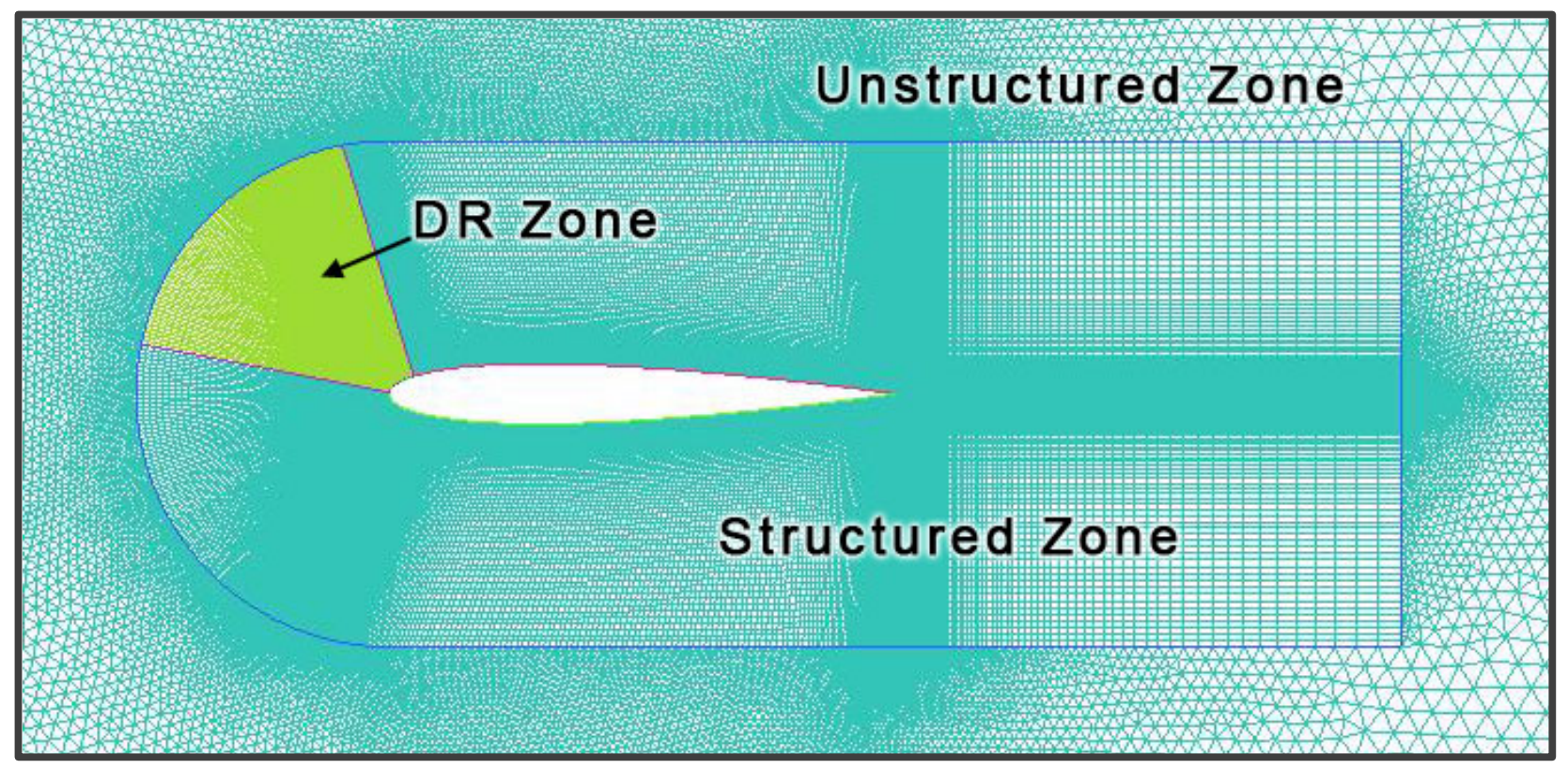

Figure 3.3 View of mesh highlighting the structured zone within unstructured zone as well as the Dynamic Roughness Zone above the roughness elements on the airfoil surface.

The first evaluation was to decide which method of pitching modeling should be used, either the sliding mesh approach or the dynamic mesh technique. To accomplish this the time to complete a clean pitching simulation was observed for each approach. It was found that the sliding mesh technique took almost twice the amount of time that the dynamic mesh approach needed to complete the simulation. This observation agrees with what is stated in the FLUENT Theory Guide which states, "the sliding mesh model is the most accurate method for simulating flows in multiple moving reference frames, but also the most computationally demanding” [51]. It is important to note that no significant difference in flow solution was observed between the two methods. Comparison of streamline plots and surface pressure distributions were performed to check correlations between the two mesh techniques, as shown in Figure 3.4 and Figure 3.5. The flow and pitching parameters used for this comparison were those used by [17], which are displayed in Table 3.1. The conditions used in Choudhuri, et al.'s analysis are quite appropriate for comparison due to the fact that it was a low Reynolds number flow ( $\operatorname{Re}=10,000)$ and twodimensional, which is the regime of focus for this research. A variety of variables are introduced and defined to characterize the pitching motion. These variables are used throughout the published research highlighted previously. A non-dimensional pitch rate is introduced, which corresponds to a functional form of pitch rate that varies with time to produce a smooth 
acceleration of the airfoil to its asymptotic pitching rate, $\Omega_{0}$. This non-dimensional pitch rate is calculated using (4).

$$
\Omega_{0}^{+}=\frac{\Omega_{0} c}{U_{\infty}}
$$

where $c$ is the airfoil chord length and $U_{\infty}$ is the freestream velocity. The pitching motion is defined by

$$
\Omega(t)=\Omega_{0}\left[1-e^{-4.6 t / t_{0}}\right]
$$

where $\Omega$ is the pitch rate in $\mathrm{rad} / \mathrm{s}, t_{0}$ is the time at which $\Omega$ has reached $99 \%$ of the asymptotic value, $\Omega_{0}$. The time to reach $\Omega_{0}$ can be calculated using (6).

$$
t_{0}=\frac{0.5 c}{U_{\infty}}
$$

Very little difference is observed between the two methods. The most notable difference is the surface pressure distribution near the trailing edge shown in Figure 3.5. Since the area of interest is the near the leading edge, this discrepancy in surface pressure near the trailing edge is negligible. With this knowledge the dynamic mesh approach was used to model both the airfoil rotating in a fixed domain as well as the domain rotating around a fixed airfoil.

Table 3.1 Pitching parameters used for mesh comparison, pitching method comparison, and validation with established research.

\begin{tabular}{|l|c|}
\hline \multicolumn{1}{|c|}{ Parameter } & Value \\
\hline Chord Reynolds number, $R e_{c}$ & 10,000 \\
\hline Chord length, $c$ & $0.5 \mathrm{~m}$ \\
\hline Non-dimensional pitch rate, $\Omega_{0}^{+}$ & 0.2 \\
\hline
\end{tabular}




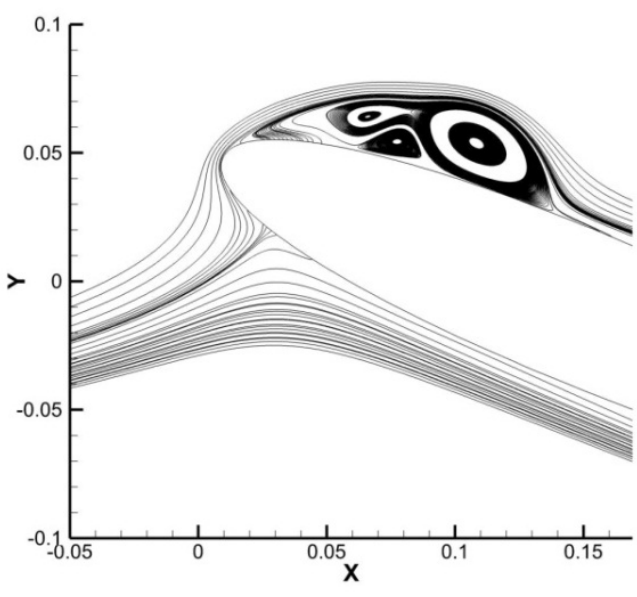

(a)

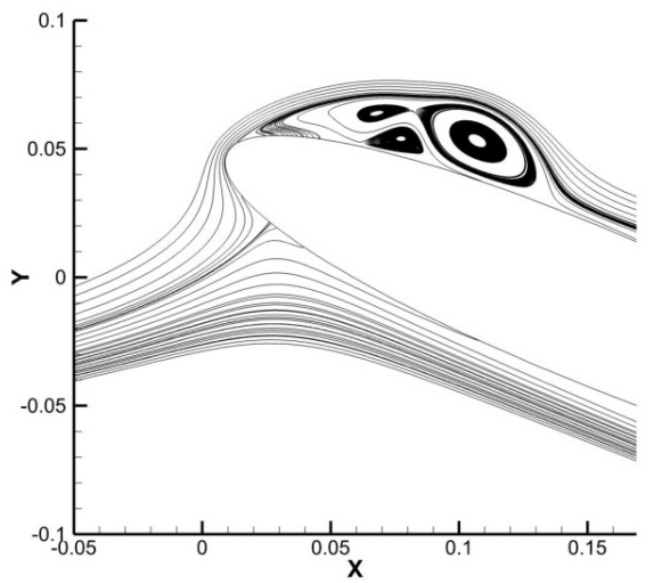

(b)

Figure 3.4 Comparison between streamline results provided by the (a) dynamic mesh method and the (b) sliding mesh method.

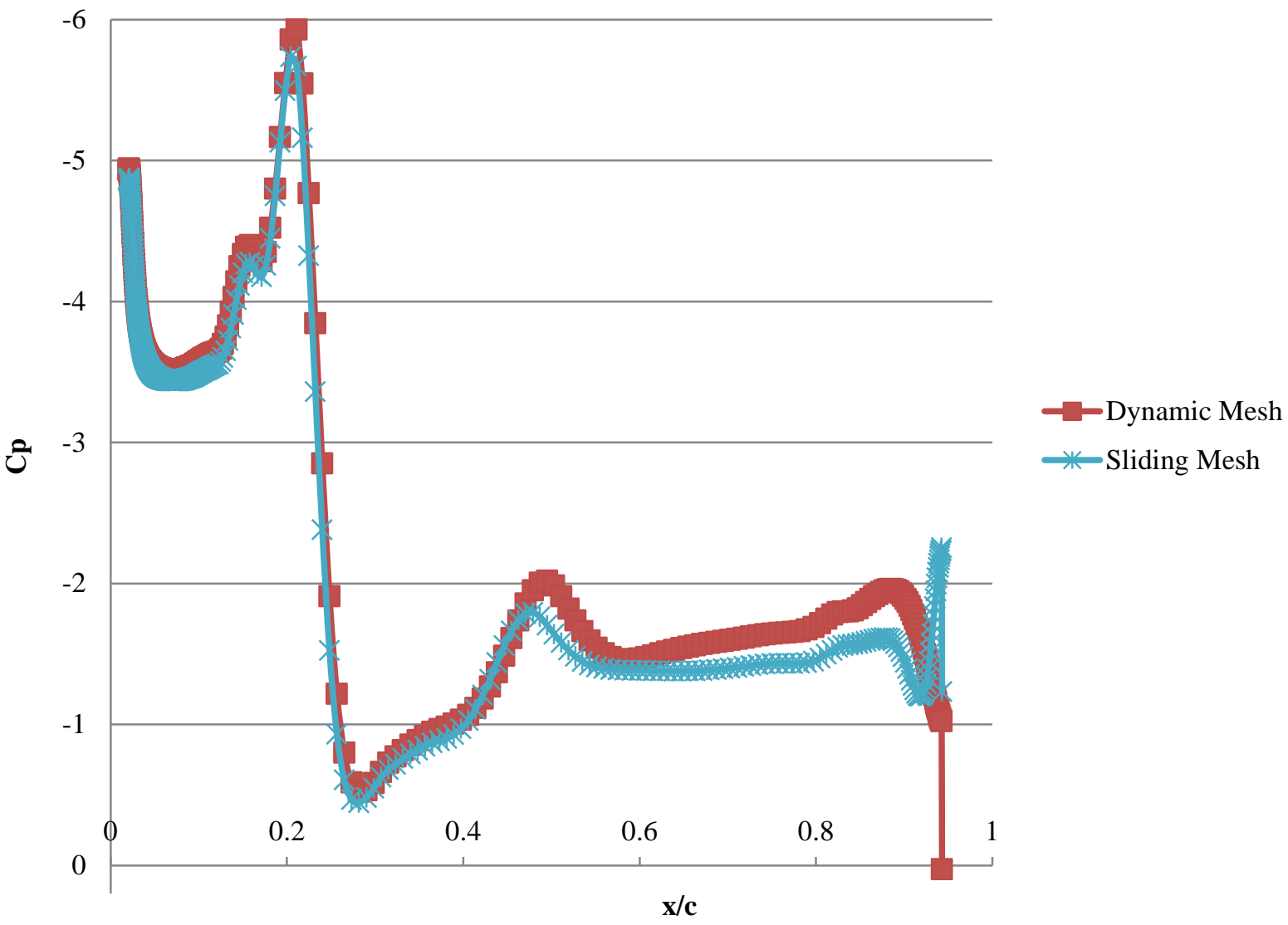

Figure 3.5 Computed airfoil surface pressure distribution for the dynamic mesh method and sliding mesh method when airfoil is at $22.5^{\circ}$ angle of attack. 
The next consideration is which technique should be used to model the pitching motion; (1) rotating airfoil in a stationary domain, (2) fixed airfoil with a rotating domain, or (3) fixed airfoil and fixed domain with a varying velocity vector at the inlet. The same mesh and flow characteristics were used for this study as well. The main comparison tool is to match streamline plots with the work done by [17]. The main point of comparison is the emergence of three recirculating regions along the upper surface of the airfoil at an angle of attack of $22.5^{\circ}$. Figure 3.7 shows streamlines of the flow around a rapidly pitching airfoil at a time corresponding to $\alpha=$ $22.5^{\circ}$ as measured by the angle created by the intersection between the mean chord line and the normal vector to the inlet boundary. This method of measurement was chosen for consistency between methods. The angle of attack for the velocity vector method was prescribed at the inlet boundary.

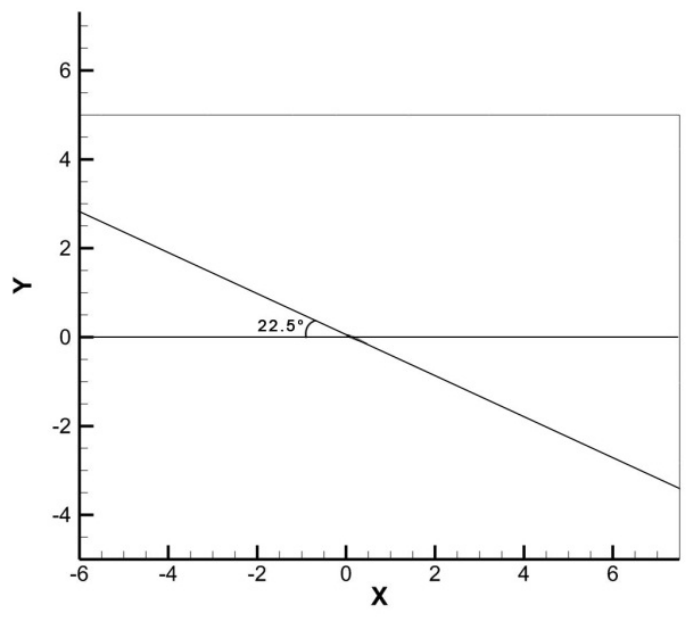

(a)

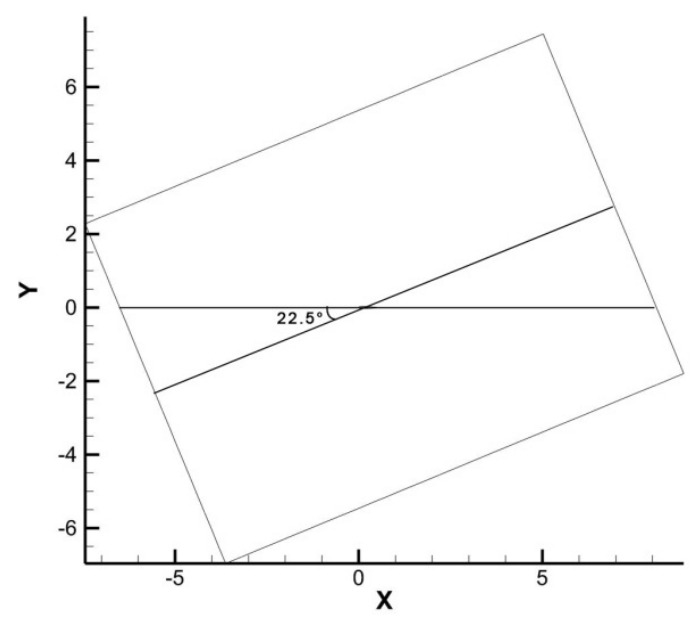

(b)

Figure 3.6 Representation of how angle of attack was calculated for (a) rotating airfoil and (b) rotating boundary with stationary airfoil. 


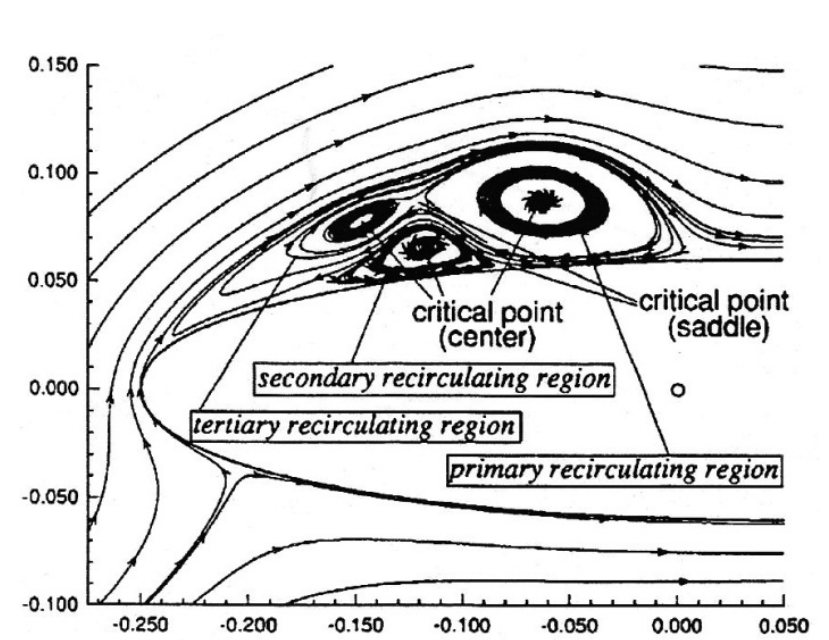

(a)

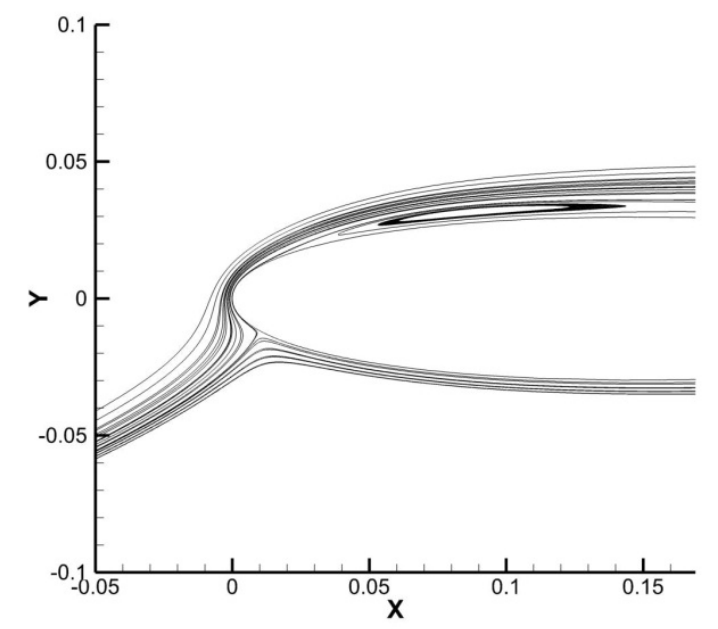

(c)

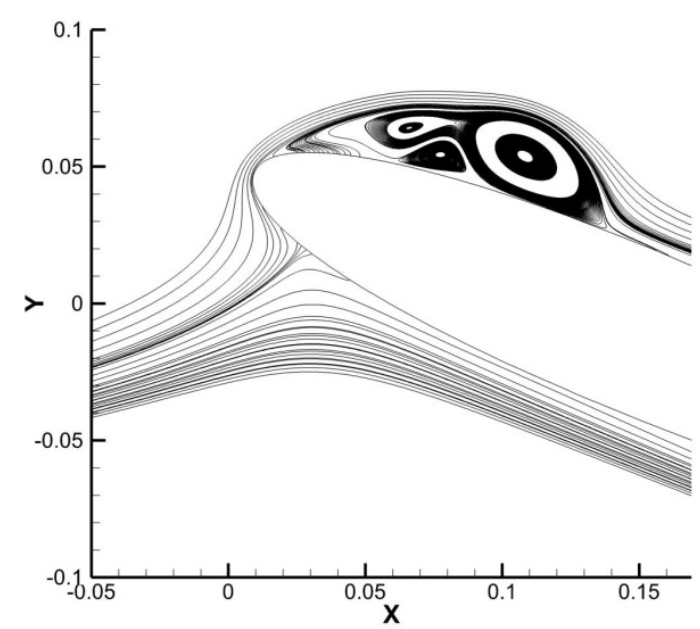

(b)

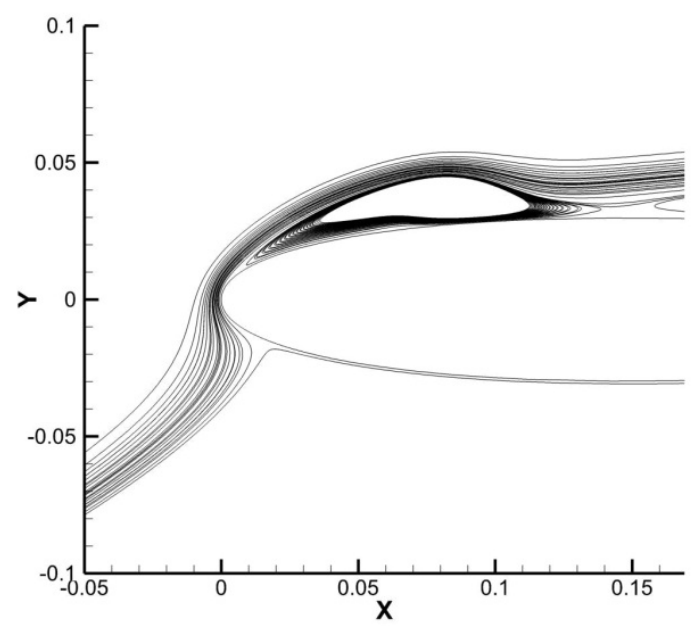

(d)

Figure 3.7 Two-dimensional streamlines of a rapidly pitching airfoil at $22.5^{\circ}$ angle of attack for (a) Choudhuri, et al. results; (b) airfoil pitching about $25 \%$ chord; (c) airfoil fixed with domain rotating about $25 \%$ chord; and (d) variable velocity vector prescribed over velocity inlet.

As shown in Figure 3.7, there are significant differences between pitching methods. In the simulation where the domain is kept stationary as the airfoil is rotated the results closely match those of [17]. Although the other two methods show different results, they both eventually develop the LEV, albeit at higher "measured” angles of attack.

It is found that the formation of the LEV occurs at $28.79^{\circ}$ for the boundary rotation method and at $24.77^{\circ}$ for the variable velocity vector method. It is hypothesized in the boundary rotation method there is a lag time associated with the time needed for the rotation of the velocity inlet to 
propagate throughout the domain. As can be seen in Figure 3.8 the streamlines do not stay perpendicular to the inlet throughout the domain for the rotating boundary method. This is thought to reduce the "effective" angle of attack sensed by the airfoil. The source of variation for the velocity vector method is thought to be due to the fact that there is no way to identify a pitching axis when the velocity is prescribed at the inlet, as well as the time of propagation required for fluid at the inlet to reach the airfoil. As stated previously by [14], pitching axis has significant effects on LEV formation. In addition, this method also neglects the inertial term of the governing equations. Although neglecting this term will alter the "absolute" analysis, it will still qualitatively show the trend of separation, reattachment, and recirculation which is sufficient for comparison for clean and dynamic roughness cases.

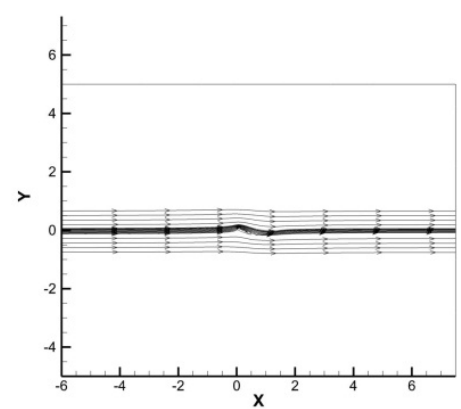

(a)

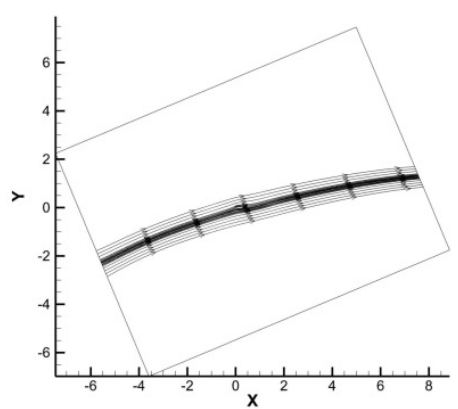

(b)

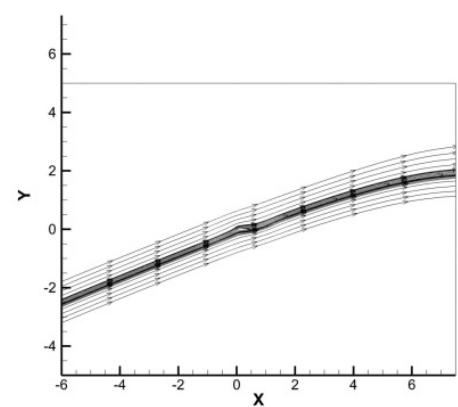

(c)

Figure 3.8 Streamlines at $22.5^{\circ}$ for a (a) pitching airfoil in a stationary domain, (b) rotating domain with a fixed airfoil, and (c) variable velocity vector.

Although it is obvious from the airfoil pitching method study that the rotating airfoil in a stationary domain best matched previous established research, it was not chosen as the method to be used for this research. The reason is due to the manner in which the dynamic roughness elements are modeled in FLUENT using the UDF outlined in APPENDIX C. Defined in the UDF code is the node locations in terms of $\mathrm{x}$ and $\mathrm{y}$ coordinates of the domain that prescribes the start and end of each roughness element. The UDF then loops through each node between these points and prescribes a new position, again in terms of $\mathrm{x}$ and $\mathrm{y}$ coordinates of the entire domain, based on the current time step, inputted actuation frequency, and inputted element amplitude. Therefore, to model the dynamic roughness elements on a moving airfoil the motion of the airfoil itself needs to be accounted for in the node placement given by the UDF. All attempts to accomplish this were unsuccessful. Even though the double precision solver was used, it still 
appeared to give enough truncation error to misalign the dynamic roughness element nodes. For this reason it was decided to use the variable velocity vector method model the pitching motion of the airfoil. This not only solved the truncation error problem just described, since the airfoil is not moving, but it also allows for a fully structured mesh to be utilized with no need for a Remeshing Zone, as is needed in the pitching method in which the domain is rotated about a fixed airfoil. This decreases computational time, especially in three-dimensional simulations.

It was decided to limit CFD simulations to a Reynolds number of 150,000 due to the laminar solver being used for this study. Although there is most certainly transition and turbulence occurring at this flow velocity, in the region of interest near the leading edge there is laminar separation, which is best modeled by the laminar solver. In the research performed by Gall et al., the laminar solver was also used [6]. The numerical studies using the laminar solver compared well with the experimental work, as is the case in the current study. Although the work described here has a much more unsteady character, it is still hypothesized that the laminar solver is suitable to evaluate the separation and reattachment near the leading edge. With this approach the computational results further downstream on the airfoil should be viewed with skepticism.

A mesh density and temporal grid independence study was conducted at this Reynolds number. An initial mesh consisting of 103 nodes along the airfoil and 120 nodes normal to the airfoil was developed. The mesh was then refined in 50\% increments in both directions until no noticeable changes in solution were observed. Since this is an unsteady flow, the characteristics used for solution independence is the initiation of recirculation and the structure of the LEV at a higher angle of attack. Figure 3.9 illustrates these observations and no change is observed from Mesh 3 to Mesh 4. Negligible differences are again observed between Mesh 3 and Mesh 4 when comparing the LEV structure at higher angles of attack, as shown in Figure 3.10. 
Table 3.2

Properties of different grids used for refinement study.

\begin{tabular}{l|ccccc|} 
Mesh & $N_{S}$ & $N_{N}$ & $\Delta x / c$ & $\Delta y / c$ & $N_{B L}$ \\
\hline 1 & 103 & 120 & 0.0022 & $4 \mathrm{e}-5$ & 39 \\
2 & 153 & 180 & 0.0014 & $4 \mathrm{e}-5$ & 53 \\
3 & 180 & 206 & 0.0010 & $4 \mathrm{e}-5$ & 66 \\
4 & 257 & 240 & 0.0008 & $7 \mathrm{e}-7$ & 118 \\
\hline
\end{tabular}

$N_{S} \equiv$ Number of nodes along airfoil's surface.

$N_{N} \equiv$ Number of nodes normal to airfoil's surface.

$\Delta x / c \equiv$ Minimum node spacing along airfoil's surface, non-dimensionalized by chord length.

$\Delta y / c \equiv$ Minimum node spacing normal to airfoil's surface, non-dimensionalized by chord length.

$N_{B L} \equiv$ Number of nodes in boundary layer measured normal to airfoil surface at midchord at $0^{\circ}$ angle of attack.

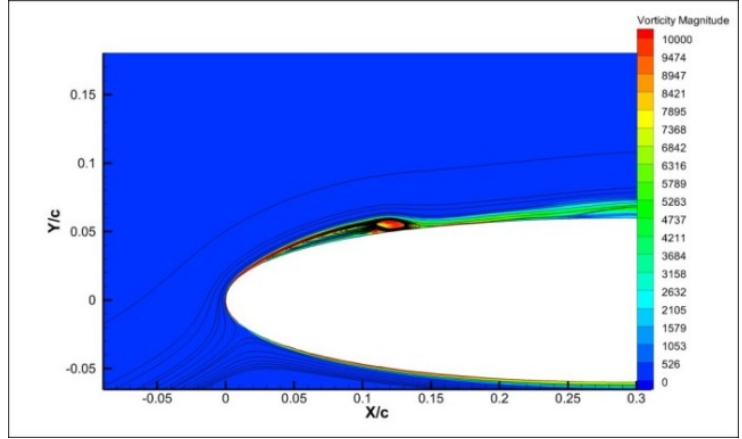

(a)

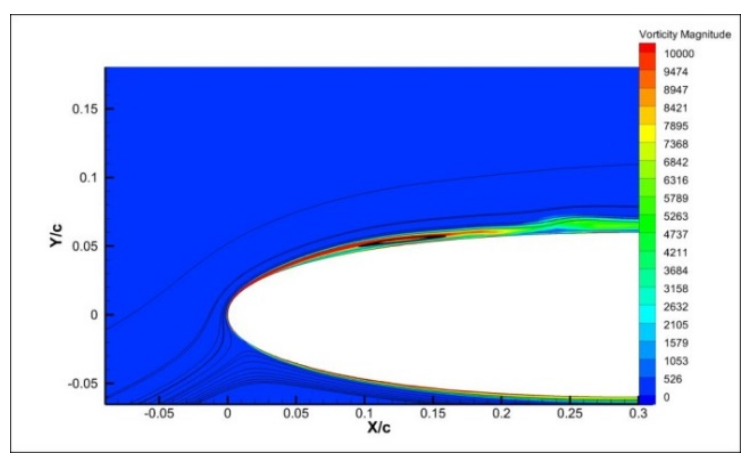

(c)

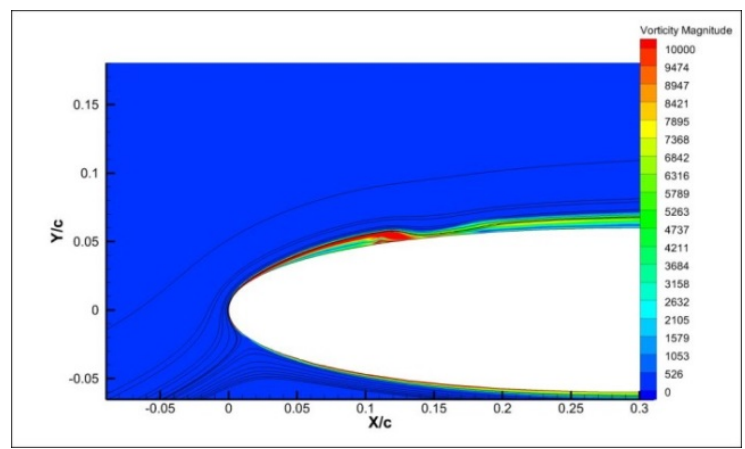

(b)

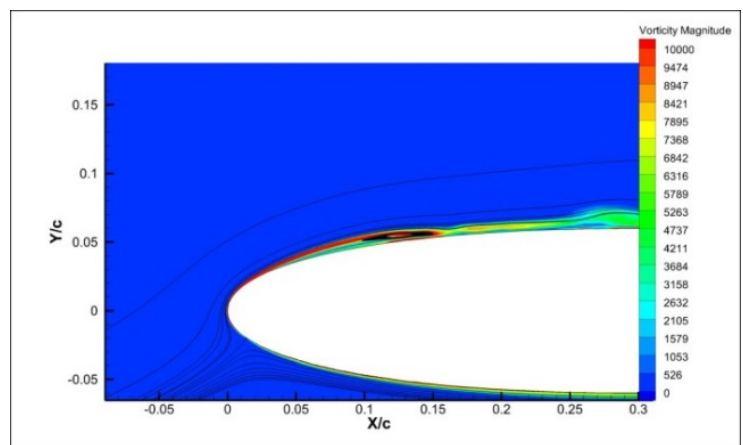

(d)

Figure 3.9 Grid refinement study observing initiation of LEV development for (a) Mesh 1, (b) Mesh 2, (c) Mesh 3, and (d) Mesh 4. 


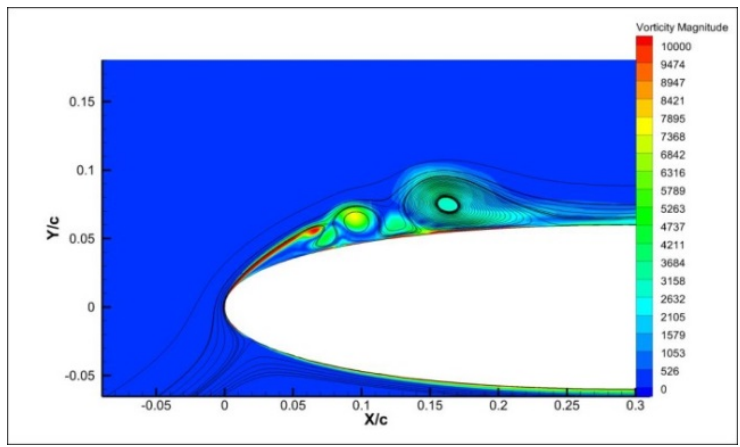

(a)

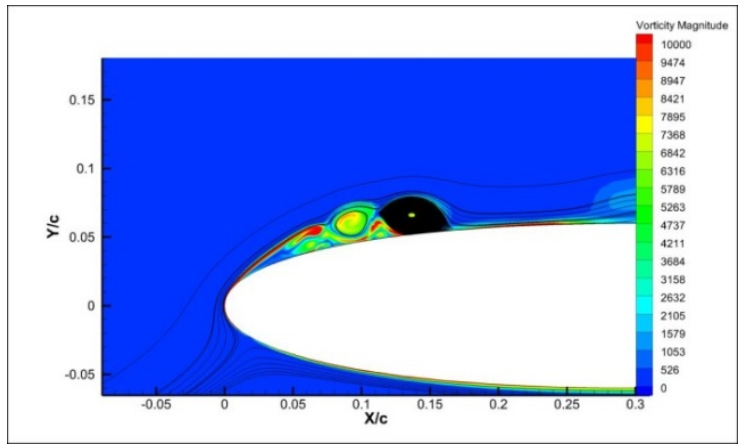

(c)

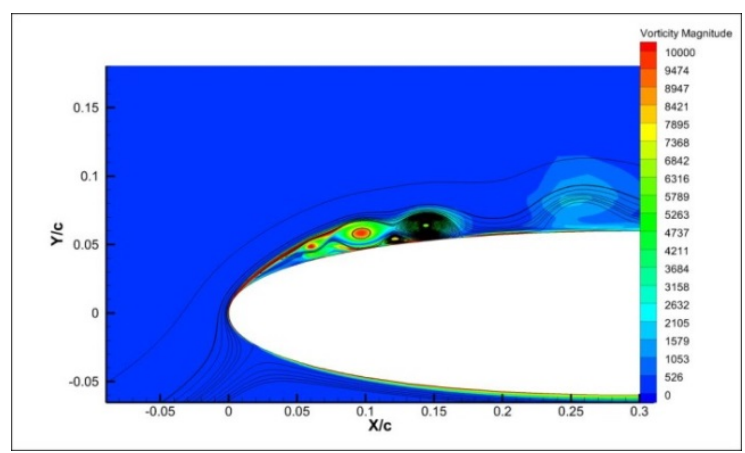

(b)

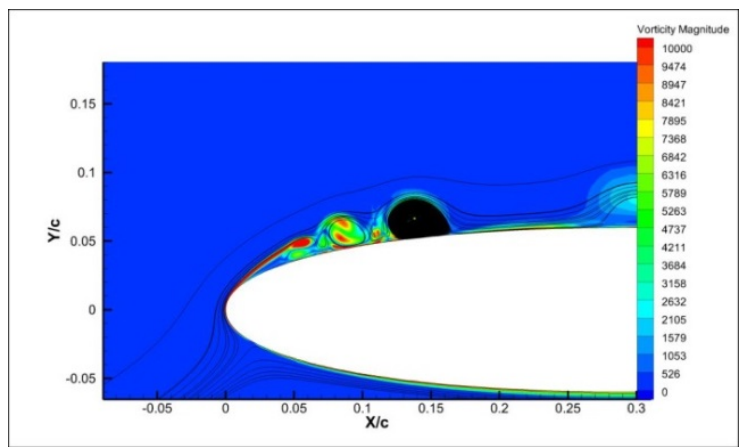

(d)

Figure 3.10 Grid refinement study observing LEV structure for (a) Mesh 1, (b) Mesh 2, (c) Mesh 3, and (d) Mesh 4.

To study temporal solution independence a non-dimensional time step was utilized. The form is given in (7).

$$
\Delta t^{*}=\frac{\Delta t U_{\infty}}{c}
$$

The same approach used to investigate mesh density independence is used to study temporal independence. Figure 3.11 displays the onset on recirculation for four different time steps. There is almost no difference between solutions. More significant differences can be seen in Figure 3.12 which characterizes the structure of the LEV at a higher angle of attack. An interesting observation made during the temporal study is that it is not simply the structure of the LEV that is different at different time step sizes, but vortex shedding does not become apparent until a non-dimensional time step of 0.001 is used. 


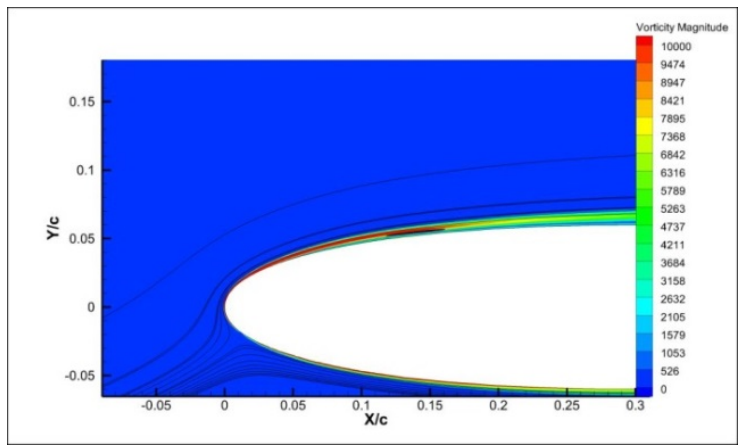

(a)

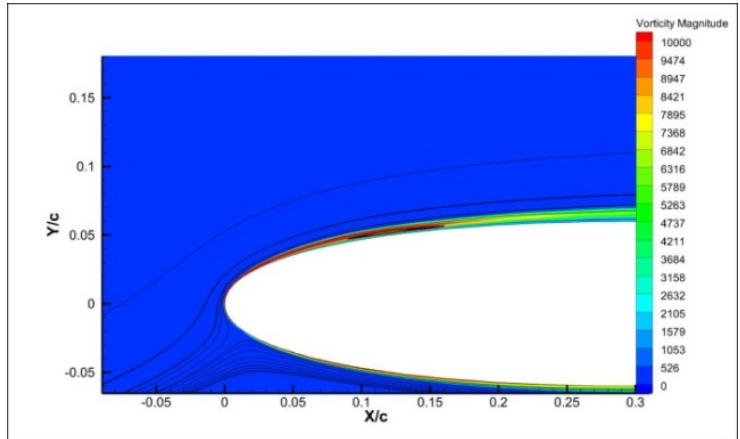

(c)

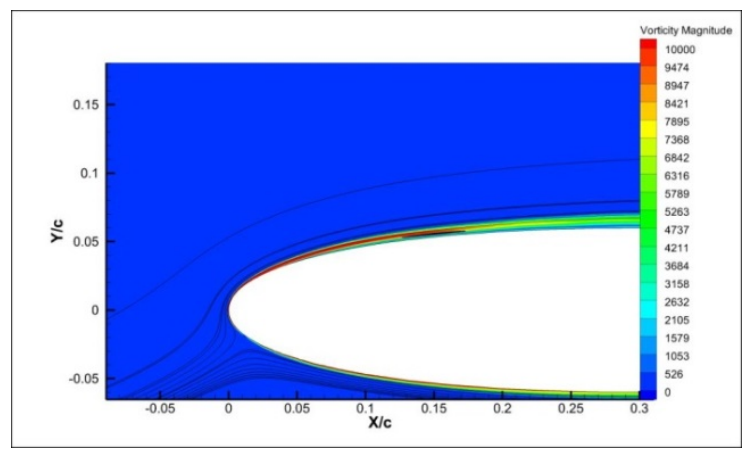

(b)

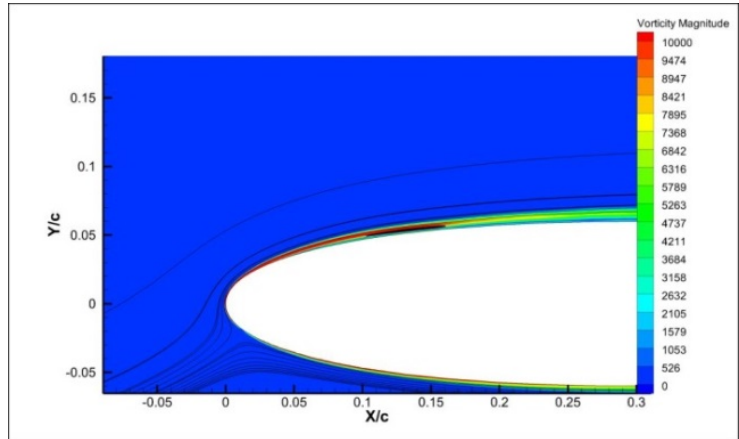

(d)

Figure 3.11 Temporal refinement study observing initiation of LEV development for a $\Delta t^{*}=$ (a) 0.01, (b) 0.005 , (c) 0.001, and (d) 0.0005 .

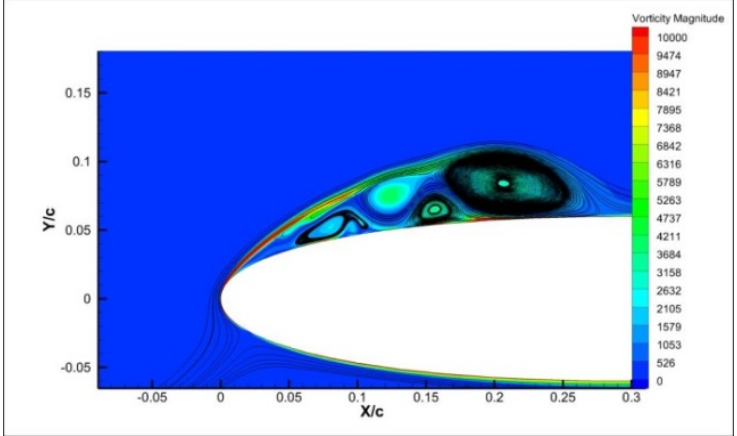

(a)

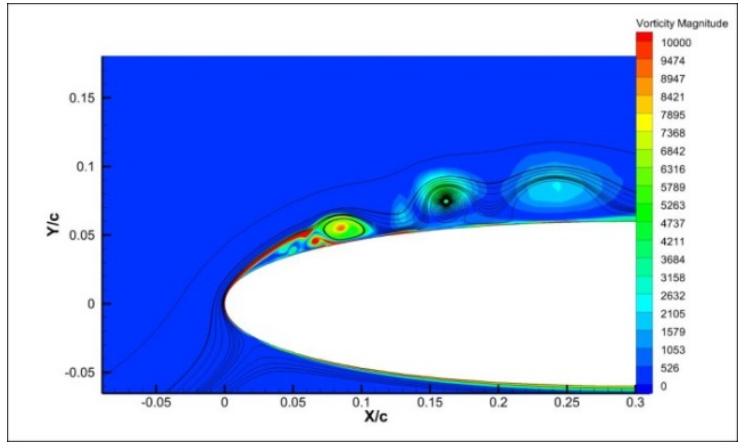

(c)

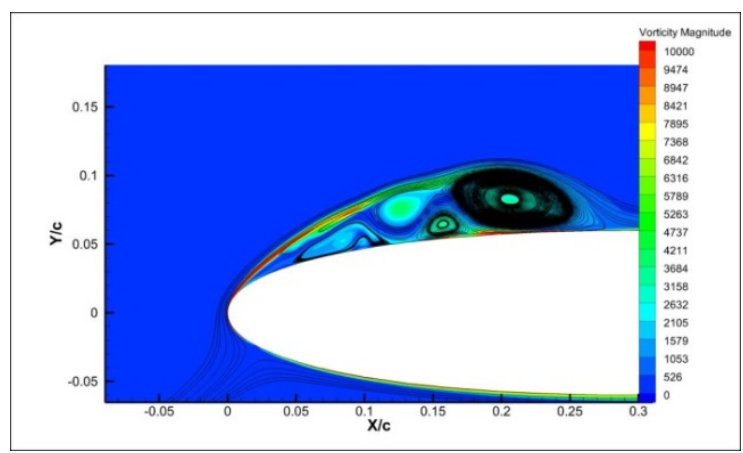

(b)

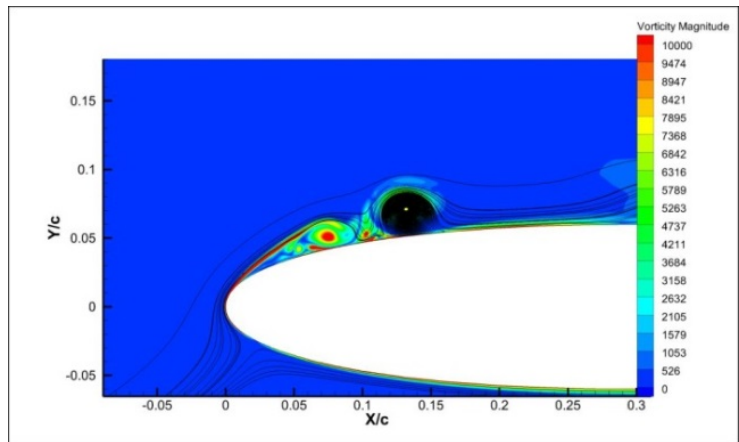

(d)

Figure 3.12 Temporal refinement study observing vortex shedding for a $\Delta t^{*}=$ (a) 0.01 , (b) 0.005 , (c) 0.001 , and (d) 0.0005 . 
Based on the grid and temporal independence study Mesh 3 was used with $\Delta t^{*}=0.001$ for all two-dimensional cases. The revised structured C-grid type mesh is shown in Figure 3.13. The mesh spacing over each dynamic roughness element and the leading edge is 0.001c. The mesh spacing over the remaining surface of the airfoil to the trailing edge has a hyperbolic stretching profile that matches the dynamic roughness element spacing, then stretches to a maximum spacing of $0.027 \mathrm{c}$, then shrinks back to $0.001 \mathrm{c}$ at the trailing edge. The normal spacing linearly stretches from 0.00004c at the airfoil surface to 0.02c at the edge of the DR Zone (which is located at a distance $0.65 \mathrm{c}$ normal to the airfoil surface). These specifications provide a mesh with a total of 142,822 nodes, as seen in Figure 3.13 and Figure 3.14.

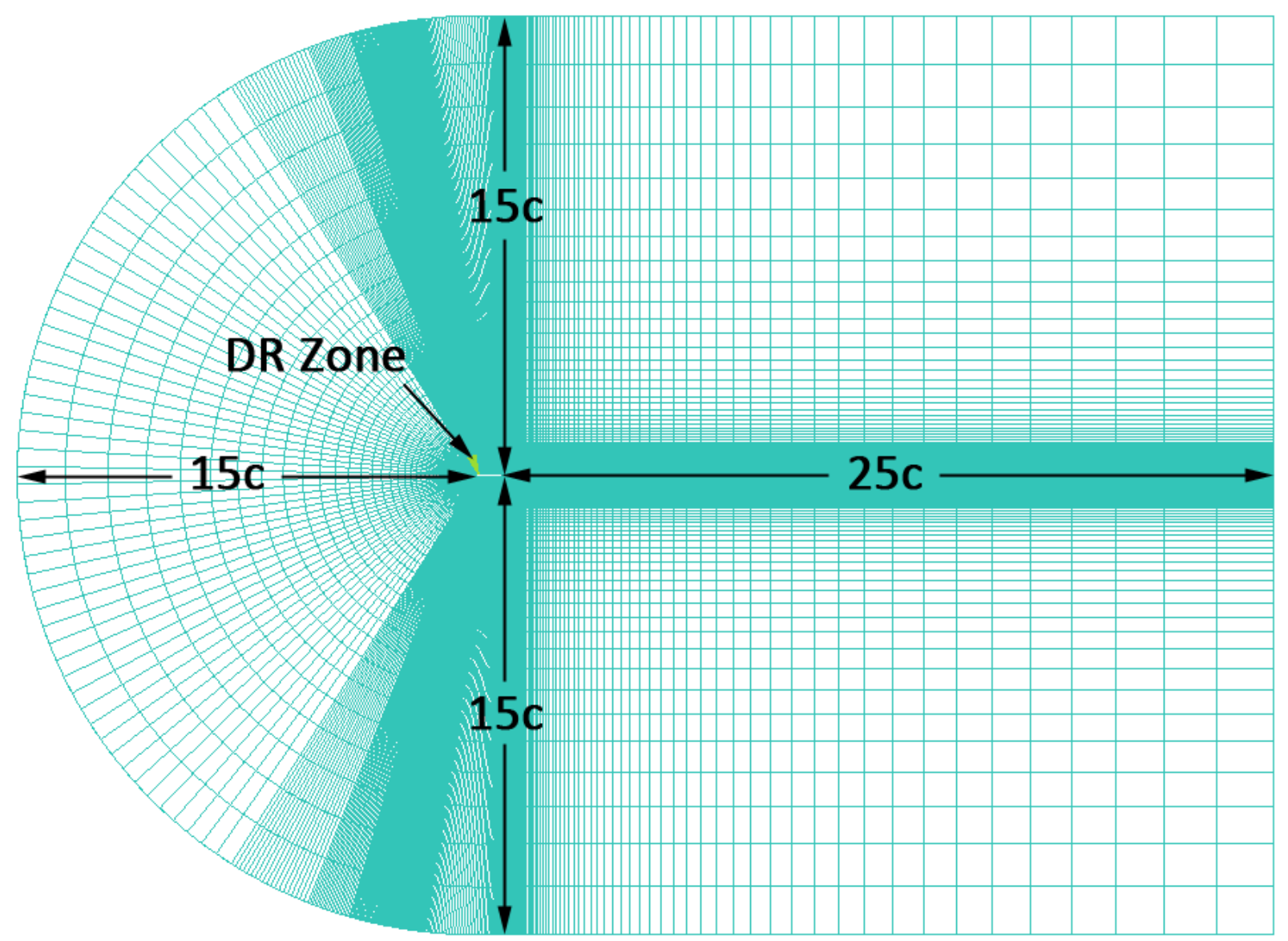

Figure 3.13 Revised two-dimensional grid use for CFD simulations. 


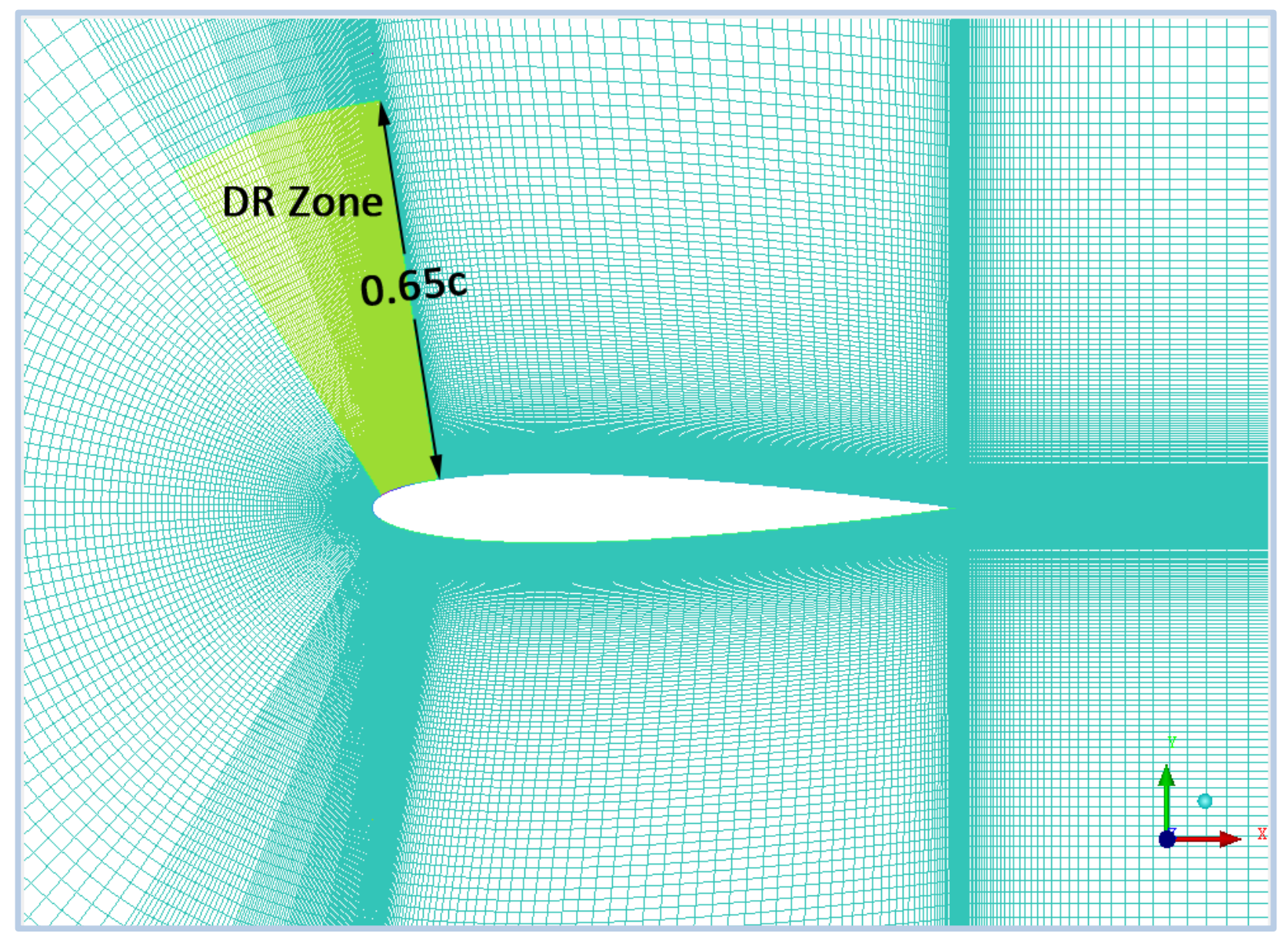

Figure 3.14 Close-up view of the near airfoil region highlighting the extents of the DR Zone.

As described above, to model the dynamic roughness the UDF calculates a new node position based on time, required actuation frequency, and actuation amplitude. In both the threedimensional and two-dimensional simulations the chord-wise length of the dynamic roughness element is dictated by the mesh design. In three-dimensional simulations an additional variable is included in the UDF to dictate the span-wise length of the dynamic roughness element; refer to APPENDIX D. The addition of this variable allows the mesh to be developed without having to define the dynamic roughness element's extent in the span-wise direction. It was previously thought that dynamic re-meshing was necessary to allow for the change in mesh geometry caused by the actuation of a dynamic roughness element [5]. Furthermore, dynamic re-meshing requires tetrahedral cells in the zone identified for re-meshing. This approach tends to result in inefficient unstructured mesh zones within the boundary layer at the location of the dynamic roughness elements. Further investigation of the techniques employed by FLUENT to alter the 
mesh geometry proved this assumption to be false. It was found that if the mesh remains nearly orthogonal to the moving surface then quad shaped cells can be used. Not only this, but the need for dynamic re-meshing is also eliminated and only mesh smoothing is required. This reduces computational time since dynamic re-meshing not only moves nodes to take into account the mesh motion, but also removes or adds nodes as needed to fulfill the mesh density requirements. By removing the necessity for this extra calculation computational time is reduced. Also, the use of structured quad cells within the boundary layer provides efficient meshes for computational studies and provides the capability of large dynamic roughness amplitudes that were not attainable with the unstructured approach. The evolution as well as the structured quad cell structure of both a two-dimensional dynamic roughness element and cluster of three-dimensional dynamic roughness elements can be referred to in Figure 3.15 and Figure 3.16, respectively. 


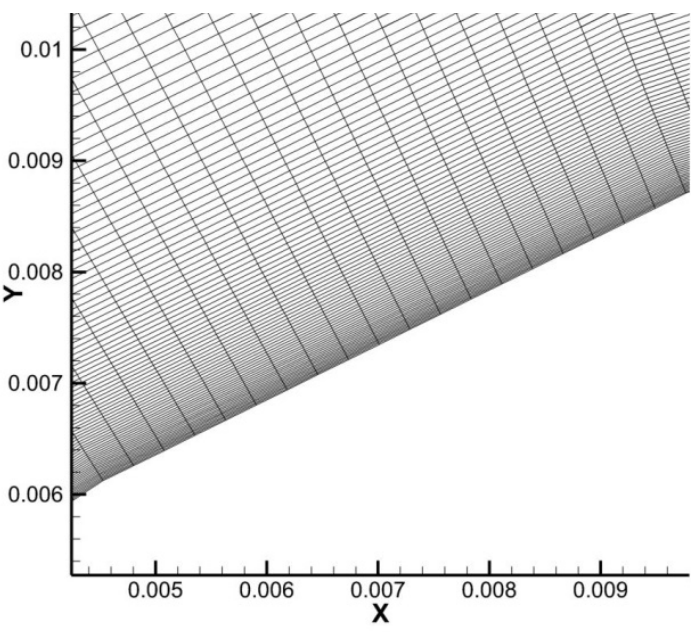

(a)

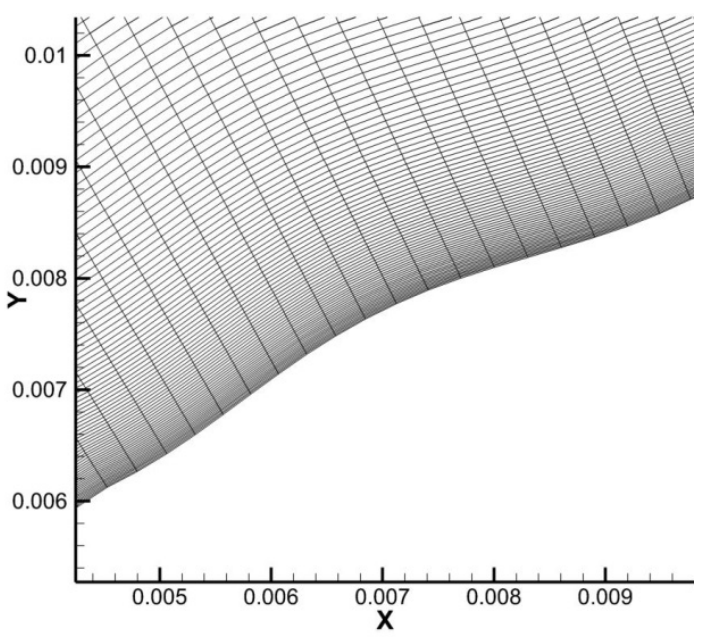

(b)

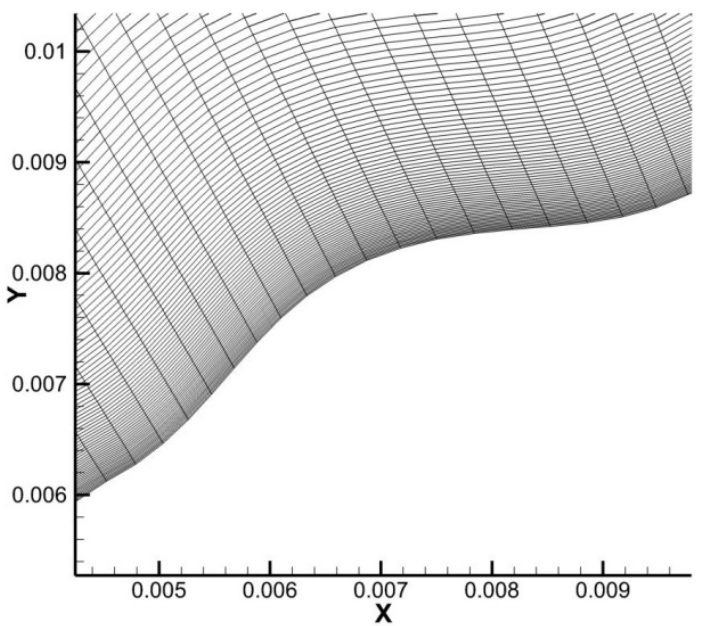

(c)

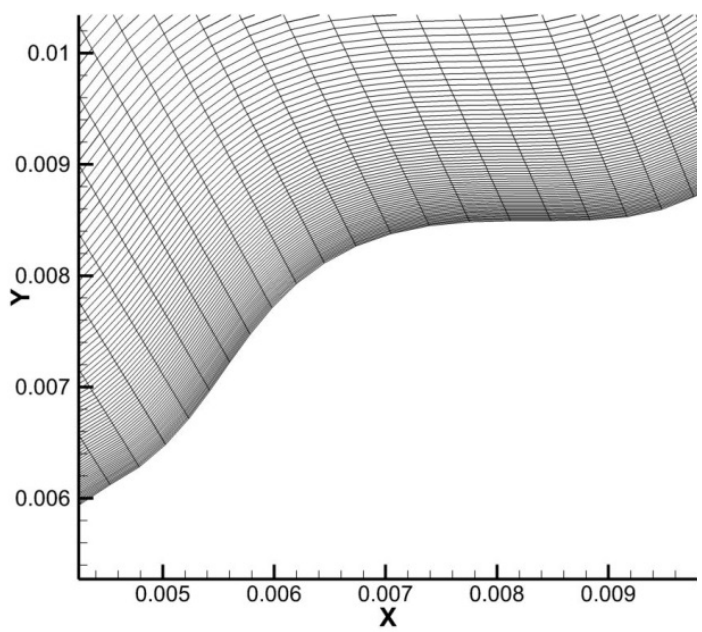

(d)

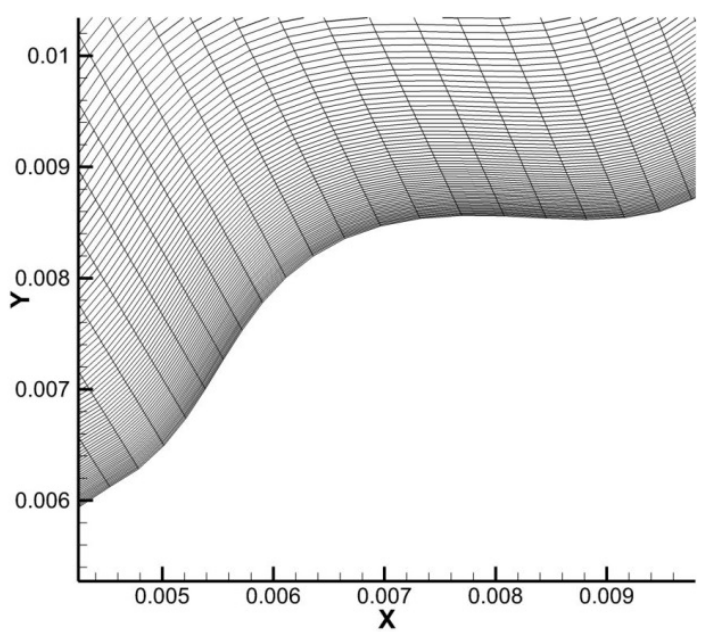

(e)

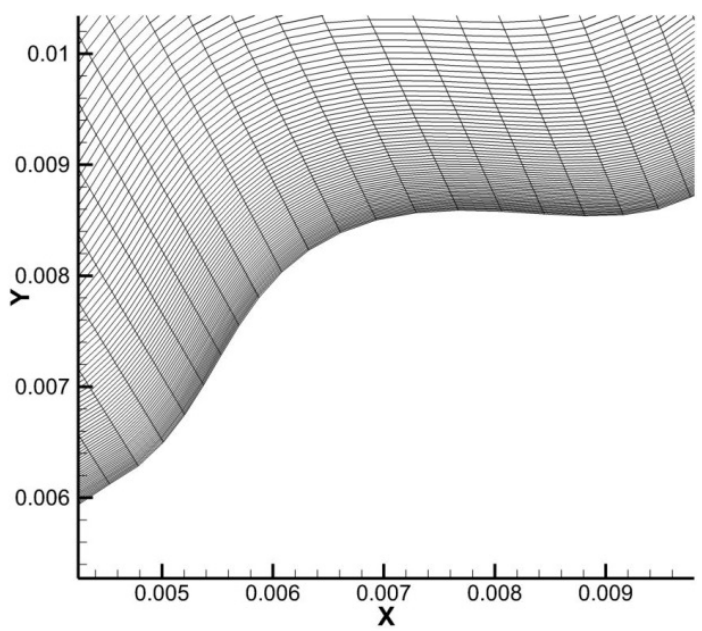

(f)

Figure 3.15 Half a cycle of a two-dimensional dynamic roughness element, starting from a clean surface (a) and reaching its maximum amplitude (f). 


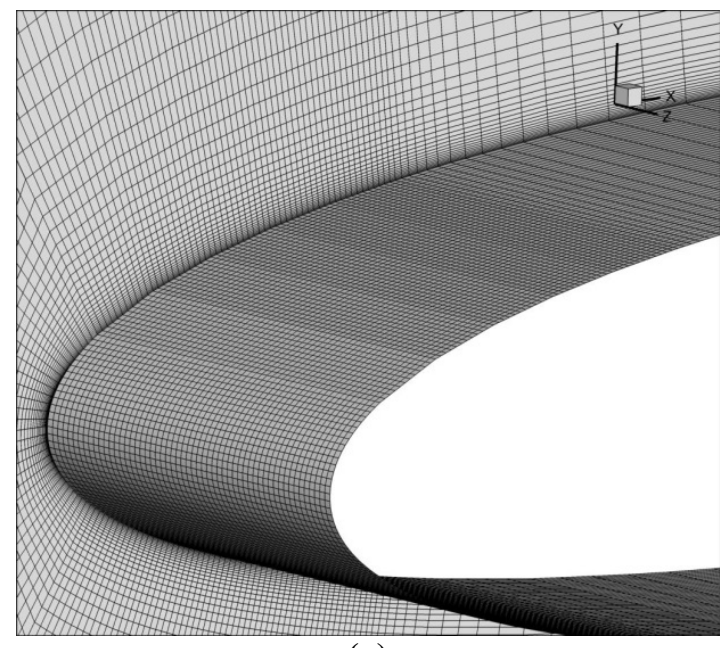

(a)

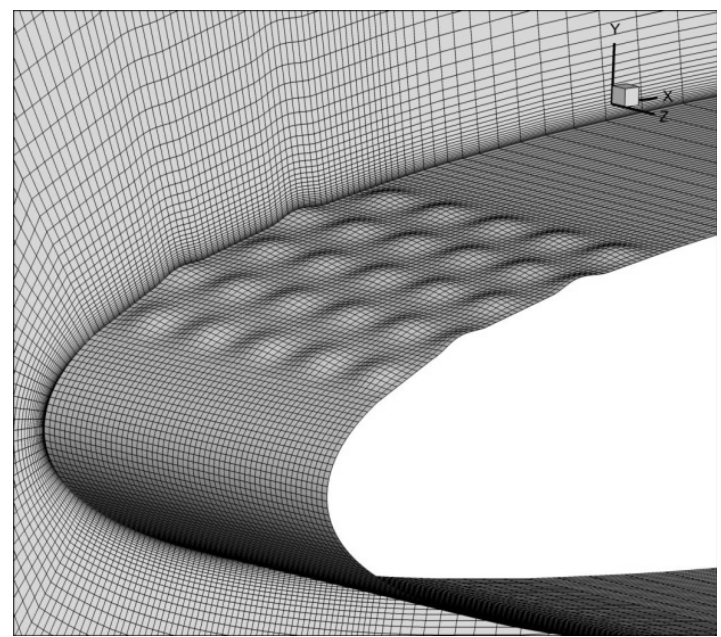

(b)

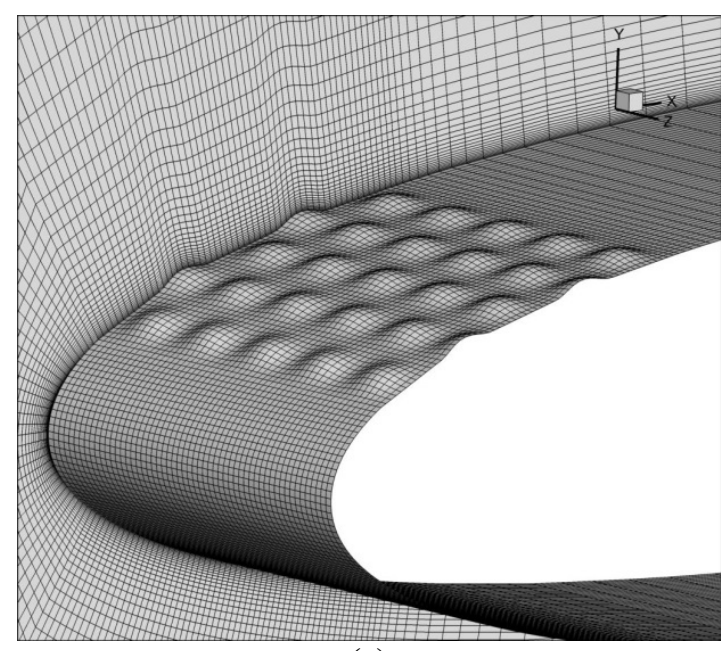

(c)

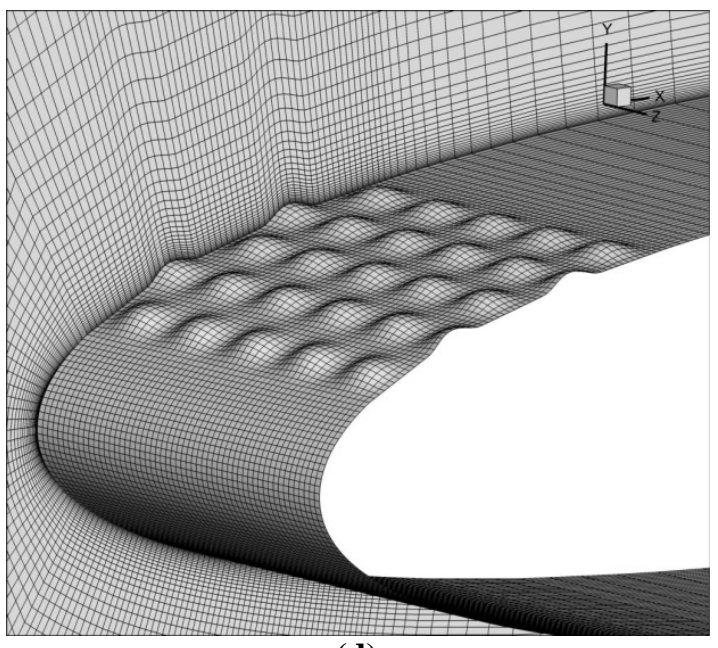

(d)

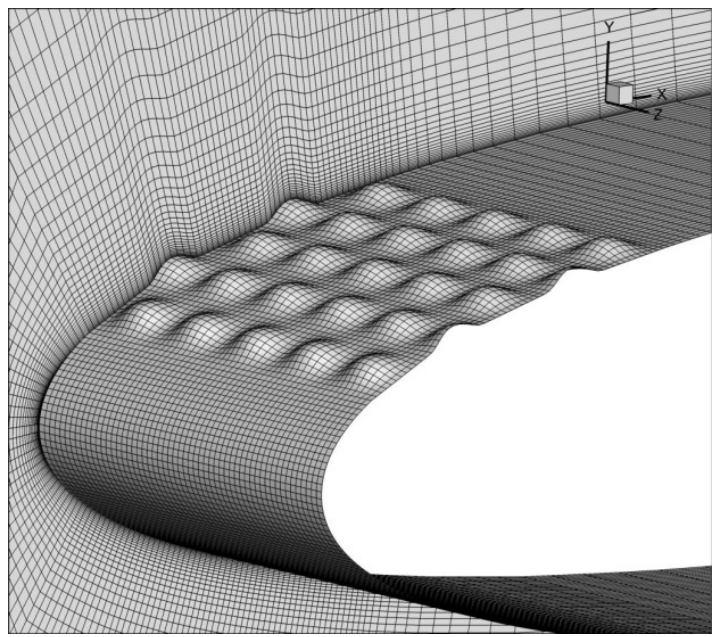

(e)

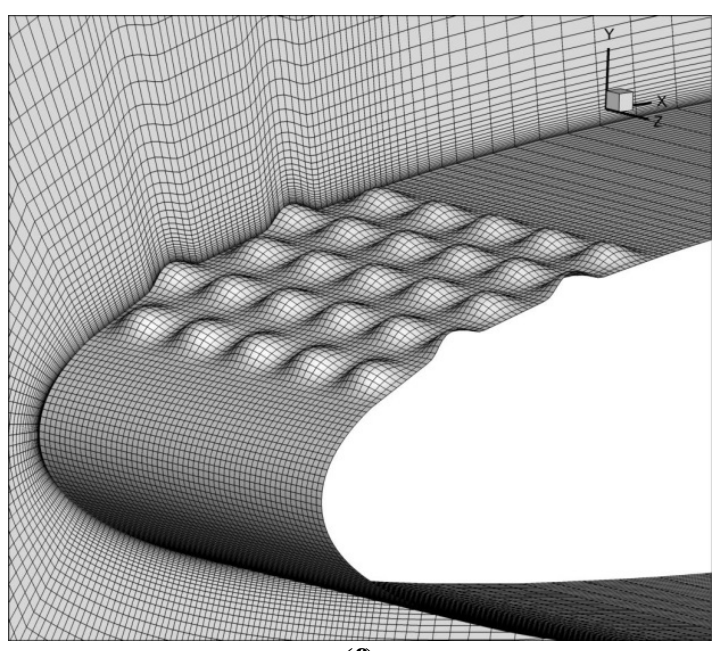

(f)

Figure 3.16 Half a cycle of three-dimensional dynamic roughness elements, starting from a clean surface (a) and reaching its maximum amplitude (f). 


\subsection{Two-Dimensional Simulations}

As in the experimental analysis, attempts will be made to answer the same three questions posed previously:

- How does the LEV development change with respect to Reynolds number?

- How does the LEV development change with respect to reduced oscillation frequency?

- Can dynamic roughness alter the development of the LEV?

- Can a relationship between LEV delay and dynamic roughness characteristics be quantitatively shown?

A consistent repeatable process was developed in regards to running CFD analysis on a rapidly pitching airfoil. At each Reynolds number studied, simulations first were run at zero angle of attack for ten domain flow through times, which is the time it takes for a particle to travel through the entire domain. This result was then used as the initial solution for the pitching case. In regards to the initial question about Reynolds number dependence, computational results seem to agree with established research as well as the PIV results. As shown in Figure 3.18 there are slight differences between each case, but in general there is not a vast difference in vortex development over this range of Reynolds number. These differences can also be explained by the shedding of vortices in the chord-wise direction, as discussed in the experimental section above. The simulations indicate vortices are shed from the LEV downstream, with reattachment occurring after a vortex is shed. It is important to remind the reader that the dynamic roughness height is sub-boundary layer height. To illustrate this, Figure 3.17 show contours of vorticity of the pitching airfoil just before LEV development with the dynamic roughness element at its maximum height. It is easy to see that the maximum height of the dynamic roughness is less than the height of the boundary layer at that point on the airfoil. 


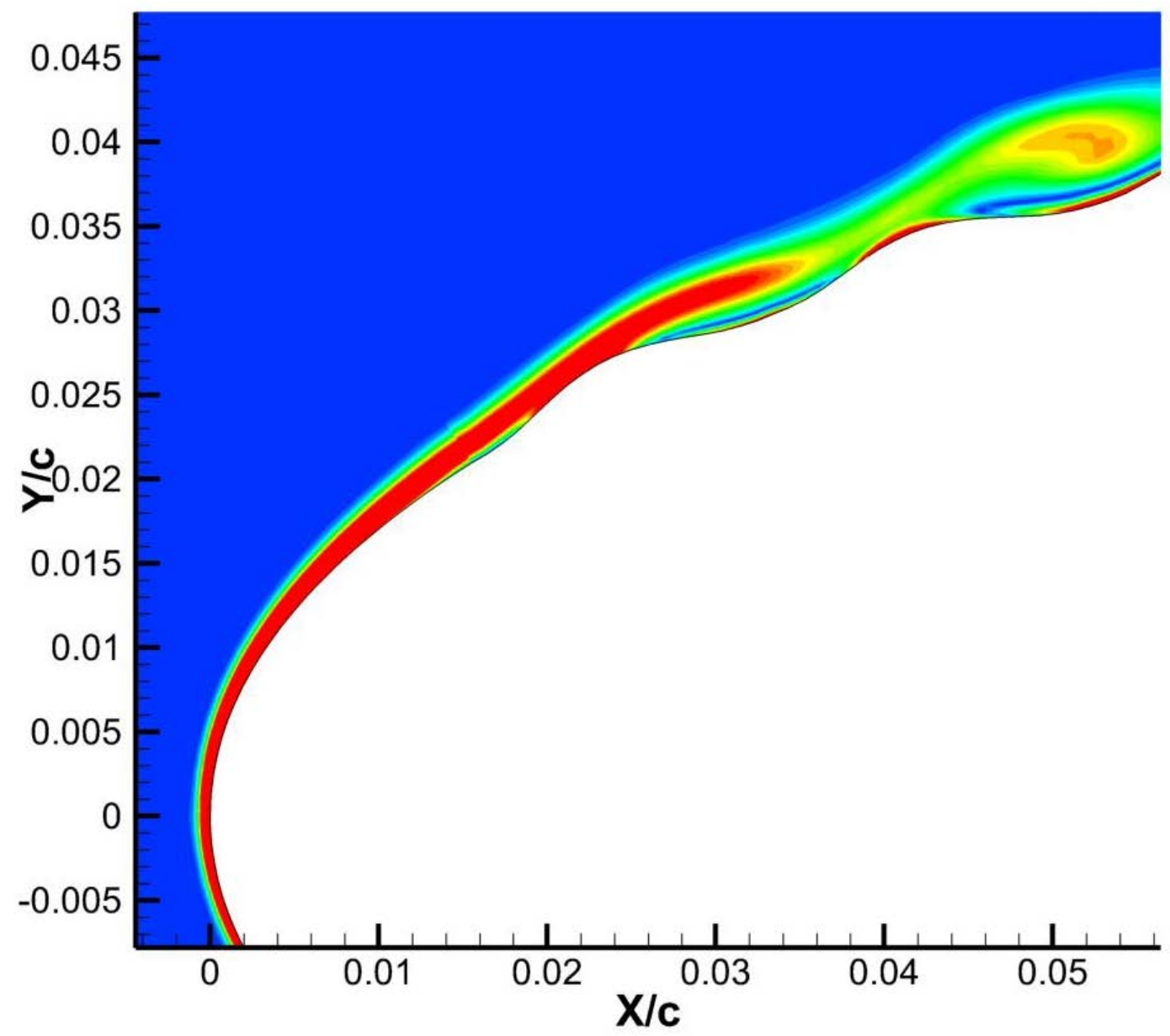

Figure 3.17 Contour plot of vorticity magnitude for a pitching airfoil just before LEV development with maximum DR element amplitude to highlight the sub-boundary layer nature of the dynamic roughness. 

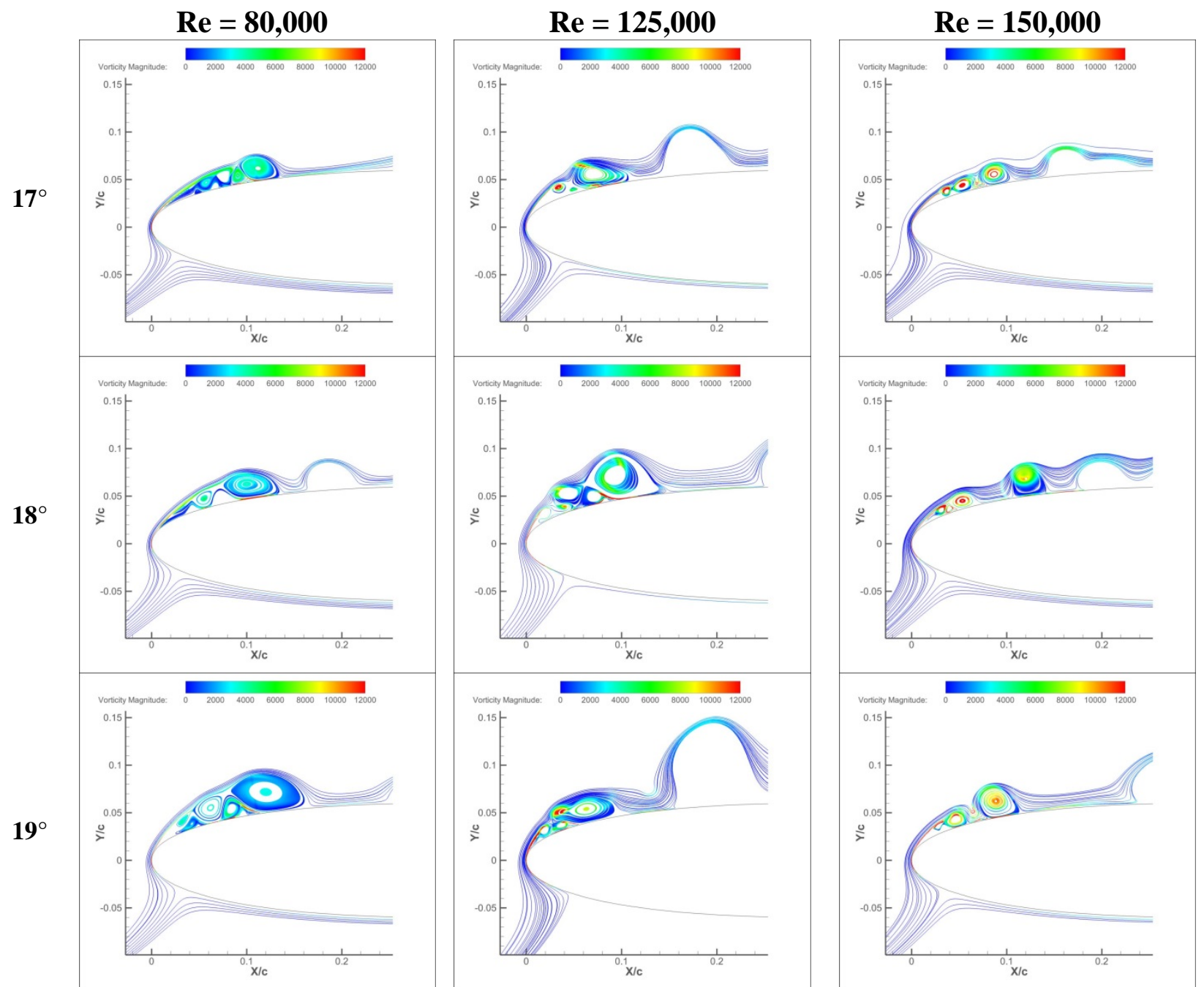

Figure 3.18 Streamline plots colored by vorticity magnitude of the variation in LEV at different Reynolds numbers while reduced frequency is held constant.

In terms of LEV development and its dependence on the reduced frequency, the computational results give similar evidence as the PIV analysis. It is clear that as reduced frequency is increased, LEV development is delayed to higher angles of attack. 

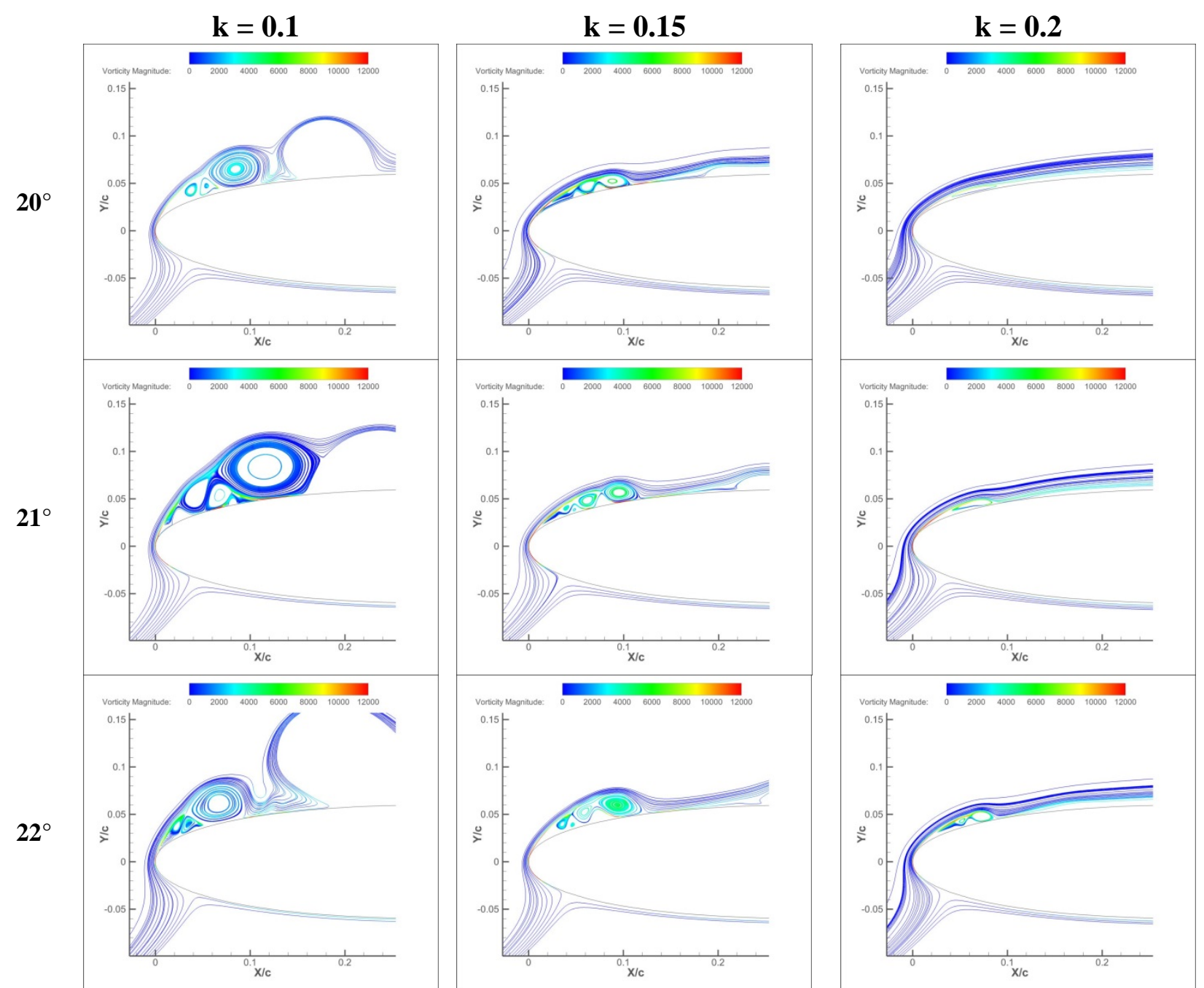

Figure 3.19 Streamline plots colored by vorticity magnitude at three different reduced frequency values.

As in the PIV experiment, a wide variety of flow conditions and dynamic roughness settings were evaluated to document the effects on LEV development. It was found that dynamic roughness becomes more effective at delaying LEV development as the Reynolds number increases. In fact, at lower Reynolds number the dynamic roughness seems to hasten LEV development. An example of this is shown in Figure 3.20 at a Reynolds number of 10,000. As is shown in the figure, LEV development is hastened by developing at a lower angle of attack as opposed to the clean airfoil. Although the major objective of this study was to hopefully delay LEV development, encouraging LEV development may also be beneficial in certain applications. For this case the dynamic roughness was actuated at a frequency of $120 \mathrm{~Hz}$ and amplitude of $10 \%$ of dynamic roughness element diameter. It should be noted that this frequency of actuation is above the capabilities of the current experimental dynamic roughness actuation mechanism. 


\section{Clean}

$22^{\circ}$

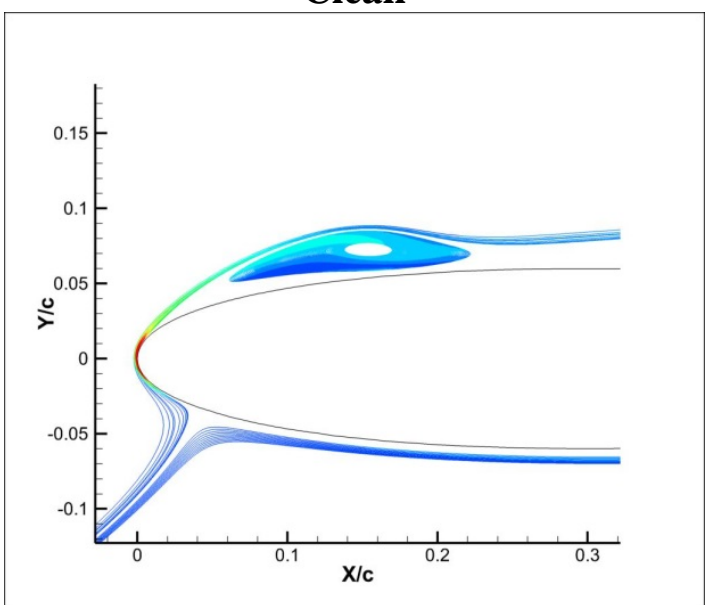

$23.5^{\circ}$

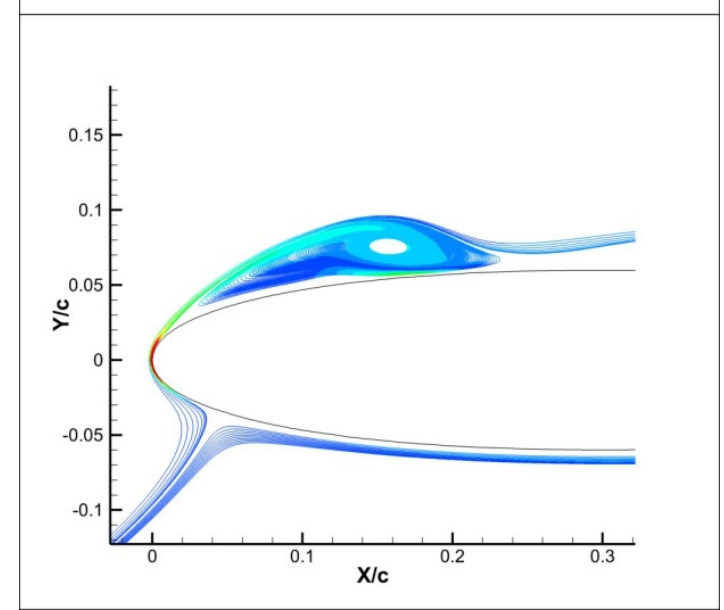

$25^{\circ}$

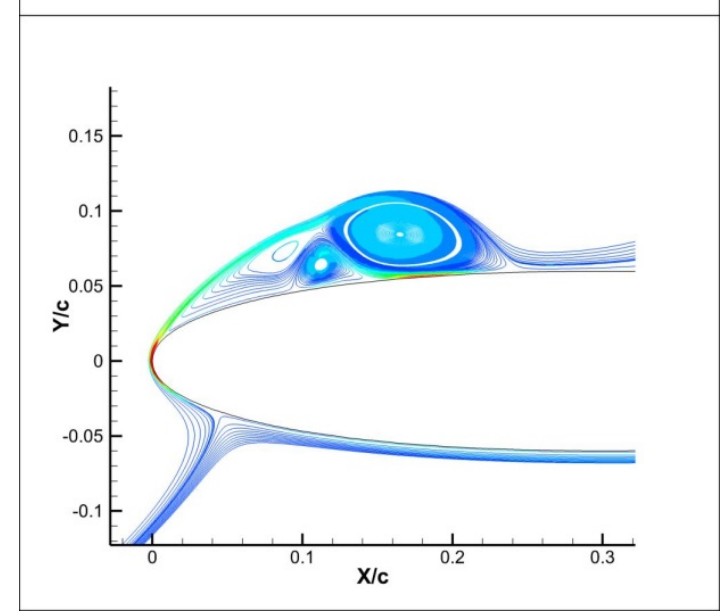

Dynamic Roughness
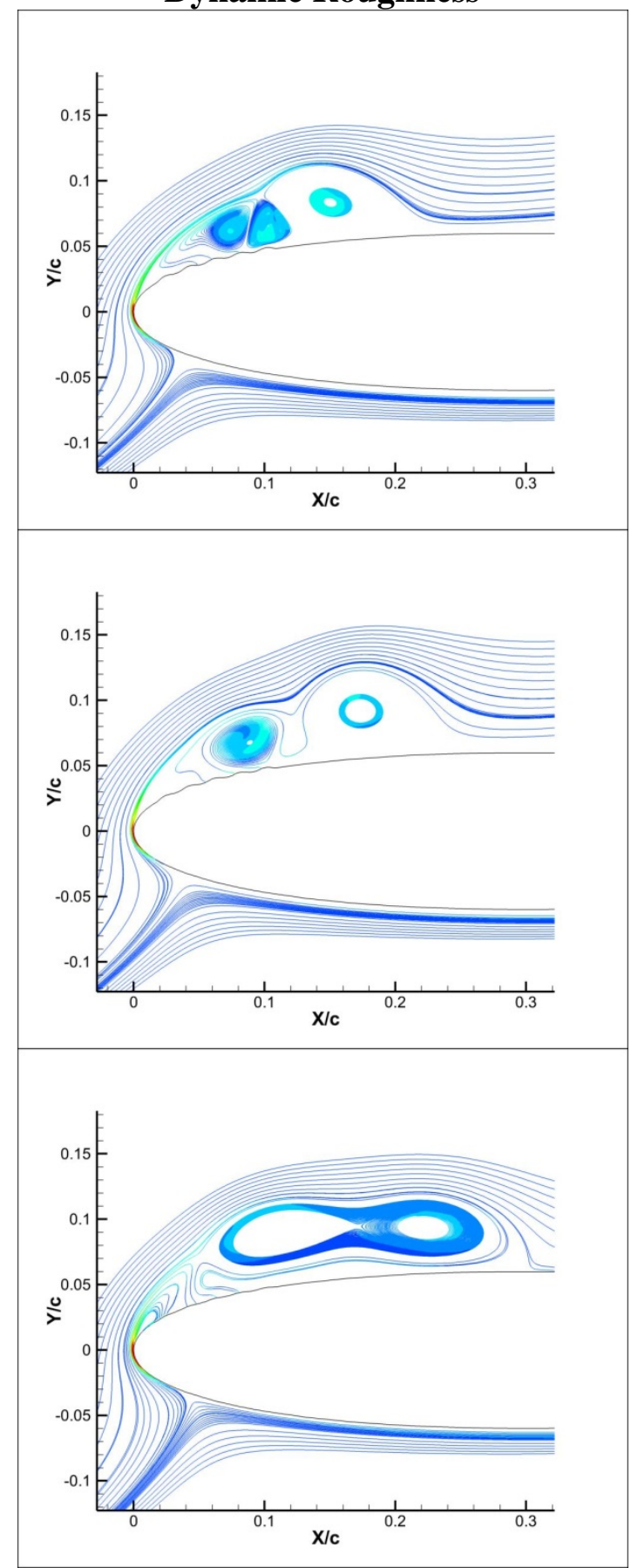

Figure 3.20

Streamlines colored by vorticity magnitude for an airfoil undergoing dynamic stall at $\mathbf{R e}=$ 10,000 without and with dynamic roughness actuation, respectively. 
A more positive effect, or an effect in which LEV development is starting to delay, occurs around $\operatorname{Re}=80,000$ as shown in Figure 3.21. The frequency of actuation could be reduced to 90 $\mathrm{Hz}$ and no significant change in the ability to delay LEV development was noticed. Most simulations were performed at this frequency since it was most effective in the experimental analysis.

$14.75^{\circ}$

$16.6^{\circ}$
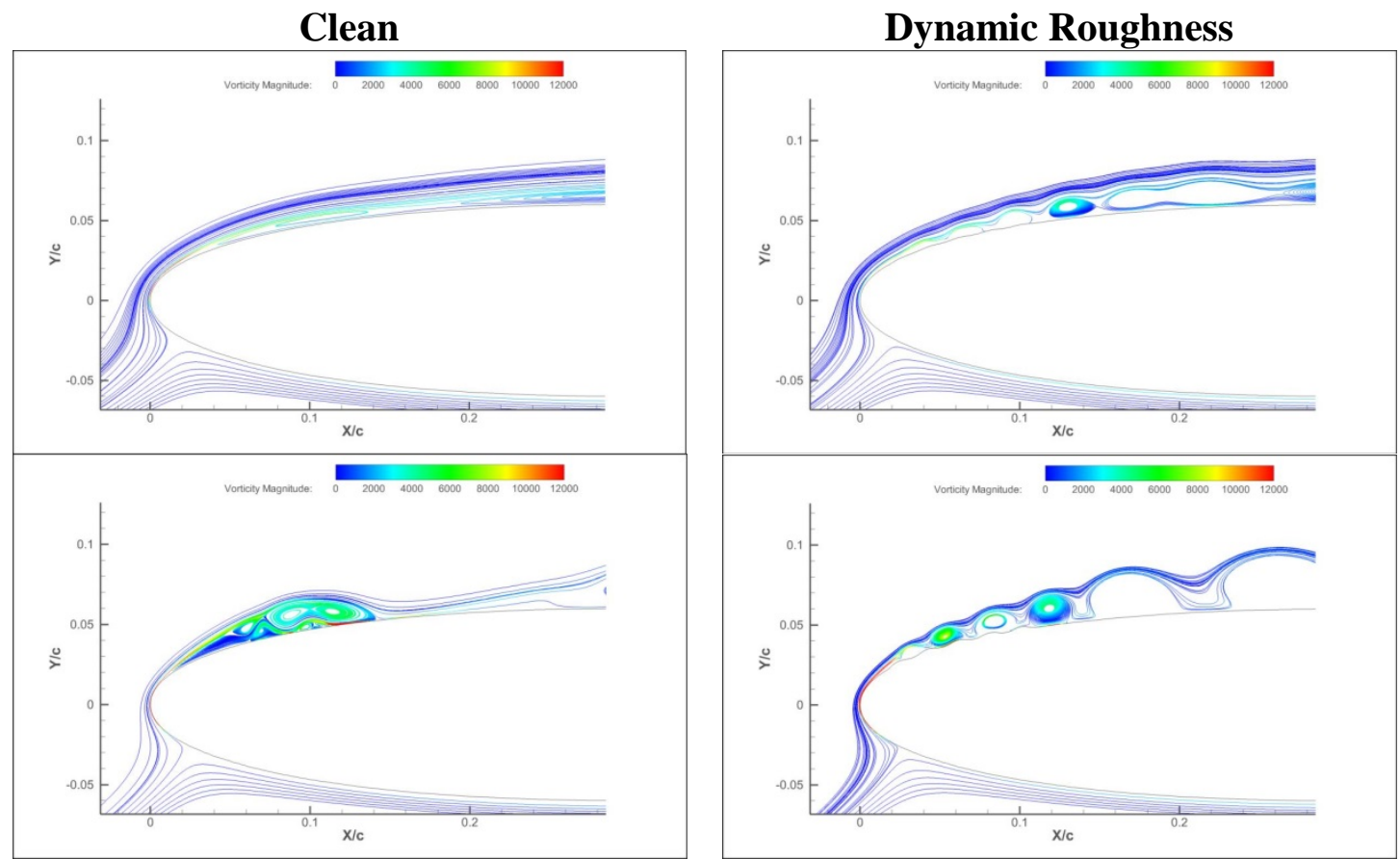

Figure 3.21 Streamlines colored by vorticity magnitude for an airfoil undergoing dynamic stall at $\mathrm{Re}=$ 80,000 without and with dynamic roughness actuation, respectively.

As Reynolds number is increased the ability for dynamic roughness to delay LEV formation increases as well. Since the laminar solver is used, simulations were not performed with a flow condition with a Reynolds number larger than 150,000. Previous research has indicated that some downstream vortex formation may be an artifact of utilizing the laminar solver [9]. To limit the formation of these vortices care was taken to try to remain in a laminar flow regime. 


\section{Clean}

$13.8^{\circ}$

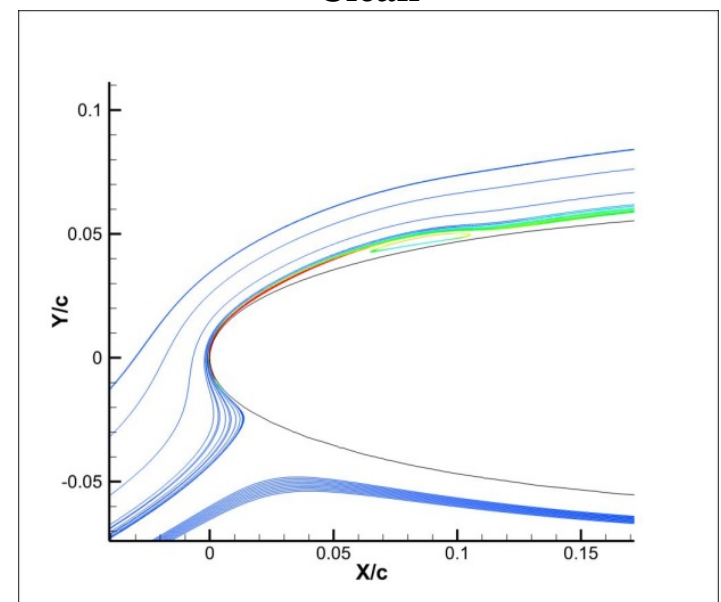

$15.2^{\circ}$

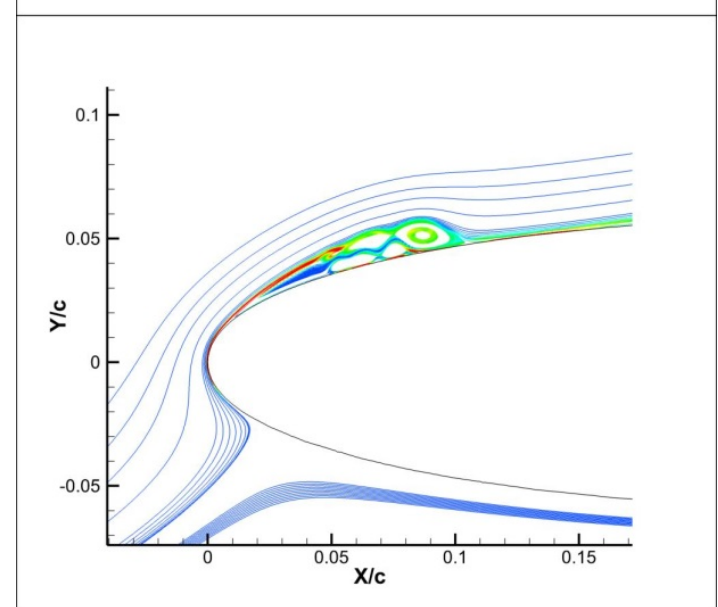

$16.6^{\circ}$

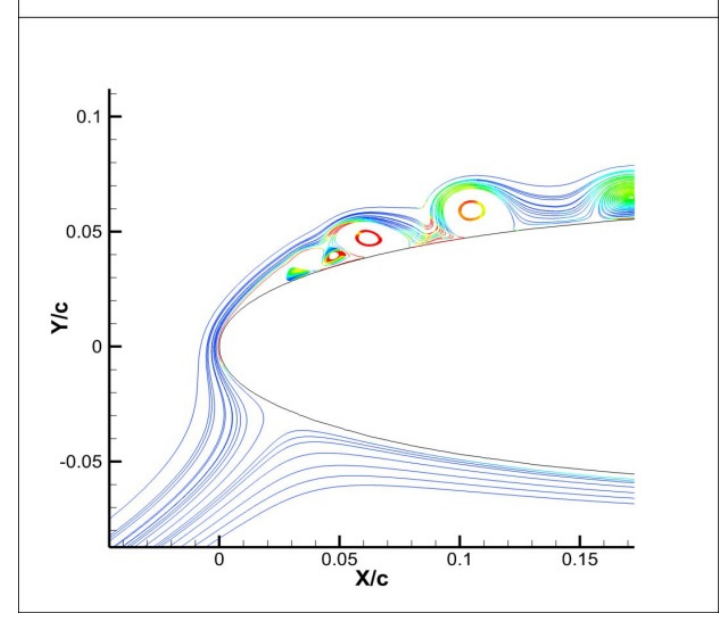

Dynamic Roughness
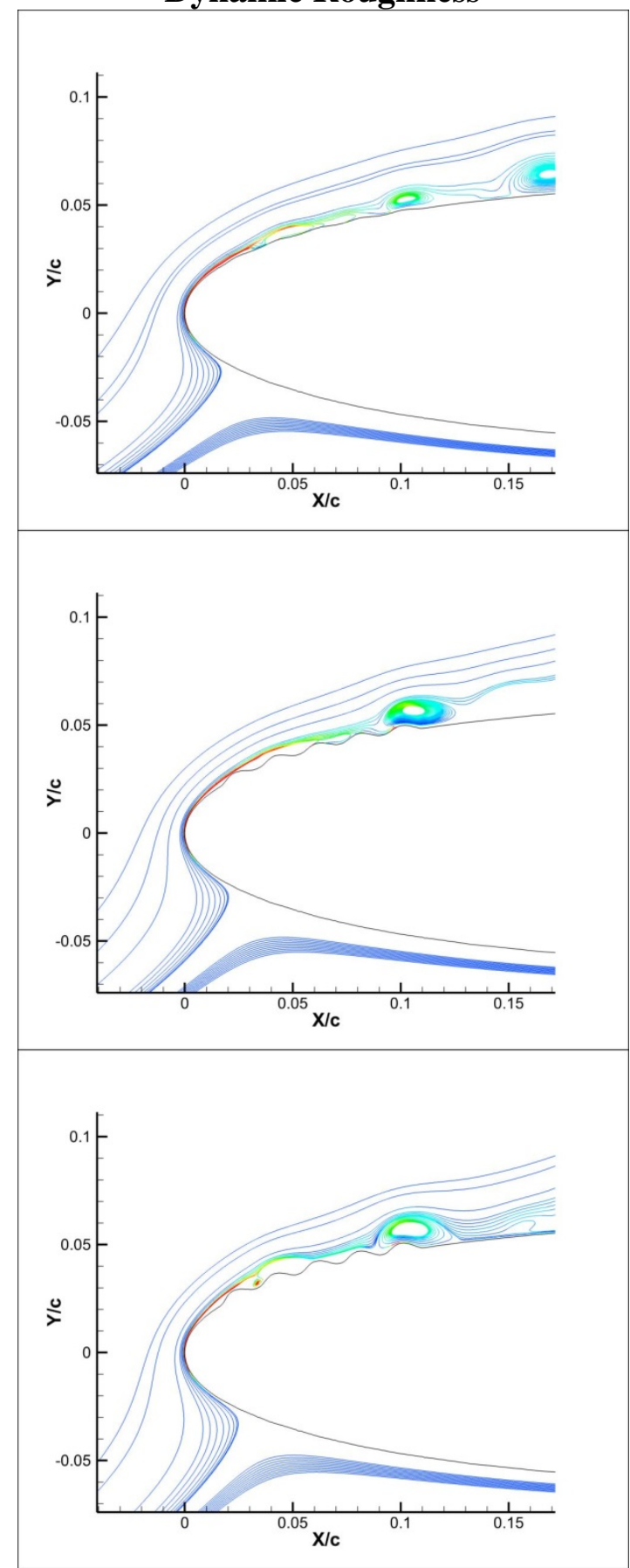

Figure 3.22

Streamlines colored by vorticity magnitude for an airfoil undergoing dynamic stall at $\mathbf{R e}=$ 150,000 without and with dynamic roughness actuation, respectively. 
To study the effect of amplitude, the previous case of $\mathrm{Re}=150,000, \mathrm{k}=0.1$, and actuation frequency $=90 \mathrm{~Hz}$ was run with an amplitude half of its current size. The results show that even at half the amplitude, LEV development was delayed. The LEV development was not as delayed as with the larger amplitude, but this shows the robustness of dynamic roughness's ability to delay LEV formation, and perhaps its ability to function at off design conditions. 

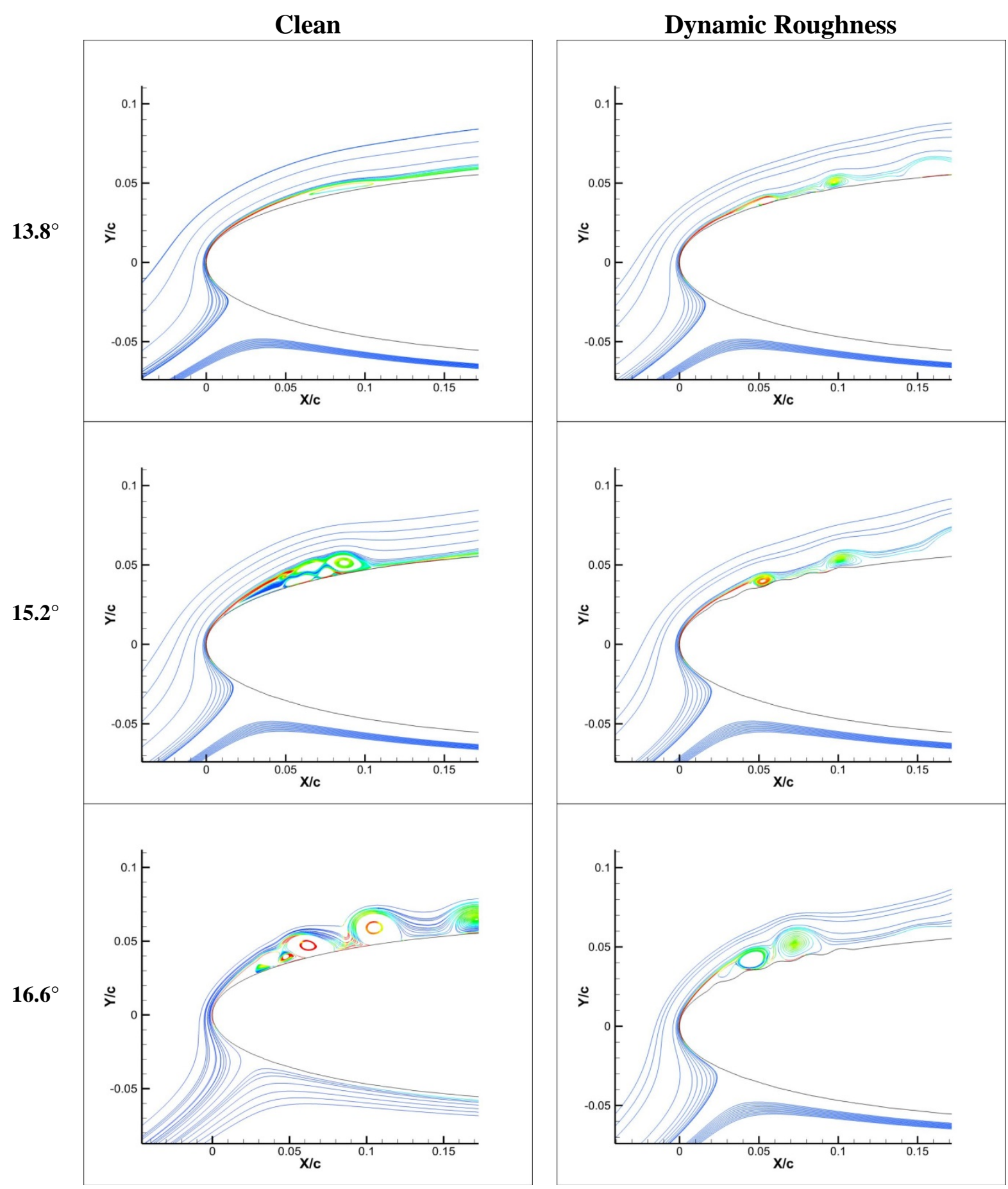

Figure 3.23 Streamlines colored by vorticity magnitude for an airfoil undergoing dynamic stall at $\operatorname{Re}=$ 150,000 without and with dynamic roughness actuation, respectively. The amplitude of the dynamic roughness is half as in the previous figure. 
If frequency is reduced to $30 \mathrm{~Hz}$ the effectiveness of dynamic roughness greatly degrades. Even if the amplitude is increased, this has no effect on LEV delay. Once the amplitude reaches a critical maximum it tends to hasten LEV development. Figure 3.24 has the same flow conditions as previous results, but the frequency of actuation has been reduced to $30 \mathrm{~Hz}$. One can clearly see that LEV development is on par with that of the clean airfoil.
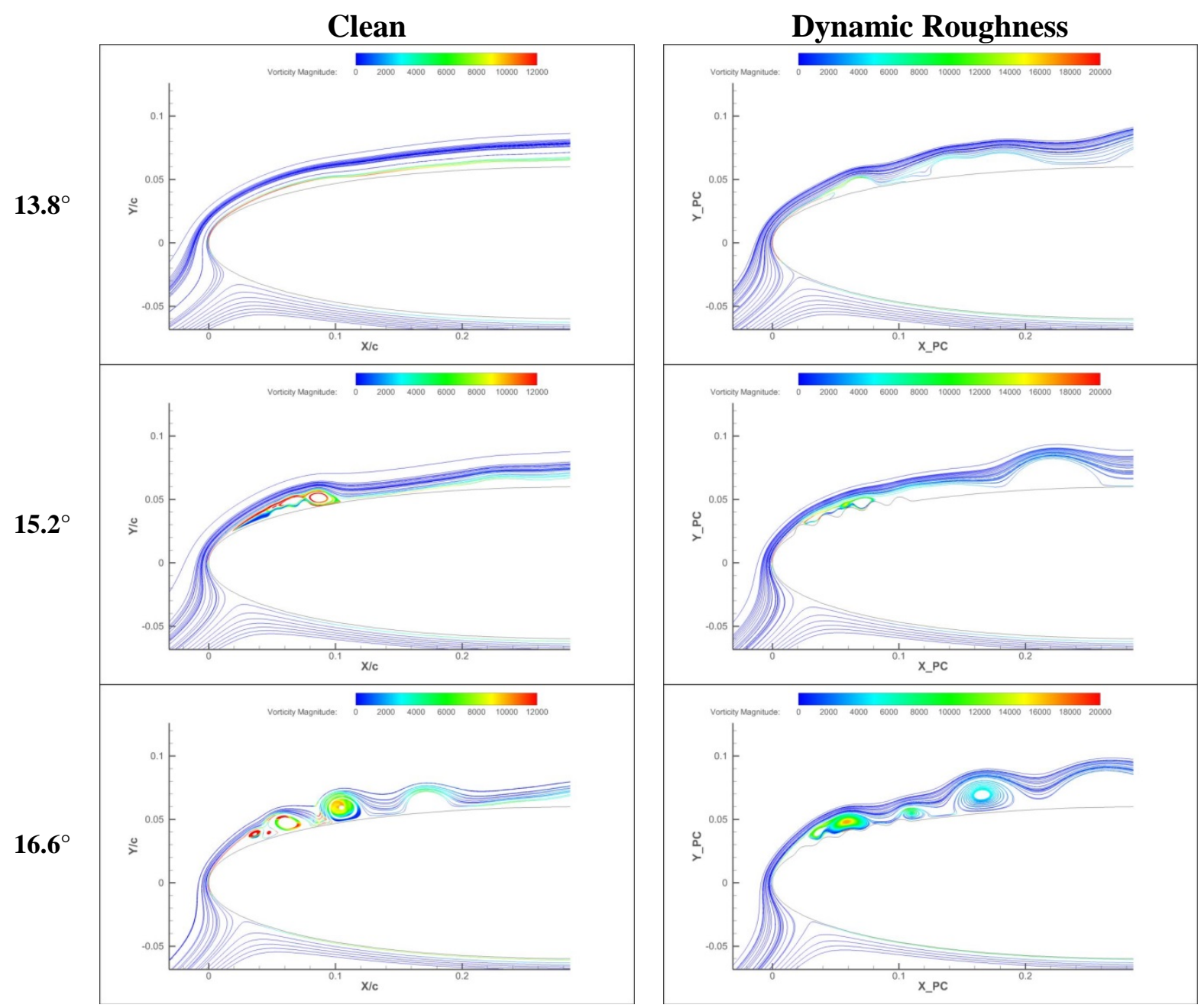

Figure 3.24 Streamlines colored by vorticity magnitude for an airfoil undergoing dynamic stall at $\operatorname{Re}=$ 150,000 without and with dynamic roughness actuation, respectively. Frequency of dynamic roughness actuation reduced to $30 \mathrm{~Hz}$ while amplitude is unchanged from previous results.

One of the most significant discoveries using computational methods was the fact that there appears to be a strict dependence on dynamic roughness actuation frequency and there is not a minimum frequency of actuation "threshold" as is the case in the laminar separation bubble for an airfoil at static angle of attack [5] [6]. This phenomenon is displayed in Figure 3.25. As 
shown, three different dynamic roughness actuation frequencies are were evaluated for the effectiveness in delaying LEV development. Reynolds number was held constant at 150,000 for all cases, as was reduced frequency $(\mathrm{k}=0.1)$, and dynamic roughness actuation amplitude $(10 \%$ roughness element diameter). It is clear that an actuation frequency of $60 \mathrm{~Hz}$ provides the most significant delay in LEV development.
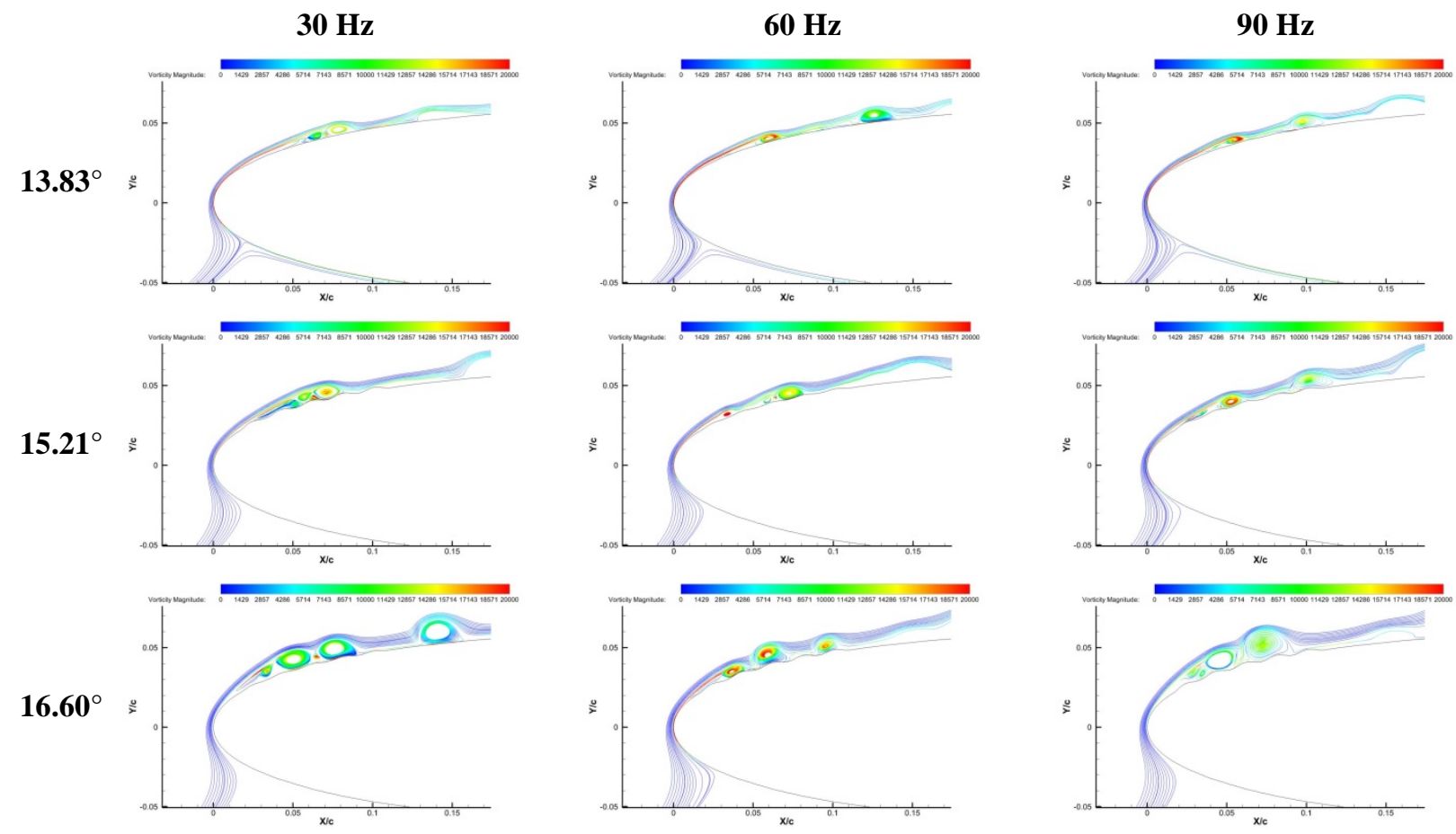

Figure 3.25 Streamlines colored by vorticity magnitude for an airfoil undergoing dynamic stall at $\mathrm{Re}=$ 150,000 with dynamic roughness actuation at a constant amplitude $(10 \%$ roughness diameter), but with an actuation frequency varying from $30 \mathrm{~Hz}, 60 \mathrm{~Hz}$, and $90 \mathrm{~Hz}$, respectively.

An attempt was made to quantitatively investigate the relationship between LEV delay and dynamic roughness characteristics. Since such a test matrix could consist of high number of combinations regarding pitch rate, Reynolds number, actuation frequency and actuation amplitude, it was decided to use two-dimensional simulations to conduct the study. In addition, only a single reduced frequency $(k=0.1)$ and constant dynamic roughness amplitude (10\% roughness element diameter) were evaluated. Six separate cases based on Reynolds number were evaluated to find the dynamic roughness actuation frequency that produced the most significant delay in LEV development. A term to characterize the relationship between dynamic roughness actuation frequency and amplitude is introduced that is simply the product of the 
dimensional actuation frequency and the dimensional amplitude. This produces a term with units of length per time, similar to velocity. Fellow researcher Ashish Robert, Ph.D. has termed this as a “Dynamic Roughness Strength” (personal communication, May 2012). This term is then nondimensionalized by the free stream velocity and then compared to the Reynolds number. The results of this study are shown in Table 3.3.

Table 3.3 Tabulated data comparing dynamic roughness strength with Reynolds number at a single reduced frequency of $k=0.1$.

\begin{tabular}{|c|c|c|c|c|c|c|}
\hline $\begin{array}{c}\text { Reynolds } \\
\text { Number }\end{array}$ & $\mathbf{k}$ & $\begin{array}{c}\text { Free-stream } \\
\text { Velocity } \\
(\mathbf{m} / \mathbf{s})\end{array}$ & $\begin{array}{c}\text { DR Frequency } \\
\mathbf{( H z}\end{array}$ & $\begin{array}{c}\text { DR Amplitude } \\
\mathbf{( m )}\end{array}$ & $\begin{array}{c}\text { DR Strength } \\
\mathbf{( m / \mathbf { s } )}\end{array}$ & $\begin{array}{c}\text { Non-dimensional } \\
\text { DR Strength }\end{array}$ \\
\hline 50,000 & 0.1 & 2.5 & 60 & $5.84 \times 10^{-4}$ & $3.51 \times 10^{-2}$ & $1.40 \times 10^{-2}$ \\
\hline 60,000 & 0.1 & 3.0 & 60 & $5.84 \times 10^{-4}$ & $3.51 \times 10^{-2}$ & $1.17 \times 10^{-2}$ \\
\hline 80,000 & 0.1 & 4.0 & 60 & $5.84 \times 10^{-4}$ & $3.51 \times 10^{-2}$ & $8.76 \times 10^{-3}$ \\
\hline 100,000 & 0.1 & 5.0 & 60 & $5.84 \times 10^{-4}$ & $3.51 \times 10^{-2}$ & $7.00 \times 10-3$ \\
\hline 125,000 & 0.1 & 6.25 & 60 & $5.84 \times 10^{-4}$ & $3.51 \times 10^{-2}$ & $5.60 \times 10^{-3}$ \\
\hline 150,000 & 0.1 & 7.5 & 60 & $5.84 \times 10^{-4}$ & $3.51 \times 10^{-2}$ & $4.67 \times 10^{-3}$ \\
\hline
\end{tabular}

It is interesting to note that for all the cases in the table shown above a dynamic roughness actuation frequency of $60 \mathrm{~Hz}$ produced the most significant delay in LEV development. This leads the author to believe the optimum dynamic roughness frequency of actuation is more coupled with the pitching rate than with flow velocity. 


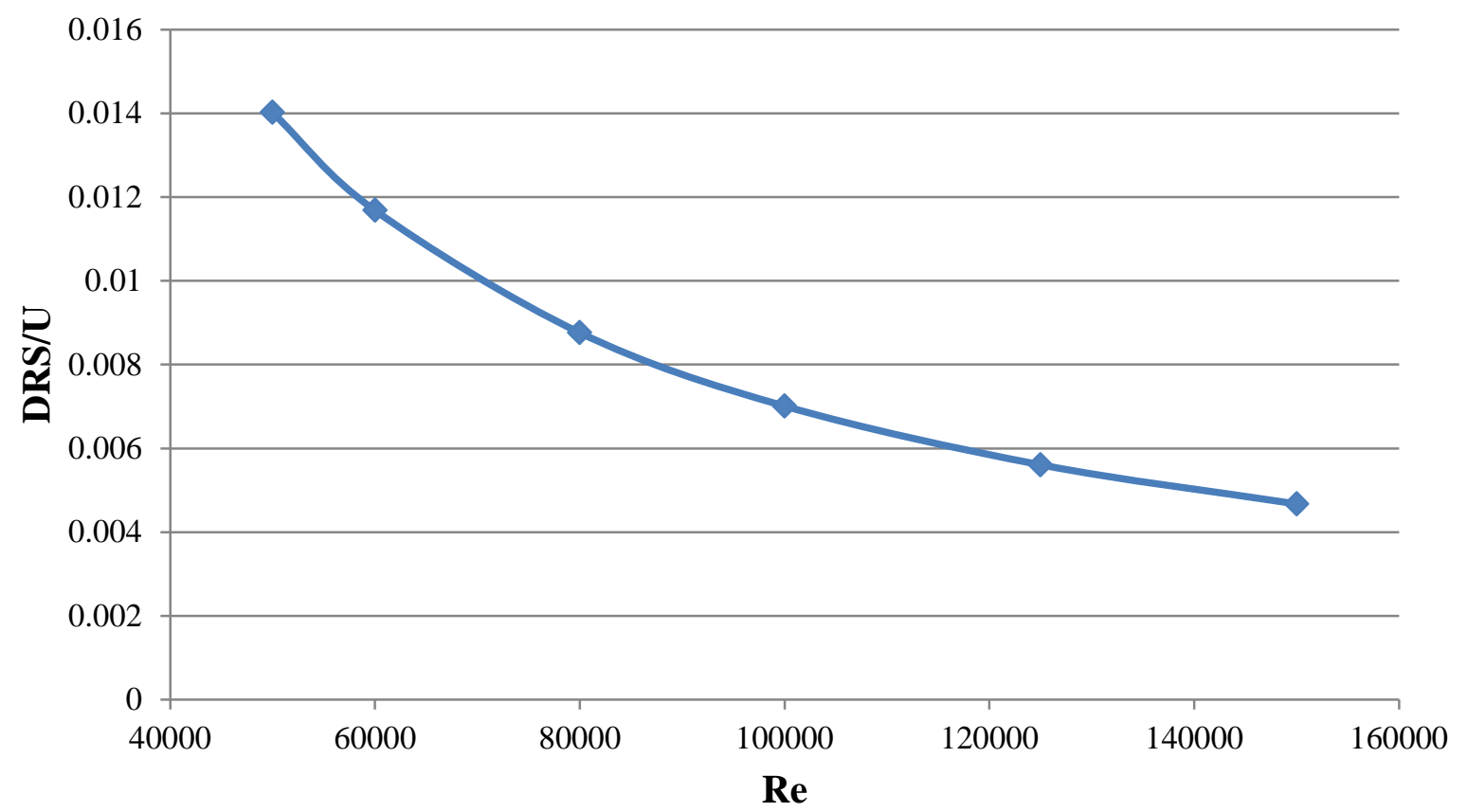

Figure 3.26 Plot of non-dimensional dynamic roughness strength versus Reynolds number at a pitching rate with a reduced frequency of 0.1 .

Figure 3.26 is the plot of the non-dimensionalized dynamic roughness strength compared to Reynolds number. One can see the trend for the curve to exponentially increase as Reynolds number decreases. This agrees with the computational observations that it is harder to delay LEV development at lower Reynolds number, most likely due to the larger boundary layer height near the leading edge. If the same data is plotted on a log-log scale the curve appears as a straight line and therefore indicates a monomial relationship between non-dimensional dynamic roughness strength and Reynolds number. Another important observation to note about the plot shown in Figure 3.26 is that it appears the curve is starting to reach its asymptotic value as Reynolds number is increasing. This may give the appearance that a fixed value of nondimensional dynamic roughness strength would be suitable for those relatively higher Reynolds numbers, but most likely the onset of turbulent separation and reattachment will alter the required dynamic roughness characteristics. 


\subsection{Three-Dimensional Simulations}

Three-dimensional simulations were conducted to account for any three-dimensional aspects of dynamic stall that are neglected when conducting two-dimensional analysis. It would also be worthwhile to know if two-dimensional simulations can adequately predict dynamic roughness effectiveness on delaying LEV development. The three dimensional mesh is similar to the twodimensional mesh in that it consists of a C-grid blocking scheme with all structured elements in the domain. As in the two-dimensional mesh, the domain extends to 15 chord lengths upstream and normal to the airfoil surface and 25 chord lengths aft of the airfoil. A uniform spacing of 0.0018c over each dynamic roughness element is employed. The dynamic roughness elements extents are $0.02 \mathrm{c}$ in chord-wise length. The dynamic roughness region starts at $0.0156 \mathrm{c}$ and extends to 0.111c. A hyperbolic stretching scheme is employed over the remaining airfoil to the trailing edge to coarsen the node distribution at about mid-chord, and then condense again near the trailing edge. This smallest spacing over this region is $0.0018 \mathrm{c}$ and it reaches a maximum spacing of 0.0338c. A geometric spacing is also employed normal to the airfoil surface to condense nodes within the boundary layer, but coarsen as distance away from the airfoil surface increases. The minimum spacing near the airfoil surface is $0.0002 \mathrm{c}$ and stretches to $0.42 \mathrm{c}$ at a distance 3.4c normal to the airfoil surface. The spanwise spacing is kept uniform and the same as the spacing over the dynamic roughness elements. This ensures accurate shape representation as the dynamic roughness elements are actuated. The total span is $0.1 c$, which contains five dynamic roughness elements. This produces a mesh with just over 1.8 million nodes. It was desired to keep the node count to below 2 million for computational considerations. All threedimensional simulations were conducted at $\mathrm{Re}=150,000$, since this showed adequate delay of LEV development in PIV results.

Before presenting dynamic roughness results, first clean airfoil results will be compared from two-dimensional simulations and three-dimensional simulations. Streamlines shaded in regards to vorticity magnitude are presented in Figure 3.27. As shown in the figure, a similar vortex structure is developed in both cases. Two major differences exist between each case. The threedimensional simulation produces the vortex at an earlier time in the pitching motion, and thus at a lower angle of attack. For example, in the figure below the angle of attack for the twodimensional case is $15.21^{\circ}$ while the three-dimensional simulation has already formed the LEV 
by $11.76^{\circ}$. In addition, the reattachment point is farther downstream in the three-dimensional case. For the two-dimensional case reattachment occurs at about $0.1 \mathrm{c}$, while in the threedimensional case the flow seems to reattach at $0.16 \mathrm{c}$.

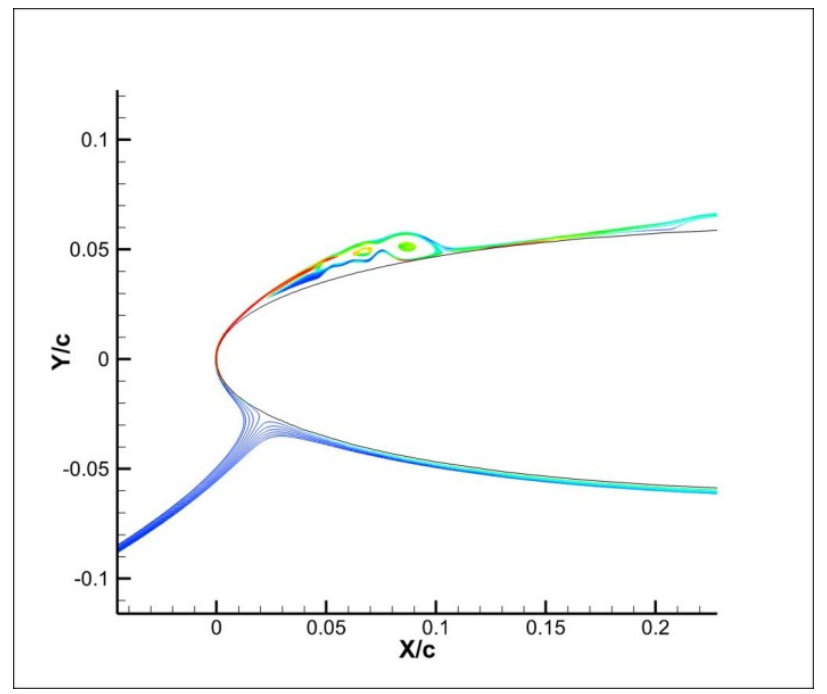

(a)

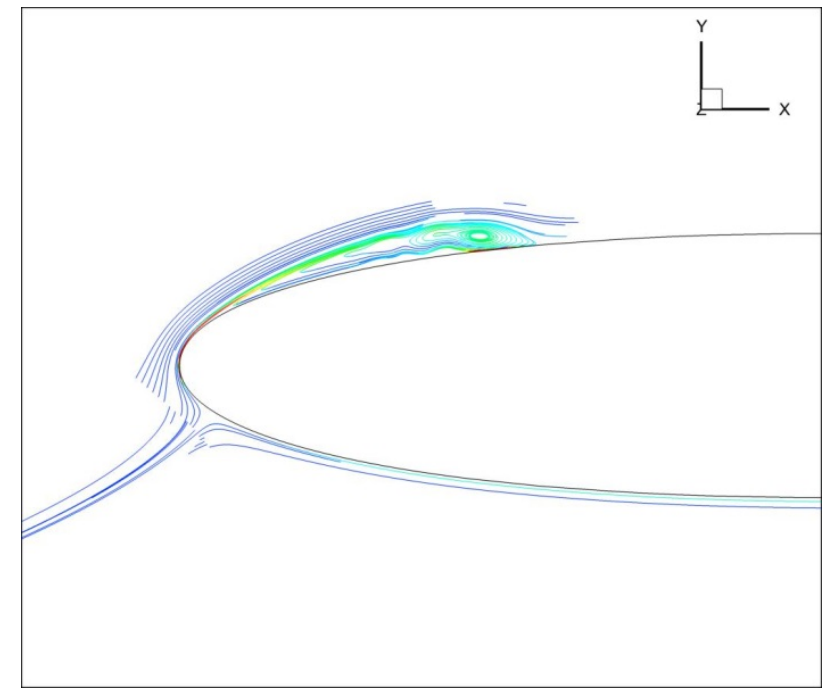

(b)

Figure 3.27 Comparison between (a) two-dimensional and (b) three-dimensional simulation results of an airfoil undergoing rapid pitching at $R e=150,000$ and $k=0.1$.

If we take a view slightly to the side of the leading edge and look down the airfoil chord, we notice a small amount of span-wise flow, as shown in Figure 3.28. This span-wise flow may dissipate some energy from the LEV to alter its reattachment point. These subtle differences may also be a result of the use of a mesh more coarse in the three-dimensional studies. The normal and tangential node spacing is slightly larger than the two-dimensional case to keep computational time manageable. 


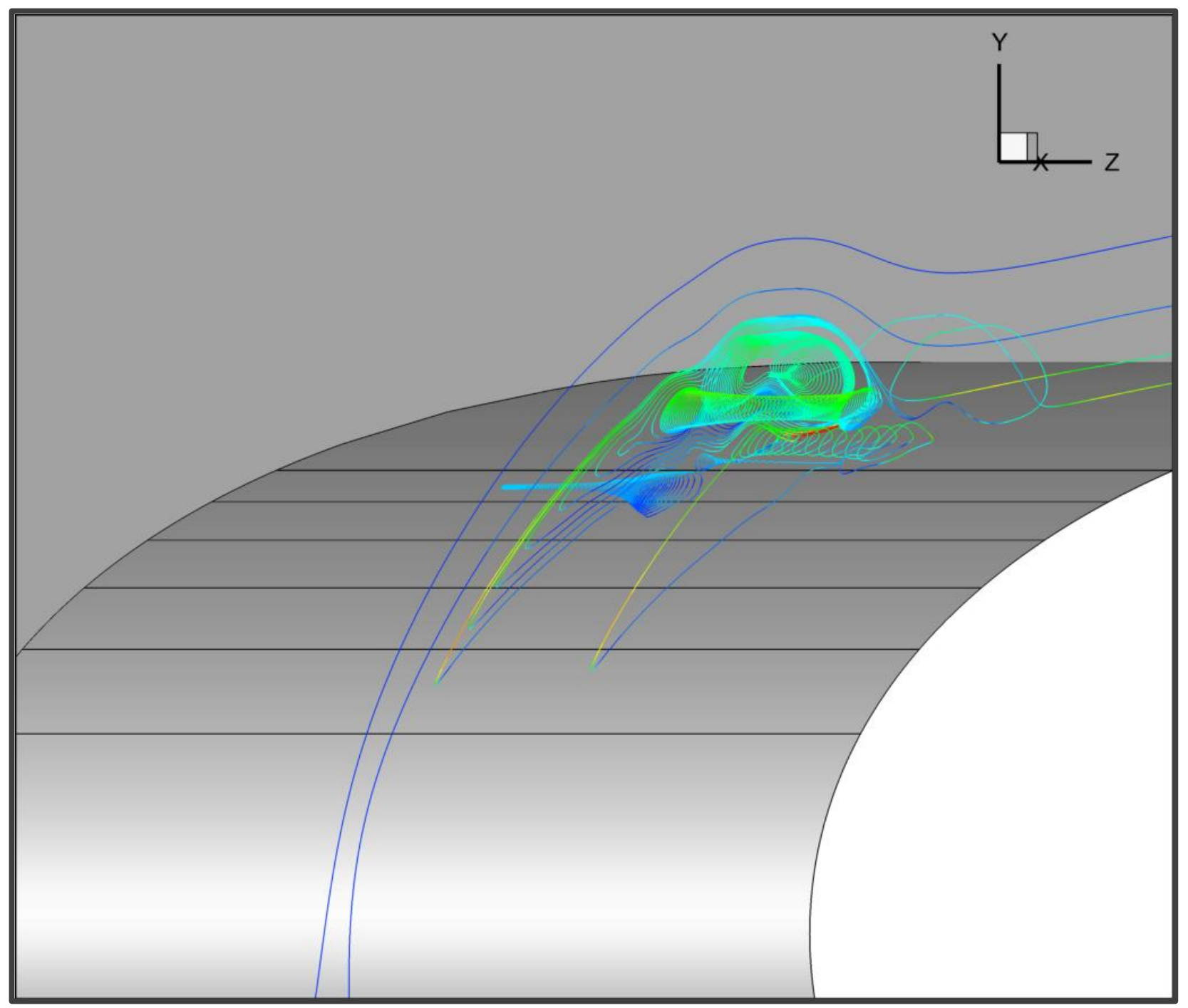

Figure 3.28 Streamlines shaded by vorticity magnitude, showing span-wise flow within the LEV region.

Delay of LEV development due to dynamic roughness is similar to the results gathered by twodimensional simulations. Figure 3.29 provides further three-dimensional validation that dynamic roughness can delay LEV formation. Even at a time with full dynamic roughness actuation, the small vortices developed by the dynamic roughness elements themselves are small in comparison to the LEV developed over the entire region in the clean case. 


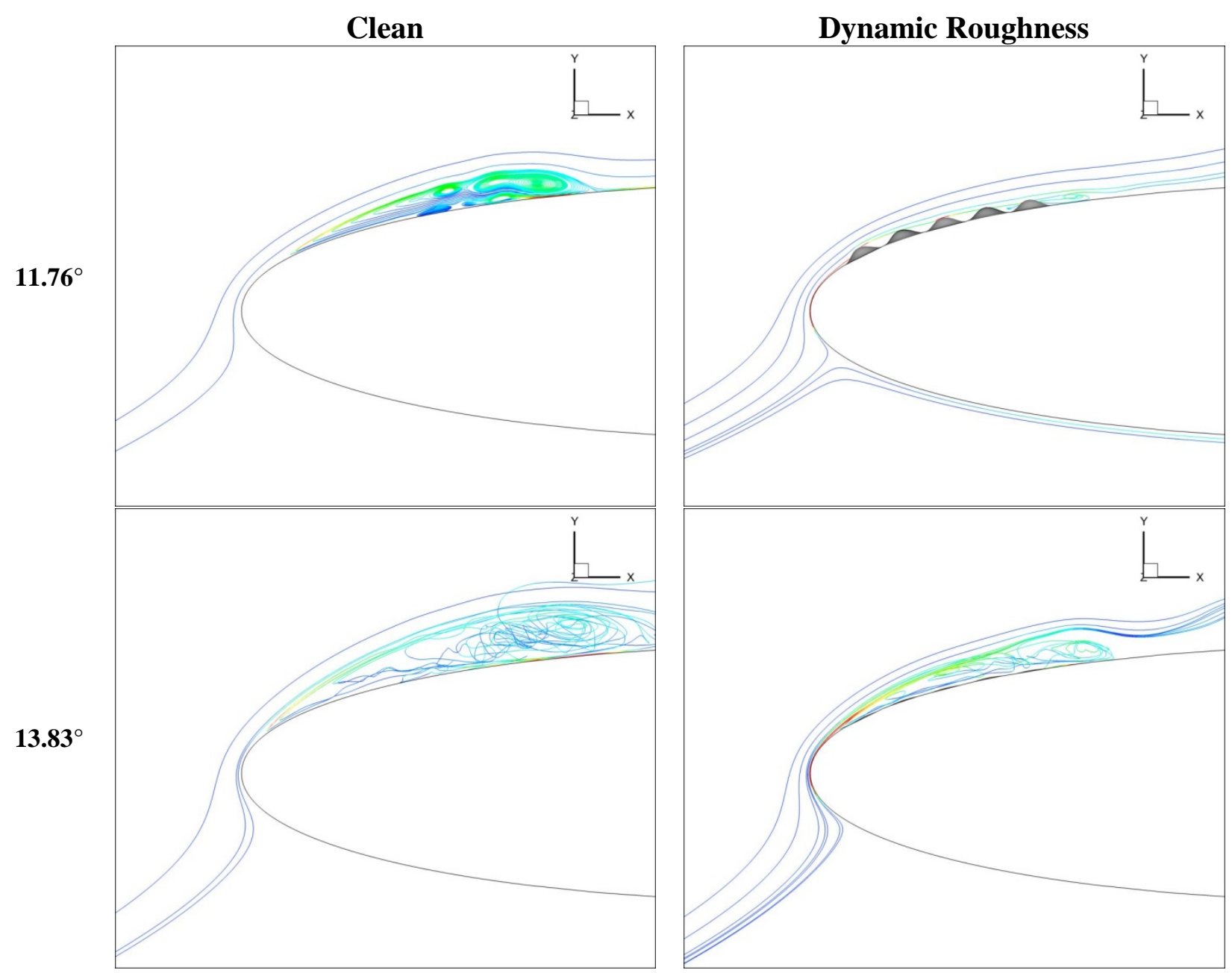

Figure 3.29 Three dimensional streamline comparison of a clean airfoil with an airfoil with actuated dynamic roughness.

An interesting plot was developed and is provided in Figure 3.30. The plot consists of an isosurface plot of a single vorticity magnitude value, contoured by the chord-wise velocity component. With this information it is interesting to see how the vorticity is altered by the dynamic roughness, and seemingly mostly by the first two rows of dynamic roughness elements. The very first row seems to develop a very short bulge region, which seems to accelerate flow between adjacent elements. This accelerated flow then creates a long bulge region that seems to remain unaltered by the aft dynamic roughness elements. 


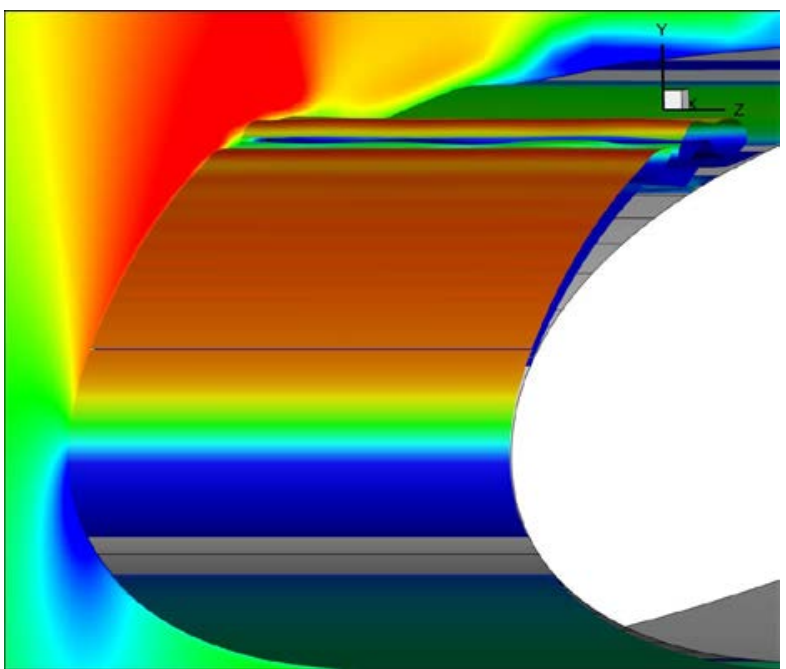

(a)

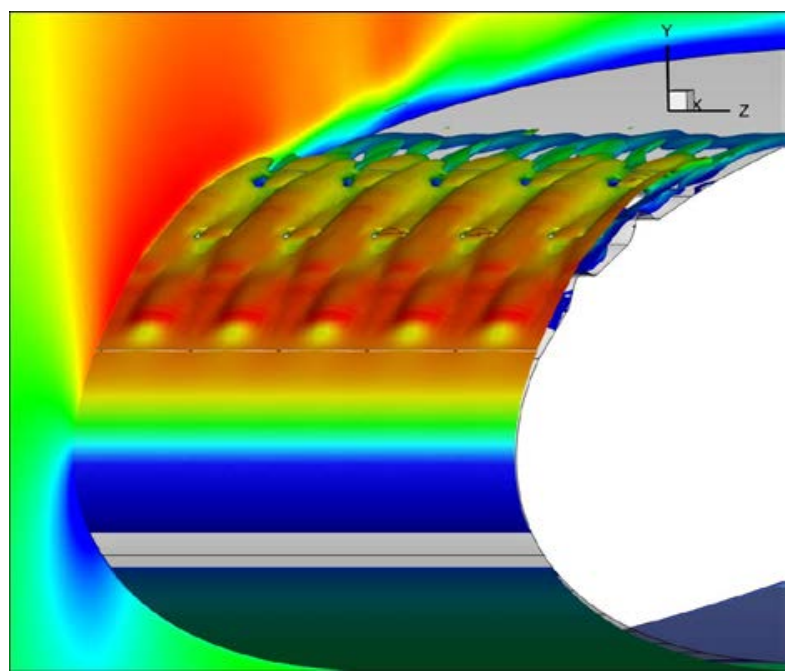

(b)

Figure 3.30 Iso-surface of a single vorticity magnitude contoured by chord-wise velocity component for a (a) clean airfoil at $11.76^{\circ}$ angle of attack and a (b) airfoil with actuated dynamic roughness at the same angle of attack.

Although slight differences between two-dimensional and three-dimensional simulations are present, the trend to delay LEV development is the same. With the ability to run simulations on faster computers, a more refined grid may be able to be employed that may bring the twodimensional and three-dimensional simulations closer together in terms of comparison of results. The main purpose of the three-dimensional simulations was to investigate any span-wise flow that is not captured by the two-dimensional simulations. Since there is very little span-wise flow this provides more validation to the results obtained with two-dimensional analysis. Nevertheless, each simulation has provided insight into the nature of the dynamic roughness as the airfoil is pitching up. Analyzing both two-dimensional and three-dimensional simulations creates a more complete picture of the flow in and around the dynamic roughness elements. 


\section{CHAPTER 4: DISCUSSION}

In the research presented, computational and experimental analysis was performed to evaluate the ability of dynamic roughness to alter the development of the leading edge vortex on a pitching airfoil undergoing dynamic stall in a low Reynolds number range of $10^{4} \leq \operatorname{Re}_{\mathrm{c}} \leq 1.5 \mathrm{x}$ $10^{5}$. Both two-dimensional and three-dimensional simulations were completed, as were both planar and stereoscopic PIV analyses. A variety of flow conditions and dynamic roughness characteristics were evaluated. With the results outlined above, dynamic roughness appears to be a good candidate for LEV flow control in certain conditions.

Both experimental and computational results provide further evidence for certain characteristics of dynamic stall that have been previously proposed. LEV development appears to be independent of Reynolds number, as long as the reduced frequency, $\mathrm{k}$, is held constant at each Reynolds number. This fact supports the use of reduced frequency as the characteristic term used to characterize the pitching motion. In contrast to Reynolds number dependency, reduced frequency greatly affects the angle of attack at which LEV development starts, as well as its growth rate. Evidence to support this was observed in both the experimental and computational studies. As reduced frequency is increased, LEV development is delayed to higher angles of attack. This change in LEV development may be related to a vortex shedding frequency change. It is a hypothesis that there is a relationship between dynamic roughness actuation frequency and vortex shedding frequency. If the vortex shedding frequency could be related to the reduced frequency, which governs the pitching motion, a relationship could be developed between dynamic roughness actuation and reduced frequency. This relationship could easily be inputted to a theoretical flow control system that could sense pitching rate (or be given a prescribed pitching rate) and in turn apply the appropriate frequency of actuation to optimally delay LEV development.

Previous work for static airfoils concluded there was a relationship between dynamic roughness actuation frequency and amplitude [5]. They concluded that if the frequency of actuation was reduced laminar separation could still be prevented, in terms of a laminar separation bubble, if the amplitude increased. This does not seem to be the case for LEV development during dynamic stall. The numerical study showed that even when amplitude was increased to about 
$30 \%$ the diameter of the dynamic roughness element, LEV delay was not established. Most likely this is an effect of the unsteadiness of dynamic stall in which frequency of actuation plays a larger role.

For completeness, a brief comparison between PIV results and CFD results is presented. Since the PIV results consisted of phase averaged calculations, much of the detail of the leading edge vortex is lost. But, the basic size and development of the LEV are fairly consistent between the two methods. Slight differences, shown in Figure 4.1, tend to include a shorter distance away from the airfoil's surface the LEV reaches and a longer distance chord-wise reattachment occurs for the PIV analysis. Most likely this aft stretching of the LEV is most likely due to the bias of shedding vortices convecting downstream. Also, the reader is reminded that the inertial terms of the governing equations are neglected in the computational studies due to the method of simulating the pitch-up maneuver.

$26^{\circ}$
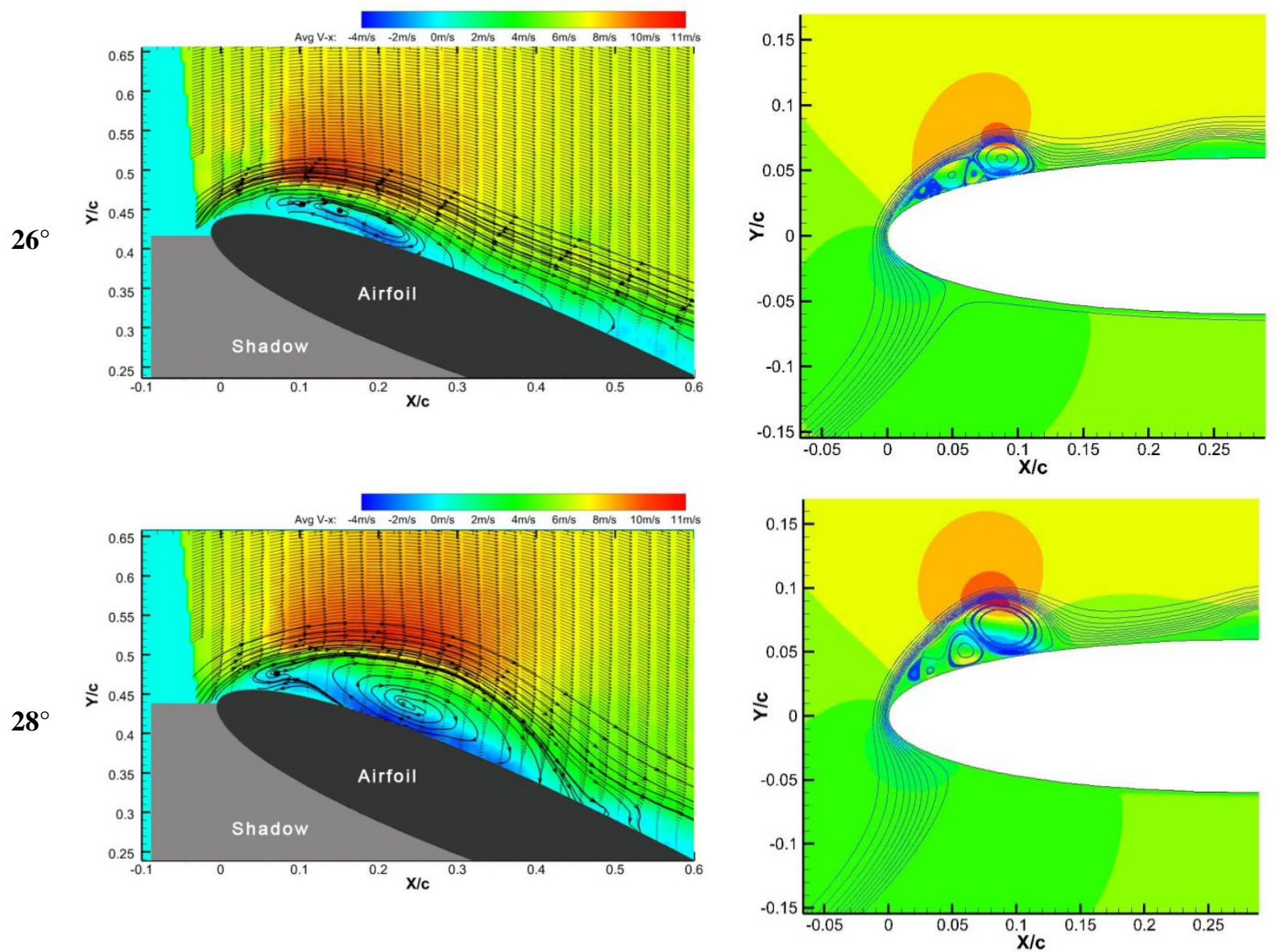

Figure 4.1 Comparison of PIV results with those collected via CFD. 
Figure 4.2 provides a comparison between phase averaged PIV results and a single instantaneous PIV vector field. There appears to be multiple recirculating regions within the LEV on the instantaneous image, which corroborates well with what is shown in literature and CFD results.

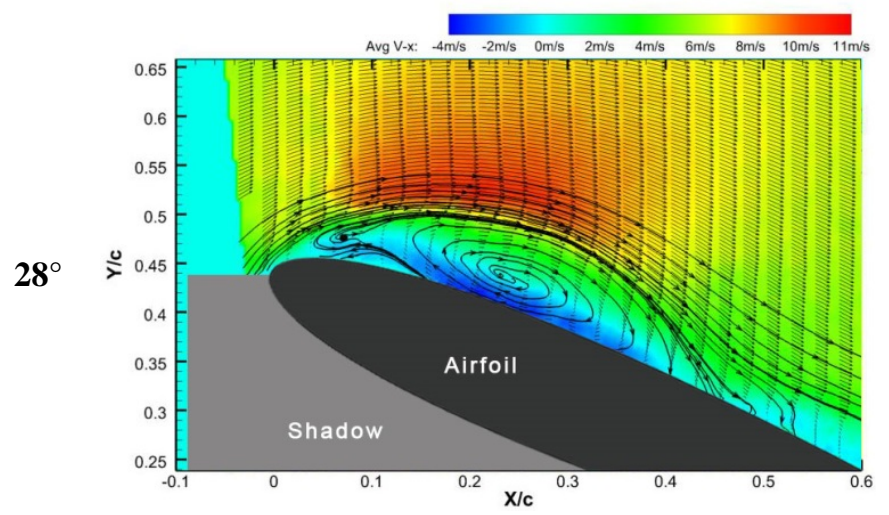

(a)

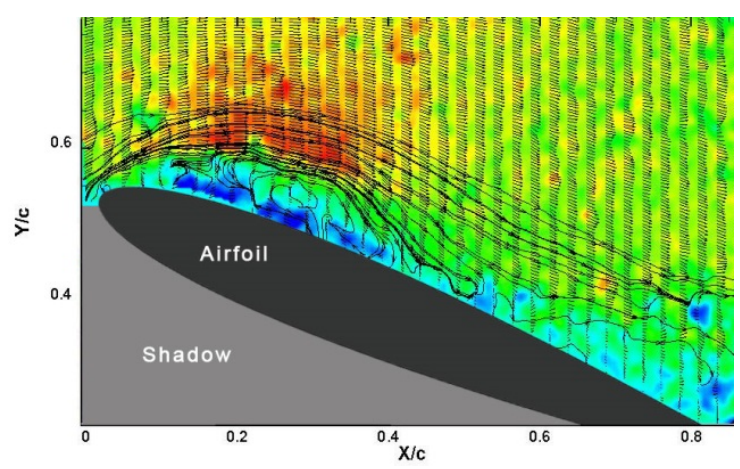

(b)

Figure 4.2 Comparison of a (a) phase averaged and an (b) instantaneous PIV calculation.

CFD results as well as the PIV analysis provides evidence that as Reynolds number increases, the effectiveness of dynamic roughness also increases. This is an interesting discovery since it has been documented and shown in this research that Reynolds number does not have a significant role in LEV formation. If this is the case, then how can Reynolds number indeed be a significant variable in whether dynamic roughness can delay LEV development? One possible answer to this question is perhaps even though the structure and LEV angle of attack onset is not affected by Reynolds number, perhaps there is a slight displacement of the initial separation point downstream during the pitching process. The angle formed by the airfoil surface, separation point, and LEV boundary also appears to be slightly smaller at higher Reynolds number. At some critical Reynolds number these factors may combine to bring the higher momentum fluid close enough to the dynamic roughness elements that it then uses this fluid to energize the fluid flow near the surface of the airfoil enough to delay the recirculation that precedes the vortex formation.

It is clear from the presented results that all methods of analysis show a trend for the ability of dynamic roughness to alter the development of the LEV on a pitching airfoil. It is obvious there are some subtle differences between the methods including recirculation region size and reattachment point. The main source of variation between numerical and experimental data is 
most likely the presence of free stream turbulence in the wind tunnel that is not modeled in the computational study as well as the absence of the inertial terms in the numerical analysis. Also, a detailed discussion was provided in Chapter 3 concerning the method of pitching simulation. This will also add to differences between the methods. It was for this reason a two-pronged approach was taken for this research. Showing a trend both computationally and experimentally provide great proof that the trend is not merely a phenomenon associated with one method or the other. 


\section{CHAPTER 5: CONCLUSIONS AND RECOMMENDATIONS}

A brief summary of what this research accomplished is as follows:

- For the first time computational and experimental analysis was able to provide proof that dynamic roughness is capable of delaying leading edge vortex (LEV) development on an airfoil undergoing a rapid pitching maneuver. The effectiveness of dynamic roughness increases as Reynolds number is increased and is robust in terms of actuation amplitude, but more sensitive to actuation frequency. Low frequency actuation did not delay the $\mathrm{LEV}$, and in some computational studies at very low Reynolds number even hastened its development.

- Computational and experimental analysis was validated, and in turn provided more evidence to the lack of Reynolds number dependence on LEV development. This research also confirmed the significant dependence LEV development has on reduced frequency, $\mathrm{k}$.

- Through computational analysis it was discovered that there is a departure from what has been observed in steady studies of dynamic roughness as a flow control device. The frequency of actuation required to delay LEV development appears to be a more precise value and not a minimum “threshold”.

- A monomial relationship between non-dimensional dynamic roughness strength and Reynolds number was developed.

- Evidence was given that both the planar and stereoscopic PIV experimental technique can be used to capture the development of the LEV, and either is robust enough to phase average even high pitching rates. The difference between stereoscopic and planar results was discussed and further insight desired to understand the contrast.

- Even though it is obvious that three-dimensional flow structures occur during LEV development, the close agreement between two-dimensional and three-dimensional simulations suggests two-dimensional simulations are sufficient to parameterize the effect of dynamic roughness. 
It is with hope that this author has at least shown further research in this area of flow control is warranted. Dynamic roughness seems to be a valid flow control device for not only steady laminar flow phenomena like leading edge laminar separation bubbles, but now also for unsteady flow conditions. Future attempts to study dynamic roughness may include:

- Development of a more definable, measurable dynamic roughness actuation system. The developed pneumatic system provides the necessary mass flow rate of air in a predictable oscillating fashion, but because there is no lubrication in the motor itself, the motor heats up quickly, which required that heat resistant tubing to be used as the air transfer conduit. This could include mechanical systems such as cam shafts, or more exotic surface disturbance mechanisms such as piezoelectric materials.

- Development of a pitching mechanism that would allow higher pitching frequencies.

- The PIV system showed it has great ability to be used as a tool to study such unsteady flow. Further investments such as a lens with a longer focal length giving the ability to focus on the leading edge region to produce results with higher resolution within the LEV itself.

- Although it has been stated a search through parameter space for conditions in which LEV development is delayed can be adequately accomplished with two-dimensional CFD, it is apparent that there are interesting structures occurring in the three-dimensional space within the LEV, especially near the dynamic roughness elements. It may prove necessary to develop simulations that incorporate turbulence models, or even Direct Numerical Simulation (DNS).

- More effort could be put for towards creating a UDF that is able to rotate the airfoil while also simulating the dynamic roughness elements. If such a UDF is developed, the inertial terms would no longer be neglected and a more accurate simulation can be obtained.

- This research may also be expanded to biological flight regimes, where it is known some biological fliers take advantage of the LEV development to enhance their lift. 


\section{REFERENCES}

1 Geissler, W. and Haselmeyer, H. Investigation of Dynamic Stall Onset. Aerospace Science and Technology, 10, 7 (2006), 590-600.

2 Carr, L. W. Progress in Analysis and Prediction of Dynamic Stall. Journal of Aircraft, 25, 1 (1988), 6-17.

3 McCroskey, W. J. Unsteady Airfoils. Annual Review of Fluid Mechanics, 14, 1 (1982), 285311.

4 El-Hak, Mohamed Gad. Flow Control: Passive, Active, and Reactive Flow Management. Cambridge University Press, 2000.

5 Gall, Peter D. A Numerical and Experimental Study of the Effects of Dynamic Roughness on Laminar Leading Edge Separation. Dissertation, West Virginia University, 2010.

6 Huebsch, W. W., Gall, P. D., Hamburg, S. D., and Rothmayer, A. P. Dynamic Roughness as a Means of Leading-Edge Separation Flow Control. Journal of Aircraft, 49, 1 (2012), 108-115.

7 Huebsch, W. W. and Rothmayer, A. P. Small-Scale Roughness Effects on Laminar Separation. Theoretical and Computational Fluid Dynamics, 17 (2003), 97-112. 10.1007/s00162-003-0094-0.

8 Huebsch, W. W. and Rothmayer, A. P. Numerical Prediction of Unsteady Vortex Shedding for Large Leading-Edge Roughness. Computers \& Fluids, 33, 3 (2004), 405-434. 
9 Huebsch, W. W. Two-Dimensional Simulation of Dynamic Surface Roughness for Aerodynamic Flow Control. Journal of Aircraft, 43, 2 (2006), 353-363.

10 Mueller, T. J. and Batill, S. M. Experimental Studies of the Laminar Separation Bubble on a Two-Dimensional Airfoil at Low Reynolds Numbers. In AIAA 13th Fluid \& Plasma Dynamics Conference ( 1980).

11 Mehta, U. B. and Lavan, Z. Starting Vortex, Separation Bubbles and Stall: A Numerical Study of Laminar Unsteady Flow Around an Airfoil. Journal of Fluid Mechanics, 67, 02 (1975), 227-256.

12 Shida, Y., Kuwahara, K., Ono, K., and Takami, H. Computation of Dynamic Stall of a NACA-0012 Airfoil. AIAA Journal, 25, 3 (1987), 408-413.

13 McCroskey, W. J. and Pucci, S. L. Viscous-Inviscid Interaction on Oscillating Airfoils in Subsonic Flow. AIAA Journal, 20, 2 (1982), 167-174.

14 Visbal, M. R. and Shang, J. S. Investigation of the Flow Structure Around a Rapidly Pitching Airfoil. AIAA Journal, 27, 8 (1989), 1044-1051.

15 Visbal, M. R. Dynamic Stall of a Constant-Rate Pitching Airfoil. Journal of Aircraft, 27, 5 (1990), 400-407.

16 Shih, C., Lourenco, L., Dommelen, L. Van, and Krothapalli, A. Unsteady Flow Past an Airfoil Pitching at a Constant Rate. AIAA Journal, 30, 5 (1992), 1153-1161.

17 Choudhuri, P. G., Knight, D. D., and Visbal, M. R. Two-Dimensional Unsteady LeadingEdge Separation on a Pitching Airfoil. AIAA Journal, 32, 4 (1994), 673-681. 
18 Rothmayer, A. P. and Bhaskaran, R. Theoretical Aspects of Stability and Transition in High Reynolds Number Dynamic Stall. In AIAA ( 1994).

19 Suito, H., Ishii, K., and Kuwahara, K. Simulation of Dynamic Stall by Multi-Directional Finite-Difference Method. In AIAA ( 1995).

20 Huebsch, W. W. and Rothmayer, A. P. Effects of Surface Ice Roughness on Dynamic Stall. Journal of Aircraft, 39, 6 (2002), 945-953.

21 Akbari, M. H. and Price, S. J. Simulation of Dynamic Stall for a NACA 0012 Airfoil Using a Vortex Method. Journal of Fluids and Structures, 17, 6 (2003), 855-874.

22 Spentzos, A., Barakos, G., Badcock, K., Richards, B., Werner, P., Schreck, S., and Raffel, M. Investigation of Three-Dimensional Dynamic Stall Using Computational Fluid Dynamics. AIAA Journal, 43, 5 (2005), 1023-1033.

23 Visbal, M. R. High-Fidelity Simulation of Transitional Flows past a Plunging Airfoil. AIAA Journal, 47, 11 (2009), 2685-2697.

24 Visbal, M. R. Numerical Investigation of Deep Dynamic Stall of a Plunging Airfoil. AIAA Journal, 49, 10 (2011), 2152-2170.

25 Martin, J. M., Empey, R. W., McCroskey, W J, and Caradonna, F. X. An Experimental Analysis of Dynamic Stall on an Oscillating Airfoil. Journal of the American Helicopter Society, 19, 1 (1974), 26-32.

26 McCroskey, W. J., Carr, L. W., and McAlister, K. W. Dynamic Stall Experiments on Oscillating Airfoils. AIAA Journal, 14, 1 (1976), 57-63. 
27 Walker, J. M., Helin, H. E., and Strickland, J. H. An Experimental Investigation of an Airfoil Undergoing Large-Amplitude Pitching Motions. AIAA Journal, 23, 8 (1985), 1141-1142.

28 Brandon, J. M. Dynamic Stall Effects and Applications to High Performance Aircraft. NASA Langley Research Center, 1991.

29 Chandrasekhara, M. S. and Ahmed, S. Laser Velocimetry Measurements of Oscillating Airfoil Dynamic Stall Flow Field. In AIAA ( 1991).

30 Acharya, M. and Metwally, H. Unsteady Pressure Field and Vorticity Production over a Pitching Airfoil. AIAA Journal, 30, 2 (1992), 403-411.

31 Ahmed, S. and Chandrasekhara, M. S. Reattachment Studies of an Oscillating Airfoil Dynamic Stall Flowfield. AIAA Journal, 32, 5 (1994), 1006-1012.

32 Shih, C., Lourenco, L. M., and Krothapalli, A. Investigation of Flow at Leading and Trailing Edges of Pitching-Up Airfoil. AIAA Journal, 33, 8 (1995), 1369-1376.

33 Bousman, W. G. Airfoil Dynamic Stall and Rotorcraft Maneuverability. NASA, 2000.

34 Schreck, S. J., Faller, W. E., and Robinson, M. C. Unsteady Separation Processes and Leading Edge Vortex Precursors: Pitch Rate and Reynolds Number Influences. Journal of Aircraft, 39, 5 (2002), 868-875.

35 Ellington, C. P., den, C. van, Willmott, A. P., and R., A. L. Leading-Edge Vortices in Insect Flight. Nature, 384, 6610 (1996), 626-630.

36 Liu, H., Ellington, C. P., Kawachi, K., den, C. van, and Willmott, A. P. A Computational Fluid Dynamic Study of Hawkmoth Hovering. Journal of Experimental Biology, 201, 4 
(1998), 461-77.

37 Birch, J. M. and Dickinson, M. H. Spanwise Flow and the Attachment of the Leading-Edge Vortex on Insect Wings. Nature, 412, 6848 (2001), 729-733.

38 Srygley, R. B. and R., A. L. Unconventional Lift-Generating Mechanisms in Free-Flying Butterflies. Nature, 420, 6916 (2002), 660-664.

39 Lu, Y., Shen, G. X., and Lai, G. J. Dual Leading-Edge Vortices on Flapping Wings. Journal of Experimental Biology, 209, 24 (2006), 5005-5016.

40 Lu, Y. and Shen, G. X. Three-Dimensional Flow Structures and Evolution of the LeadingEdge Vortices on a Flapping Wing. Journal of Experimental Biology, 211, 8 (2008), 12211230.

41 Muijres, F. T., Johansson, L. C., Barfield, R., Wolf, M., Spedding, G. R., and Hedenstrom, A. Leading-Edge Vortex Improves Lift in Slow-Flying Bats. Science, 319, 5867 (2008), 12501253.

42 Lua, K. B., Lai, K. C., Lim, T. T., and Yeo, K. S. On the Aerodynamic Characteristics of Hovering Rigid and Flexible Hawkmoth-like Wings. Experiments in Fluids, 49 (2010), 12631291. 10.1007/s00348-010-0873-5.

43 Jones, A. R. and Babinsky, H. Reynolds Number Effects on Leading Edge Vortex Development on a Waving Wing. Experiments in Fluids, 51 (2011), 197-210. 10.1007/s00348-010-1037-3.

44 Freymuth, P., Jackson, S., and Bank, W. Toward Dynamic Separation Without Dynamic Stall. 
Experiments in Fluids, 7, 3 (1988), 187-196. 10.1007/BF02332983.

45 Visbal, M. R. On the Formation and Control of the Dynamic Stall Vortex on a Pitching Airfoil. In AIAA ( 1991).

46 Karim, M. A. and Acharya, M. Suppression of Dynamic-Stall Vortices Over Pitching Airfoils by Leading-Edge Suction. AIAA Journal, 32, 8 (1994), 1647-1655.

47 Post, M. L. and Corke, T. C. Separation Control Using Plasma Actuators: Dynamic Stall Vortex Control on Oscillating Airfoil. AIAA Journal, 44, 12 (2006), 3125-3135.

48 Greenblatt, D. Active Control of Leading-Edge Dynamic Stall. International Journal of Flow Control, 2, 1 (2010), 21-38.

49 Lian, Y. Blockage Effects on the Aerodynamics of a Pitching Wing. AIAA Journal, 48, 12 (2010), 2731-2738.

50 SPECIALIST COMMITTEE ON UNCERTAINTY ANALYSIS OF $25 \mathrm{TH}$ ITTC. Uncertainty Analysis Particle Imaging Velocimetry. Recommended Procedures and Guidelines 7.5-01-03-03, International Towing Tank Conference, 2008.

51 ANSYS, INC. ANSYS FLUENT 12.0 Theory Guide. 2009. 


\section{APPENDIX A}

User Defined Function (UDF) to rotate an airfoil or domain.

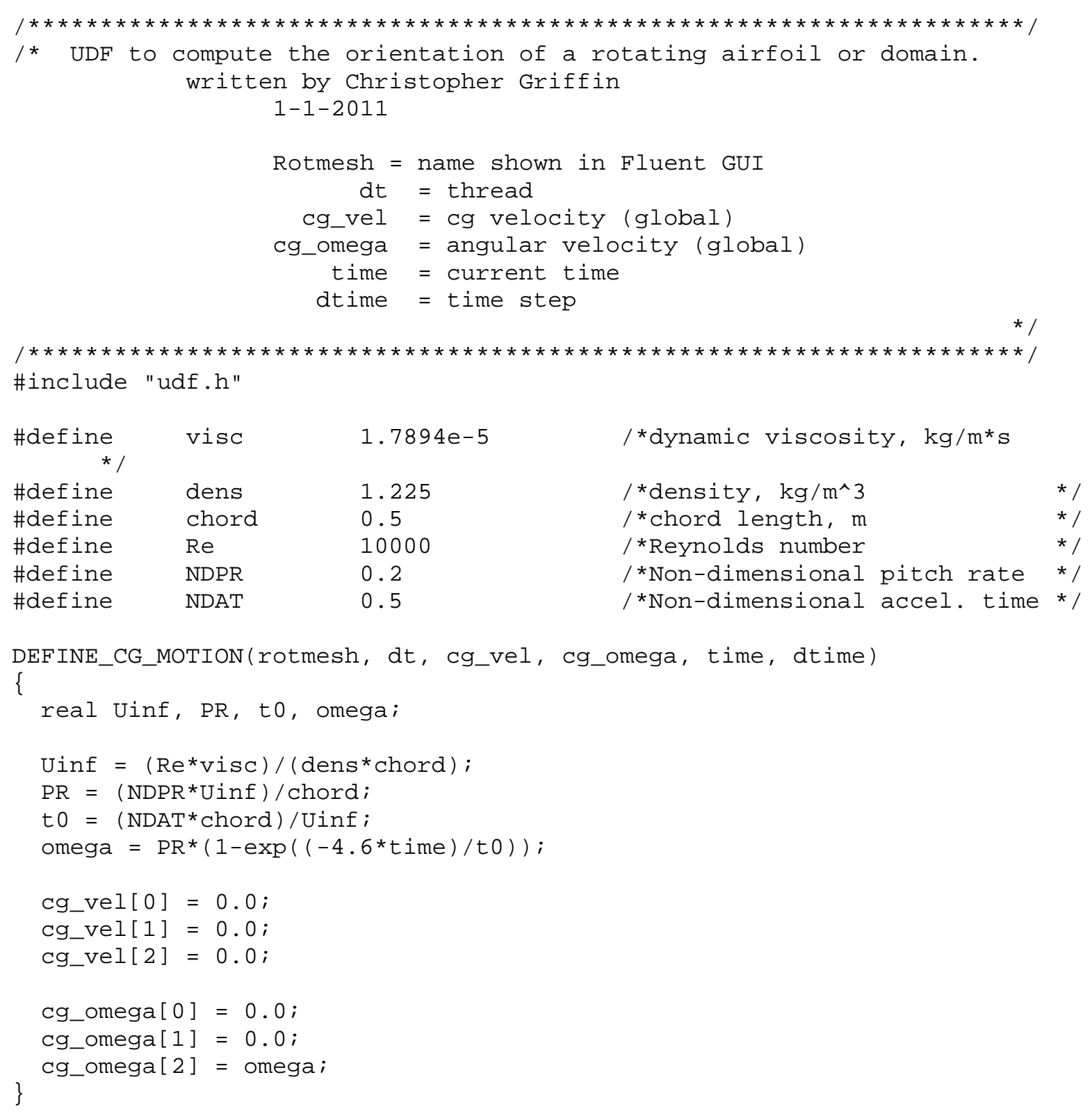




\section{APPENDIX B}

User Defined Function (UDF) to apply a variable velocity vector over the velocity inlet boundary.

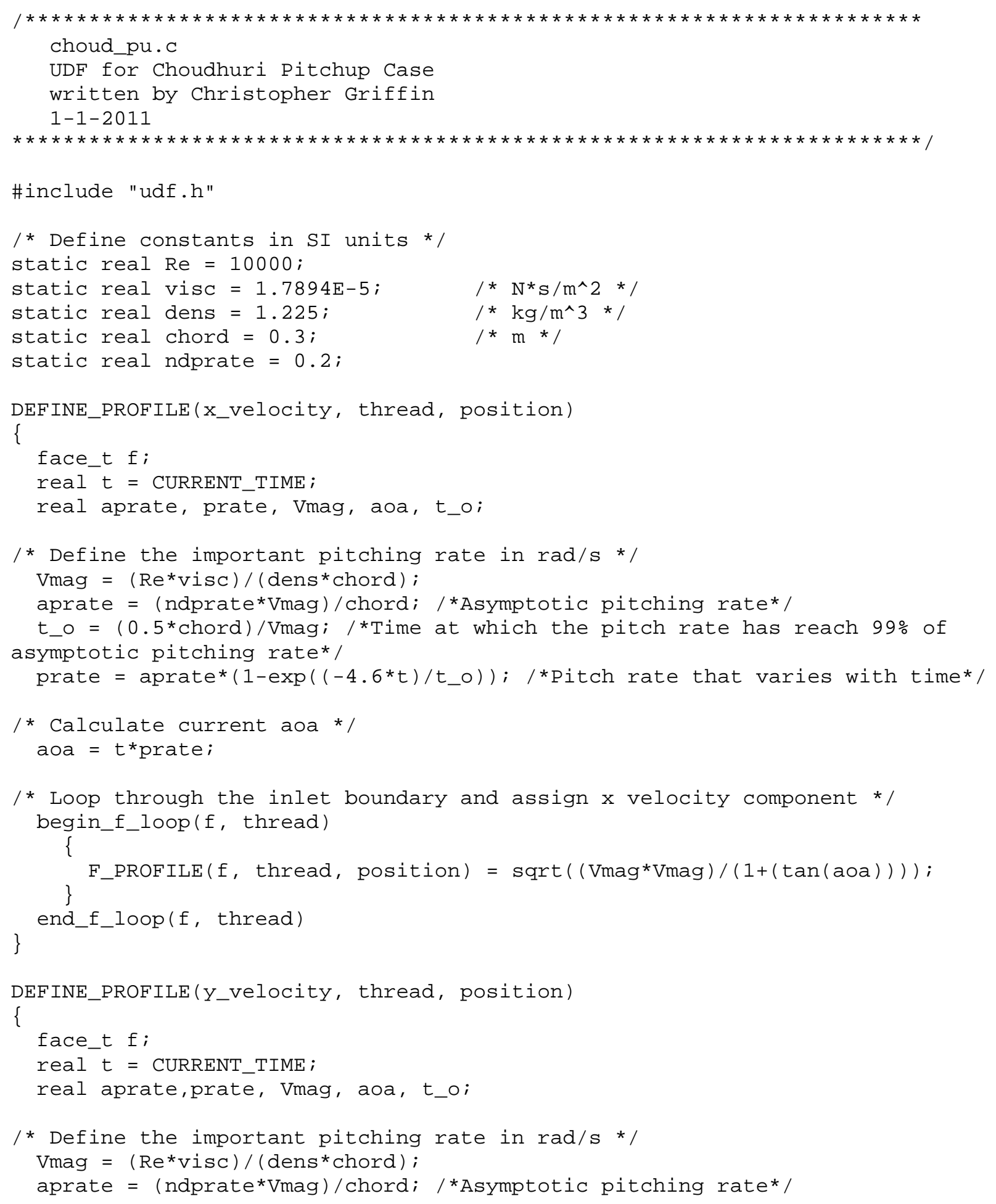




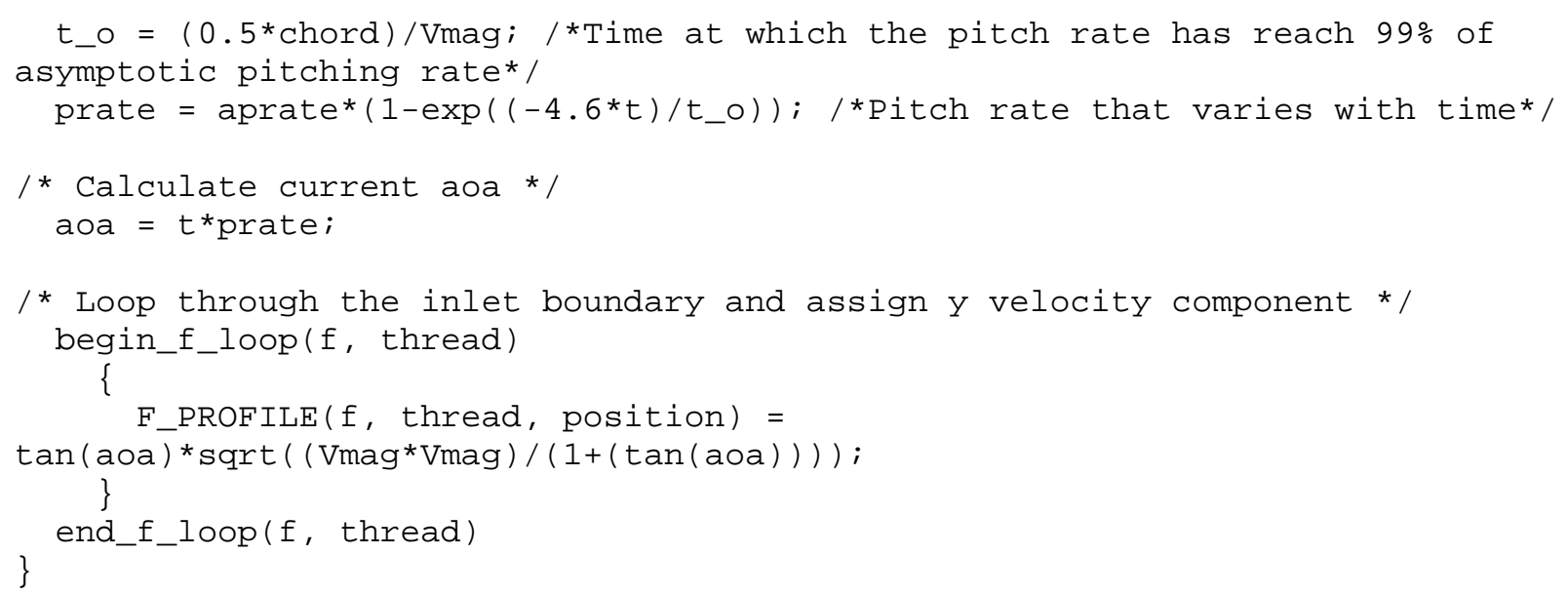




\section{APPENDIX C}

User Defined Function (UDF) to simulate dynamic roughness on airfoil surface of a twodimensional simulation. The following is code for a single dynamic roughness element. The DEFINE_GRID_MOTION function would be repeated for each necessary element.

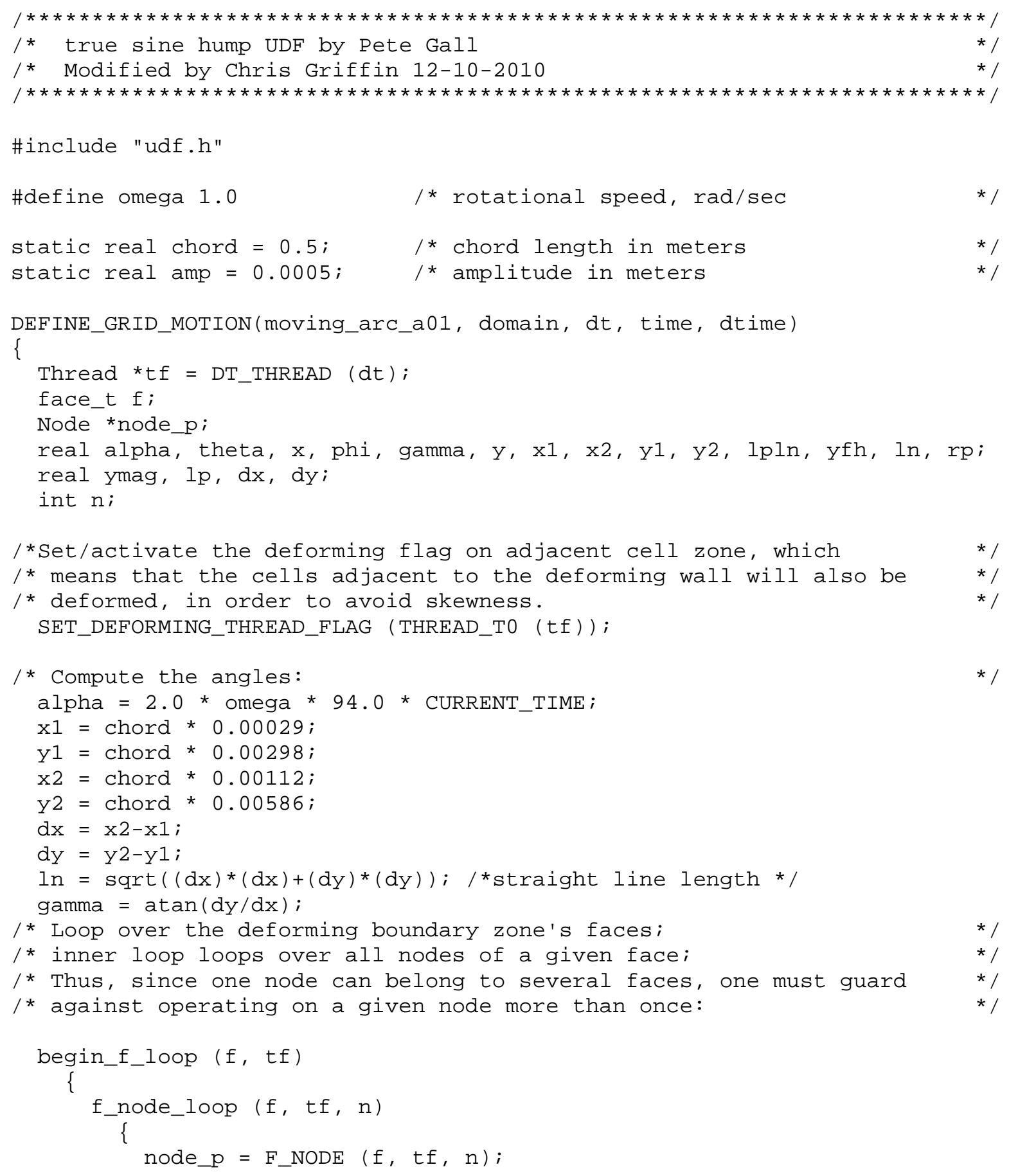




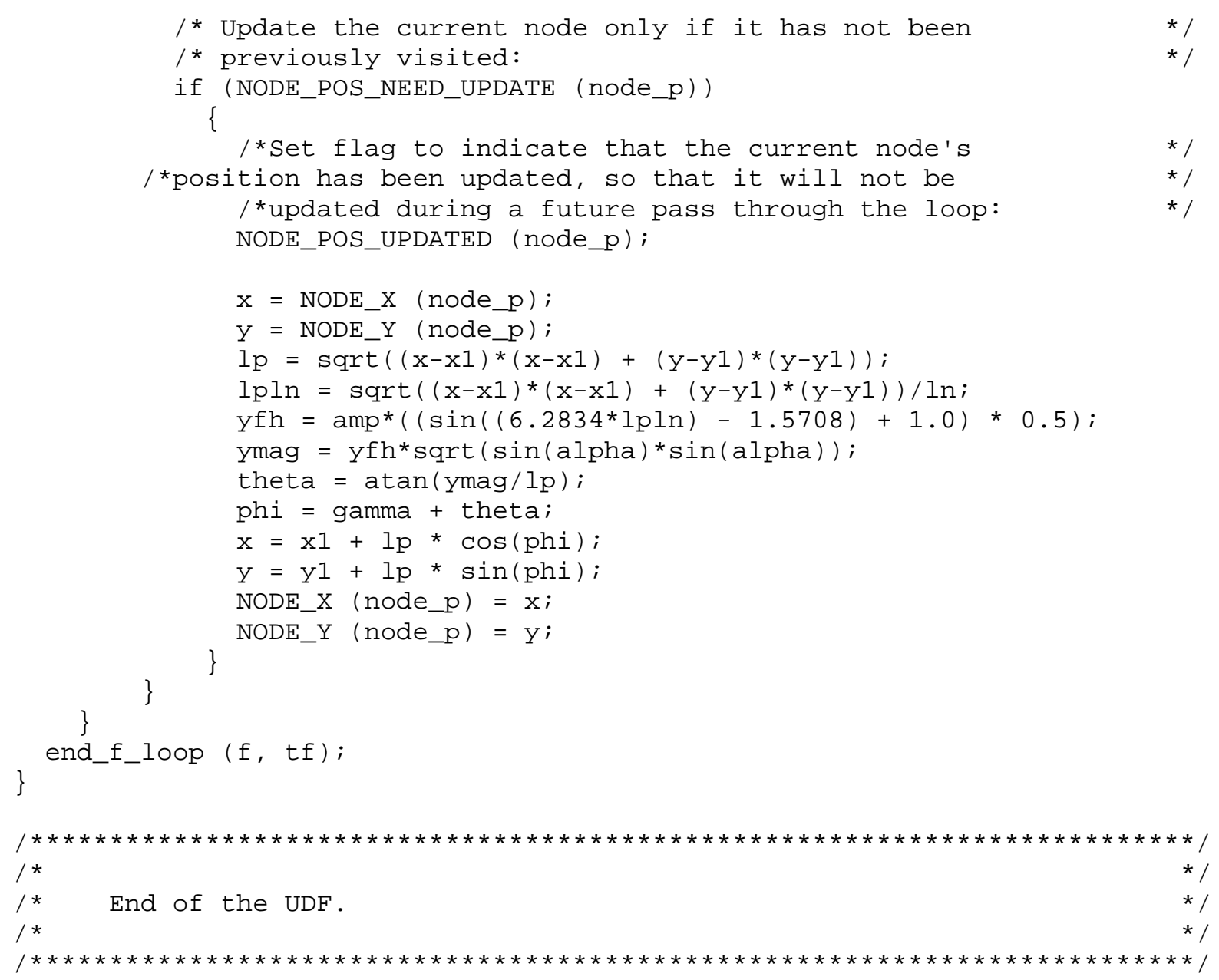




\section{APPENDIX D}

User Defined Function (UDF) to simulate dynamic roughness on airfoil surface of a twodimensional simulation. The following is code for a single dynamic roughness element. The DEFINE_GRID_MOTION function would be repeated for each necessary element.

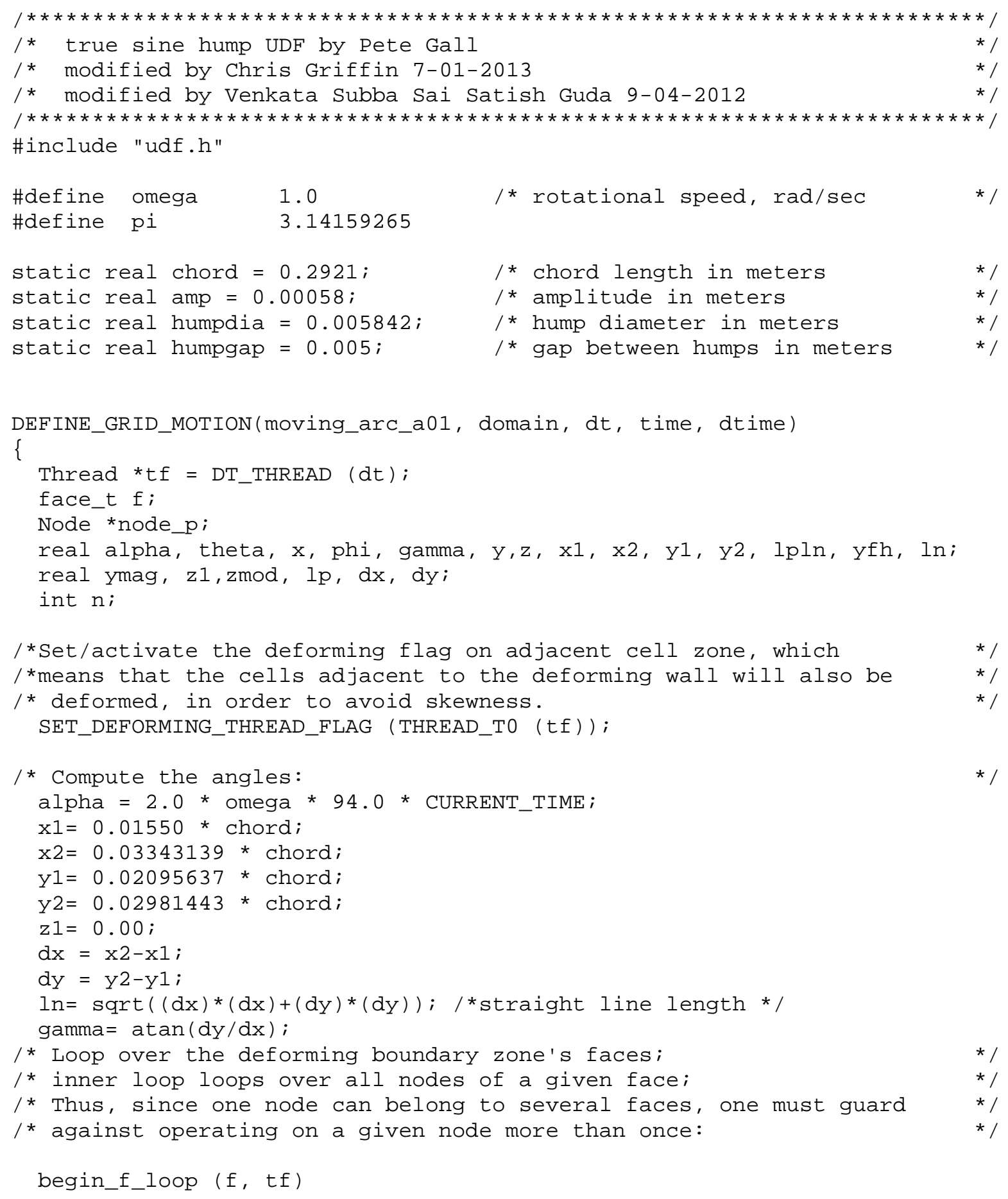




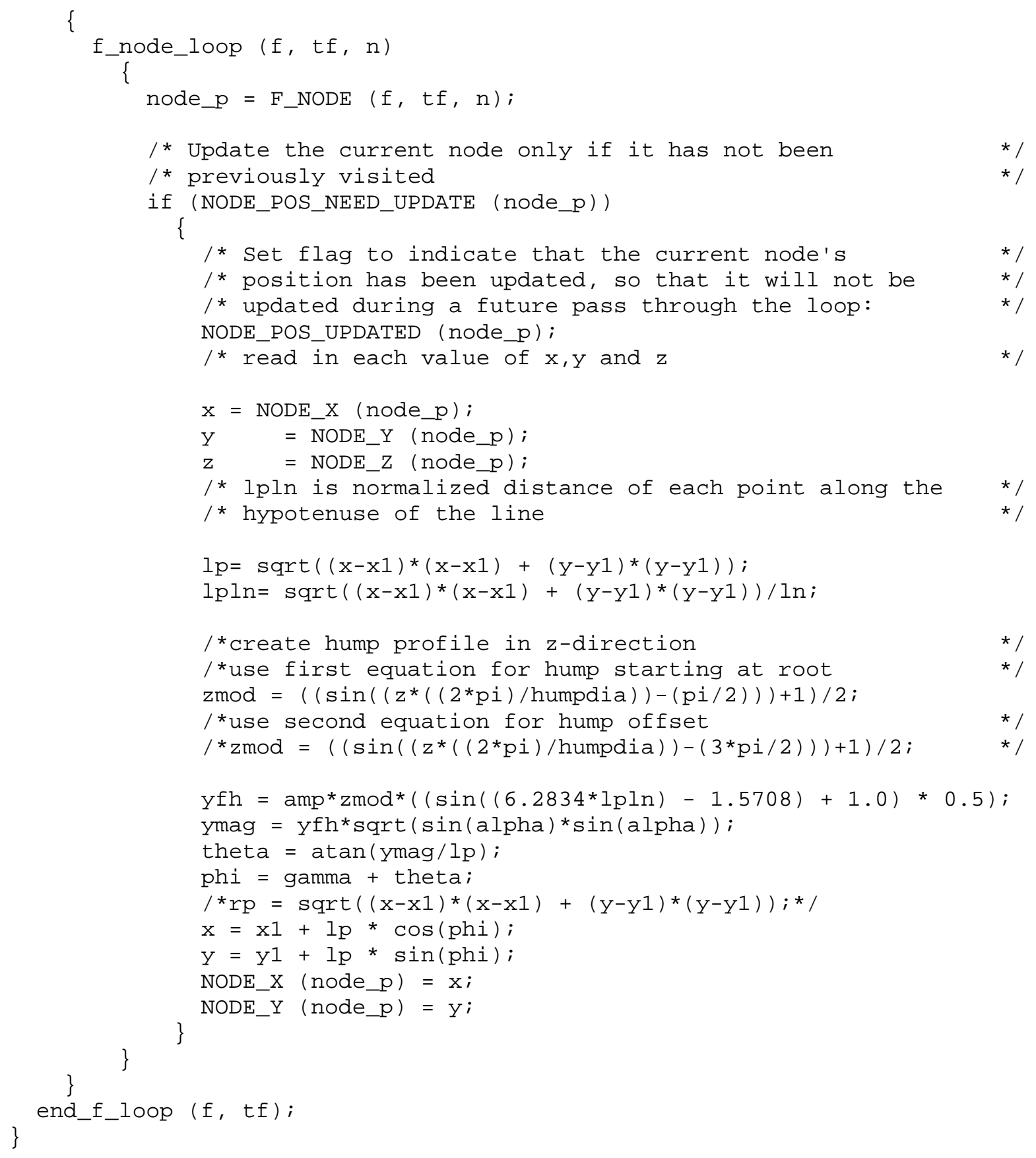

